\title{
Super-PINGU for measurement of the leptonic CP-phase with atmospheric neutrinos
}

\author{
Soebur Razzaque ${ }^{a}$ and A.Yu. Smirnov ${ }^{b, c}$ \\ ${ }^{a}$ Department of Physics, University of Johannesburg, \\ PO Box 524, Auckland Park 2006, South Africa \\ ${ }^{b}$ Max-Planck-Institute for Nuclear Physics, \\ Saupfercheckweg 1, D-69117 Heidelberg, Germany \\ ${ }^{c}$ International Centre for Theoretical Physics, \\ Strada Costiera 11, I-34100 Trieste, Italy \\ E-mail: srazzaque@uj.ac.za, smirnov@mpi-hd.mpg.de
}

ABSTRACT: We explore a possibility to measure the CP-violating phase $\delta$ using multimegaton scale ice or water Cherenkov detectors with low, $(0.2-1) \mathrm{GeV}$, energy threshold assuming that the neutrino mass hierarchy is identified. We elaborate the relevant theoretical and phenomenological aspects of this possibility. The distributions of the $\nu_{\mu}$ (track) and $\nu_{e}$ (cascade) events in the neutrino energy and zenith angle $\left(E_{\nu}-\theta_{z}\right)$ plane have been computed for different values of $\delta$. We study properties and distinguishability of the distributions before and after smearing over the neutrino energy and zenith angle. The $\mathrm{CP}$-violation effects are not washed out by smearing, and furthermore, the sensitivity to $\delta$ increases with decrease of the energy threshold. The $\nu_{e}$ events contribute to the CPsensitivity as much as the $\nu_{\mu}$ events. While sensitivity of PINGU to $\delta$ is low, we find that future possible upgrade, Super-PINGU, with few megaton effective volume at $(0.5-1) \mathrm{GeV}$ and e.g. after 4 years of exposure will be able to disentangle values of $\delta=\pi / 2, \pi, 3 \pi / 2$ from $\delta=0$ with "distinguishability" ( $\sim$ significance in $\sigma$ 's) $S_{\sigma}^{\text {tot }}=(3-8),(6-14),(3-8)$ correspondingly. Here the intervals of $S_{\sigma}^{\text {tot }}$ are due to various uncertainties of detection of the low energy events, especially the flavor identification, systematics, etc. Super-PINGU can be used simultaneously for the proton decay searches.

Keywords: CP violation, Neutrino Physics, Solar and Atmospheric Neutrinos

ARXIV EPRINT: 1406.1407 


\section{Contents}

1 Introduction 1

2 Oscillation probabilities, CP-domains 3

2.1 Oscillation amplitudes and probabilities 3

2.2 Quasi-constant density approximation 5

2.3 Numerical results 6

$\begin{array}{ll}2.4 \text { Magic lines and CP-domains } & 10\end{array}$

3 PINGU, Super-PINGU and CP 12

$\begin{array}{lll}3.1 & \text { PINGU and Super-PINGU } & 13\end{array}$

$\begin{array}{lll}3.2 & \text { Distributions of events in the neutrino energy and zenith angle plane } & 15\end{array}$

$\begin{array}{lll}3.3 & \text { CP-asymmetry and distinguishability } & 16\end{array}$

4 Distributions of the $\nu_{\mu}$ events $\quad 17$

$\begin{array}{ll}4.1 \text { The density of } \nu_{\mu} \text { events } & 17\end{array}$

4.2 Smearing 21

$\begin{array}{lll}4.3 & \text { Neutrinos and antineutrinos } & 23\end{array}$

4.4 Inverted mass hierarchy 24

5 Cascade events $\quad 24$

5.1 Density of events 24

$\begin{array}{ll}5.2 & \text { Smearing of the cascade events } 28\end{array}$

6 Sensitivity to the CP-phase 28

6.1 Total distinguishability and the energy threshold 29

$\begin{array}{lll}6.2 & \text { Correlated systematic error } & 33\end{array}$

$\begin{array}{lll}6.3 & \text { Effect of flavor misidentification } & 38\end{array}$

6.4 Sensitivity to CP-phase and its possible improvements 41

6.5 Towards realistic estimation of sensitivity 43

$\begin{array}{lll}7 & \text { Conclusions } & 44\end{array}$

\section{Introduction}

Discovery of the leptonic CP violation and measurement of the Dirac CP phase are among the main objectives in neutrino physics and, in general, in particle physics. They may have fundamental implications for theory and important consequences for phenomenology of atmospheric and accelerator neutrinos, high energy cosmic neutrinos, etc. [1-3]. 
The present experimental results have very low sensitivity to $\delta$ giving only weak indications of the preferable interval of its values. Thus, the T2K and reactor data favor the interval $\delta=(1-2) \pi$ with central value $\delta=1.5 \pi$ [4]. Analysis of the SuperKamiokande atmospheric neutrino data gives preferable range $(1.2 \pm 0.5) \pi[5]$. The global fit of all oscillation data, e.g. from [6], agrees with these results: $\delta \approx 1.39_{-0.27}^{+0.38} \pi$ at $1 \sigma$ level and no restriction appears at $3 \sigma$ level. The values around $\delta \sim \pi / 2$ are disfavored. Similar results with the best fit value $\delta=1.34 \pi(\mathrm{NH})$ have been obtained in [7] and with $\delta=251^{\circ}$ in [8].

A possibility to measure $\delta$ is generally associated with accelerator long base-line (LBL) neutrino experiments. There is certain potential to improve our knowledge of $\delta$ with further operation of T2K and NOvA [9]. Proposals of more remote experiments, which will measure $\delta$ with reasonable accuracy, include LBNE [10, 11], J-PARC — HyperKamiokande [12], ESS [13] and LBNO [14]. Further developments can be related to the low energy neutrino and muon factories, beta beams, etc., see [1-3].

Another possibility to determine $\delta$ is to use the atmospheric neutrino fluxes and large underground/underwater detectors. Sensitivity of future atmospheric neutrino studies by HyperKamiokande (HK) has been estimated in [12]: during 10 years of running with fiducial volume 0.57 Mton the HK will be able to discriminate the values of phases $\delta=40^{\circ}, 140^{\circ}, 220^{\circ}, 320^{\circ}$ at about $(1-1.5) \sigma$ CL. ICAL at INO alone will have very low sensitivity, but combined with data from T2K and NOvA, it will reduce degeneracy of parameters, and thus, increase the global sensitivity [15].

Various theoretical and phenomenological aspects of the CP-violation in atmospheric neutrinos have been explored in a number of publications before [16-29]. In particular, pattern of the neutrino oscillograms (lines of equal probabilities in the $E_{\nu}-\cos \theta_{z}$ plane) with $\mathrm{CP}$ violation has been studied in details in [22]. It was realized that structure of the oscillograms is determined to a large extent by the grid of the magic lines of three different types [22, 30-32] (solar, atmospheric and interference phase lines). Although at the probability level the effects of the CP-violation can be of order 1 , there are a number of factors which substantially reduce the effects at the level of observable events [24].

Capacities of new generation of the atmospheric neutrino detectors (PINGU, ORCA) have been explored recently [24, 33-36]. It was found [24, 34] that these detectors with $E_{\mathrm{th}} \sim 3 \mathrm{GeV}$ have good sensitivity to the neutrino mass hierarchy and the parameters of the 2-3 sector (the 2-3 mixing and mass splitting). However, the CP-violation effects turn out to be sub-leading. This helps in establishing the hierarchy without serious degeneracy with $\delta$ in contrast to the accelerator experiments, but the information on the CP-phase will be rather poor.

The goal of this paper is twofold: (i) detailed study of the CP-violation effects in atmospheric neutrinos, and (ii) tentative estimation of sensitivity to the CP-phase of future large detectors, assuming that the neutrino mass hierarchy is identified. We will show that in spite of averaging of oscillation pattern over the neutrino energy and direction, the CPviolation effects are not washed out, and furthermore, increase with lowering the energy threshold $E_{\text {th }}$. This opens up a possibility to measure $\delta$ using multi-megaton scale ice or water Cherenkov detectors with $E_{\mathrm{th}}=(0.2-0.5) \mathrm{GeV}$. We study dependence of the energy and zenith angle distributions of events produced by $\nu_{e}$ and $\nu_{\mu}$ on the CP phase. We 
estimate distinguishability of different values of $\delta$. According to the present proposal [34] PINGU will have low sensitivity to $\delta$ and only further upgrades, which we will call SuperPINGU, can measure $\delta$ with potentially competitive accuracy. We discuss requirements for such detectors. We identify problems and challenges of these $\mathrm{CP}$ measurements, and propose ways to resolve or mitigate the problems. We formulate conditions, in particular on accuracies of knowledge of external parameters and level of flavor misidentification, to achieve the goal.

The paper is organized as follows. In section 2 we summarize relevant information on the oscillation probabilities and their dependence on CP-phase. We present analytical formulas for the probabilities in quasi-constant density approximation. The grid of the magic lines will be described and we will show how the grid determines structure of oscillograms. In section 3 we consider a possible upgrade of PINGU, called Super-PINGU, which will be able to measure $\delta$ and outline a procedure of computation of numbers of events. In section 4 we compute the distributions as well as relative differences of distributions of the $\nu_{\mu}$ events in the $E_{\nu}-\cos \theta_{z}$ plane (the relative CP-differences) for different values of $\delta$. We study dependence of these distributions on $\delta$ before and after smearing over the neutrino energy and direction. In section 5 we perform similar studies of the cascade (mainly $\nu_{e}$ ) events. Section 6 contains estimations of the total sensitivity of Super-PINGU to $\delta$ and discussion of our results. We conclude in section 7 .

\section{Oscillation probabilities, CP-domains}

\subsection{Oscillation amplitudes and probabilities}

We will study the CP-violation phase $\delta$ defined in the standard parametrization of the PMNS mixing matrix, $U_{\mathrm{PMNS}}=U_{23} I_{\delta} U_{13} I_{\delta}^{*} U_{12}$, where $U_{i j}$ is the matrix of rotation in the $i j$-plane and $I_{\delta} \equiv \operatorname{diag}\left(1,1, e^{i \delta}\right)$. We consider evolution of the neutrino states $\nu_{f} \equiv$ $\left(\nu_{e}, \nu_{\mu}, \nu_{\tau}\right)^{T}$ in the propagation basis, $\nu_{\text {prop }}=\left(\nu_{e}, \tilde{\nu}_{2}, \tilde{\nu}_{3}\right)^{T}$ determined by the relation $\nu_{f}=$ $U_{23} I_{\delta} \nu_{\text {prop }}$. In this basis the CP dependence is dropped out from the evolution and appears via projection of the propagation states $\nu_{\text {prop }}$ back onto the flavor states at the production and detection. Due to this, dependence of the probabilities and numbers of events on $\delta$ is simple and explicit. Therefore the results will be presented in terms of amplitudes in this basis (see [22] for details), where the matrix of amplitudes is defined as

$$
\left\|A_{\alpha \beta}\right\|=\left(\begin{array}{ccc}
A_{e e} & A_{e \tilde{2}} & A_{e \tilde{3}} \\
\ldots & A_{\tilde{2} \tilde{2}} & A_{\tilde{2} \tilde{3}} \\
\ldots & \ldots & A_{\tilde{3} \tilde{3}}
\end{array}\right) .
$$

Here we have taken into account the equalities $A_{e \tilde{i}}=A_{\tilde{i} e}$ and $A_{\tilde{2} \tilde{3}}=A_{\tilde{3} \tilde{2}}$ valid for symmetric density profile and in absence of the fundamental $\mathrm{CP}$ and $\mathrm{T}$ violation in the propagation basis. In the low energy domain, $E \lesssim(2-3) \mathrm{GeV}$, i.e. below the 1-3 resonance, one can further decrease the number of amplitudes involved down to 3 (see [16] and comment ${ }^{1}$ ).

\footnotetext{
${ }^{1}$ The basis used in the paper [16] differs from the basis considered here by the additional 1-3 rotation on the 1-3 mixing in matter. This basis is useful for description of oscillations at low energies (in the sub-
} 
The oscillation probabilities $P_{\alpha \beta} \equiv\left|A_{\alpha \beta}\right|^{2}$ can be written as

$$
P_{\alpha \beta} \equiv P_{\alpha \beta}^{\text {ind }}+P_{\alpha \beta}^{\delta},
$$

where $P_{\alpha \beta}^{\text {ind }}$ and $P_{\alpha \beta}^{\delta}$ are the $\delta$-independent and $\delta$-dependent parts of the probability $P_{\alpha \beta}$, respectively. Notice that $P_{\alpha \beta}^{\text {ind }} \neq P_{\alpha \beta}(\delta=0)$, since $P_{\alpha \beta}^{\delta}$ contains terms which are proportional to $\cos \delta$, generally even on $\delta$, and these terms do not disappear when $\delta=0$. Then the total probability is $P_{\alpha \beta}(\delta=0)=P_{\alpha \beta}^{\text {ind }}+P_{\alpha \beta}^{0}$. The probabilities $P_{\alpha \beta}^{\text {ind }}$ equal [22]

$$
\begin{aligned}
& P_{e \mu}^{\text {ind }}=c_{23}^{2}\left|A_{e \tilde{2}}\right|^{2}+s_{23}^{2}\left|A_{e \tilde{3}}\right|^{2}, \\
& P_{\mu \mu}^{\text {ind }}=\left|c_{23}^{2} A_{\tilde{2} \tilde{2}}+s_{23}^{2} A_{\tilde{3} \tilde{3}}\right|^{2} .
\end{aligned}
$$

The amplitude $A_{\tilde{2} \tilde{3}}$ is doubly suppressed by small quantities $\Delta m_{21}^{2} / \Delta m_{31}^{2}$ and $s_{13}$ [22]. Therefore terms that are quadratic in $A_{\tilde{2} \tilde{3}}$ can be neglected in the first approximation of our analytical study. For the $\delta$-dependent parts we have then [22] $P_{e e}^{\delta}=0$,

$$
P_{e \mu}^{\delta}=\sin 2 \theta_{23} \operatorname{Re}\left[e^{i \delta} A_{e \tilde{2}}^{*} A_{e \tilde{3}}\right]=\sin 2 \theta_{23}\left|A_{e \tilde{2}} A_{e \tilde{3}}\right| \cos (\phi+\delta),
$$

where $\phi \equiv \arg \left(A_{e \tilde{2}}^{*} A_{e \tilde{3}}\right)$, and

$$
P_{\mu \mu}^{\delta}=-\sin 2 \theta_{23} \operatorname{Re}\left[A_{e \tilde{2}}^{*} A_{e \tilde{3}}\right] \cos \delta+D_{23}=-\sin 2 \theta_{23}\left|A_{e \tilde{2}} A_{e \tilde{3}}\right| \cos \phi \cos \delta+D_{23} .
$$

Here

$$
D_{23} \equiv \sin 2 \theta_{23} \cos \delta \cos 2 \theta_{23} \operatorname{Re}\left[A_{\tilde{2} \tilde{3}}^{*}\left(A_{\tilde{3} \tilde{3}}-A_{\tilde{2} \tilde{2}}\right)\right] .
$$

The term $D_{23}$ is small if the 2-3 mixing is close to the maximal one, and as we said, in addition the amplitude $A_{\tilde{2} \tilde{3}}$ is small. Let us emphasize that in $P_{\mu \mu}^{\delta}$ the phase dependence, $\cos \delta$, factors out, whereas in $P_{e \mu}^{\delta}$ it appears in combination with the oscillation phase $\phi$.

In matter with symmetric density profile one has for the inverse channels

$$
P_{\beta \alpha}=P_{\alpha \beta}(\delta \rightarrow-\delta),
$$

in particular, $P_{\mu e}^{\delta}=P_{e \mu}^{-\delta}$. For antineutrinos the probabilities have the same form as for neutrinos with substitution:

$$
\delta \rightarrow-\delta, \quad \phi_{32}^{m} \rightarrow \bar{\phi}_{32}^{m}, \quad \phi_{21}^{m} \rightarrow \bar{\phi}_{21}^{m}, \quad \theta_{i j}^{m} \rightarrow \bar{\theta}_{i j}^{m},
$$

where $\bar{\theta}_{i j}=\theta_{i j}(V \rightarrow-V)$ and $\bar{\phi}_{i j}=\phi_{i j}(V \rightarrow-V)$ are the mixing angles and phases in matter for antineutrinos, and $V$ is the matter potential. In particular,

$$
\bar{P}_{e \mu}^{\delta}=\sin 2 \theta_{23}\left|\bar{A}_{e \tilde{2}} \bar{A}_{e \tilde{3}}\right| \cos (\bar{\phi}-\delta) .
$$

GeV range) since it allows to make certain approximations which simplify description. Namely, neglecting changes of 1-3 mixing in matter with distance one can reduce 3-neutrino evolution problem to 2-neutrino evolution problem. Correspondingly all the probabilities can be expressed in terms of just three real functions $P_{2}, R$ and $I$. The main dependence on 1-3 mixing as well as on $\delta$ is explicit here. The formulas in [16] are approximate, and in general they are not valid at high energies (in multi-GeV range). Since the highest sensitivity to $\mathrm{CP}$ is at low energies these formulas give accurate description of CP-effects. 


\subsection{Quasi-constant density approximation}

One can further advance in analytical study using explicit expressions for the amplitudes in the constant (or quasi-constant) density approximation [22] (see also [37, 38] and [39]). According to this approximation, at high energies for a given trajectory in mantle one can use the mixing angles computed for the average value of the potential $V=\bar{V}\left(\theta_{z}\right)$. For low energies, where adiabaticity condition is fulfilled, the mixing angle is determined by the surface density. The oscillation phases, however, should be computed by integration over the neutrino trajectory. For core-crossing trajectories one can use the three layer model with constant densities in each layer; corrections are computed in [22].

In the case of constant density [22]

$$
\begin{aligned}
& A_{e \tilde{2}}=-i e^{i \phi_{21}^{m}} \cos \theta_{13}^{m} \sin 2 \theta_{12}^{m} \sin \phi_{21}^{m}, \\
& A_{e \tilde{3}}=-i e^{i \phi_{21}^{m}} \sin 2 \theta_{13}^{m}\left(\sin \phi_{32}^{m} e^{-i \phi_{31}^{m}}+\cos ^{2} \theta_{12}^{m} \sin \phi_{21}^{m}\right) .
\end{aligned}
$$

The half-phases equal in the high energy range (substantially larger than the 1-2 resonance, $\left.E_{\nu} \gtrsim 0.5 \mathrm{GeV}\right)$ :

$$
\phi_{32}^{m} \approx \frac{\Delta m_{31}^{2} L}{4 E_{\nu}} \sqrt{(1-\epsilon)^{2} \mp 2(1-\epsilon) \xi \cos 2 \theta_{13}+\xi^{2}} .
$$

Here $L=2 R_{E} \cos \theta_{z}$ with $R_{E}$ being the radius of the Earth,

$$
\xi \equiv \frac{2 V E_{\nu}}{\Delta m_{31}^{2}}, \quad \epsilon \equiv \sin ^{2} \theta_{12} \frac{\Delta m_{21}^{2}}{\Delta m_{31}^{2}},
$$

and the upper (lower) sign corresponds to neutrinos (antineutrinos). For two other phases we obtain

$$
\begin{aligned}
\phi_{21}^{m} & \approx \frac{\Delta m_{31}^{2} L}{8 E_{\nu}}\left[1+\xi-\epsilon\left(2 \cot ^{2} \theta_{12}-1\right)\right]-\frac{1}{2} \phi_{32}^{m}, \\
\phi_{31}^{m} & \approx \frac{\Delta m_{31}^{2} L}{8 E_{\nu}}\left[1+\xi-\epsilon\left(2 \cot ^{2} \theta_{12}-1\right)\right]+\frac{1}{2} \phi_{32}^{m},
\end{aligned}
$$

where $\phi_{32}^{m}$ is given in (2.9). In practical cases the $\epsilon$-terms can be neglected. For low energies (close to the 1-2 resonance):

$$
\phi_{21}^{m} \approx \frac{\Delta m_{21}^{2} L}{4 E_{\nu}} \sqrt{\left(\cos 2 \theta_{12} \mp \frac{2 V E_{\nu}}{\Delta m_{21}^{2}}\right)^{2}+\sin ^{2} 2 \theta_{12}} .
$$

Notice that in the energy range above the $1-2$ resonance $\cos ^{2} \theta_{12}^{m} \approx 0$ and the amplitude $A_{e \tilde{3}}(2.8)$ is reduced to the two neutrino form, which corresponds to factorization [22].

Inserting expressions for the amplitudes (2.7) and (2.8) into (2.3) we obtain

$$
P_{e \mu}^{\delta}=J_{\theta} \sin \phi_{21}^{m}\left[\sin \phi_{32}^{m} \cos \left(\delta-\phi_{31}^{m}\right)+\cos ^{2} \theta_{12}^{m} \sin \phi_{21}^{m} \cos \delta\right],
$$

where

$$
J_{\theta} \equiv \sin 2 \theta_{23} \sin 2 \theta_{12}^{m} \sin 2 \theta_{13}^{m} \cos \theta_{13}^{m}
$$


is the mixing angles factor of the Jarlskog invariant in matter. Using relation $\phi_{31}^{m}=\phi_{32}^{m}+\phi_{21}^{m}$ we obtain from $(2.13)$

$$
P_{e \mu}^{\delta} \approx J_{\theta} \sin \phi_{21}^{m}\left[\frac{1}{2} \sin 2 \phi_{32}^{m} \cos \left(\delta-\phi_{21}^{m}\right)+\sin ^{2} \phi_{32}^{m} \sin \left(\delta-\phi_{21}^{m}\right)+\cos ^{2} \theta_{12}^{m} \sin \phi_{21}^{m} \cos \delta\right] .
$$

Similarly, neglecting $D_{23}$ we find for $P_{\mu \mu}^{\delta}$

$$
P_{\mu \mu}^{\delta}=-\cos \delta J_{\theta} \sin \phi_{21}^{m}\left[\sin \phi_{32}^{m} \cos \phi_{31}^{m}+\cos ^{2} \theta_{12}^{m} \sin \phi_{21}^{m}\right],
$$

or excluding $\phi_{31}^{m}: \phi_{31}^{m}=\phi_{32}^{m}+\phi_{21}^{m}$

$$
P_{\mu \mu}^{\delta}=-\cos \delta J_{\theta} \sin \phi_{21}^{m}\left[\frac{1}{2} \sin 2 \phi_{32}^{m} \cos \phi_{21}^{m}-\sin ^{2} \phi_{32}^{m} \sin \phi_{21}^{m}+\cos ^{2} \theta_{12}^{m} \sin \phi_{21}^{m}\right],
$$

where $\delta$ dependence factors out.

For antineutrinos we have the same expressions (2.15) and (2.17) with substitution (2.5) and $J_{\theta} \rightarrow \bar{J}_{\theta}=J_{\theta}\left(\theta_{i j} \rightarrow \bar{\theta}_{i j}\right)$.

We will use the analytic expressions (2.15) and (2.17) and the corresponding expressions for antineutrinos for interpretation of numerical results.

\subsection{Numerical results}

We have computed the probabilities $P_{\alpha \beta}=P_{\alpha \beta}\left(E_{\nu}, \theta_{z}\right)$ by performing numerical integration of the evolution equation for the complete $3 \nu$-system. We used the PREM density profile of the Earth [40] and the values of the neutrino parameters $\Delta m_{32}^{2}=2.35 \cdot 10^{-3} \mathrm{eV}^{2}$, $\Delta m_{21}^{2}=7.6 \cdot 10^{-5} \mathrm{eV}^{2}, \sin ^{2} \theta_{23}=0.42, \sin ^{2} \theta_{12}=0.312$ and $\sin ^{2} \theta_{13}=0.025$, which are close to the current best fit values [6]. We assume the normal neutrino mass hierarchy in the most part of the paper.

In figure 1 we show the oscillation probabilities $\nu_{e} \rightarrow \nu_{\mu}$ and $\nu_{\mu} \rightarrow \nu_{\mu}$ as functions of the neutrino energy for different values of CP-phase and zenith angles. In the low energy range where sensitivity to $\delta$ is high and consider $P_{e \mu}$. In figure 1 the resonantly enhanced probability due to the 1-2 mixing and mass splitting is modulated by fast oscillations driven by the 1-3 mass and mixing. The 1-2 resonance energy in the mantle is at $E_{12}^{R} \approx 0.12 \mathrm{GeV}$. For core crossing trajectories (upper panels) the parametric effects distort the dependence of probability on energy.

The key feature which opens up a possibility to measure $\delta$ is the presence of systematic shift of the oscillatory curves (probabilities) at low energies, $\lesssim 2 \mathrm{GeV}$, with increase of the phase. The shift occurs in the same way in wide energy interval $E_{\nu}=(0.2-2) \mathrm{GeV}$, and essentially for all trajectories which cross the mantle only. This systematic shift can be understood using analytical expressions for the probabilities. Averaging $P_{e \mu}^{\delta}(2.15)$ over fast oscillations driven by the 1-3 splitting we find

$$
\left\langle P_{e \mu}^{\delta}\right\rangle=\frac{J_{\theta}}{2}\left[\cos \delta \cos 2 \theta_{12}^{m} \sin ^{2} \phi_{21}^{m}+\frac{1}{2} \sin \delta \sin 2 \phi_{21}^{m}\right] .
$$



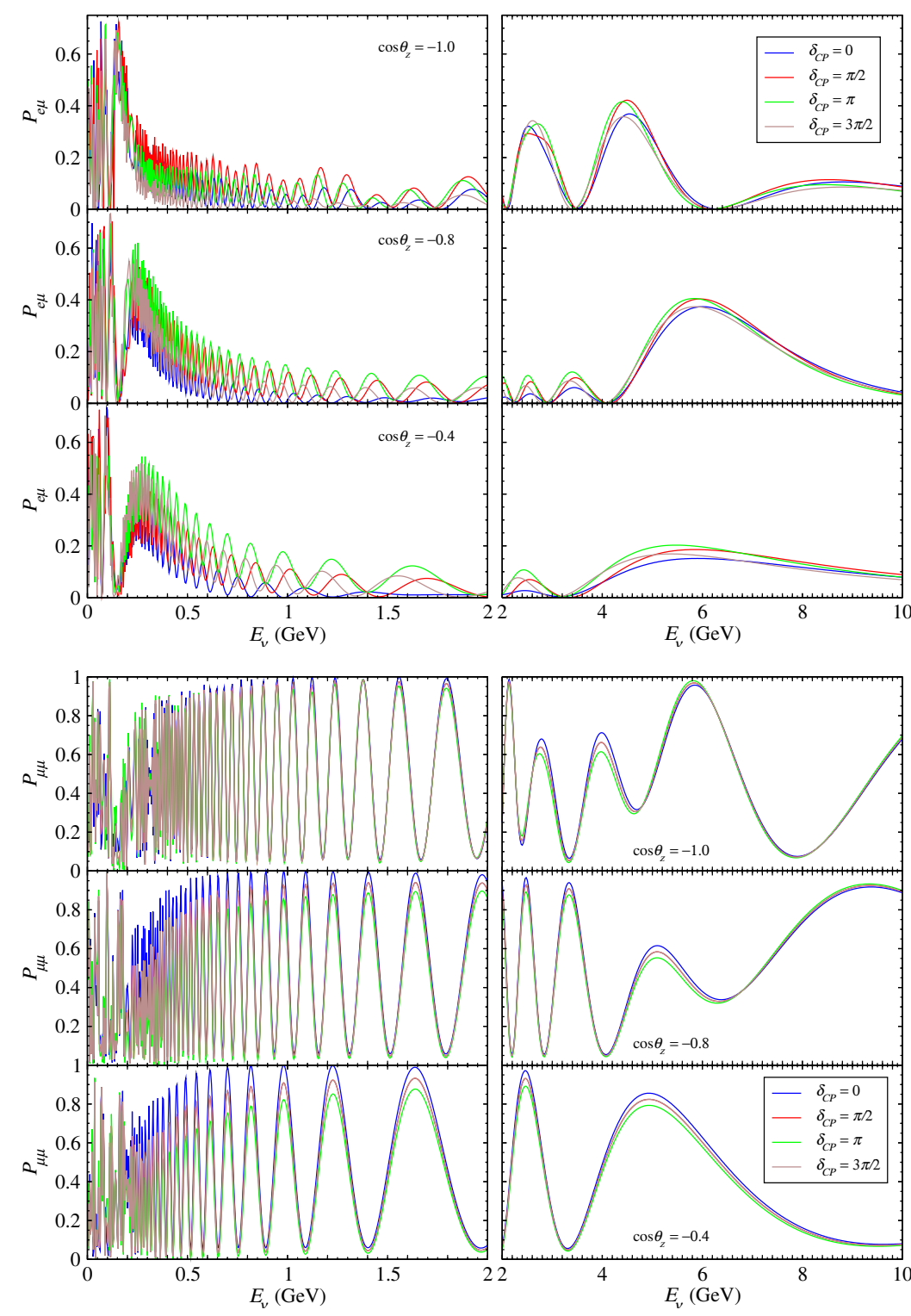

Figure 1. Probabilities of the $\nu_{e} \rightarrow \nu_{\mu}$ (top panels) and $\nu_{\mu} \rightarrow \nu_{\mu}$ (bottom panels) oscillations as functions of the neutrino energy for different values of $\delta$ and the zenith angle. The probability $P_{\mu \mu}$ is the same for $\delta=\pi / 2$ and $3 \pi / 2$ in the bottom panels. Normal mass hierarchy is assumed and the neutrino parameters from the global fits are used (see main text).

The first term does not change the sign with $\phi_{21}^{m}$, whereas the second one does. Notice that above the $1-2$ resonance $\cos 2 \theta_{12}^{m} \approx-1$, and so

$$
\left\langle P_{e \mu}^{\delta}\right\rangle \approx \frac{J_{\theta}}{2}\left[-\cos \delta \sin ^{2} \phi_{21}^{m}+\frac{1}{2} \sin \delta \sin 2 \phi_{21}^{m}\right] .
$$

The difference of probabilities for a given value $\delta$ and $\delta=0$ equals:

$$
\left\langle P_{e \mu}^{\delta}\right\rangle-\left\langle P_{e \mu}^{0}\right\rangle=\frac{J_{\theta}}{2}\left[(1-\cos \delta) \sin ^{2} \phi_{21}^{m}+\frac{1}{2} \sin \delta \sin 2 \phi_{21}^{m}\right] .
$$



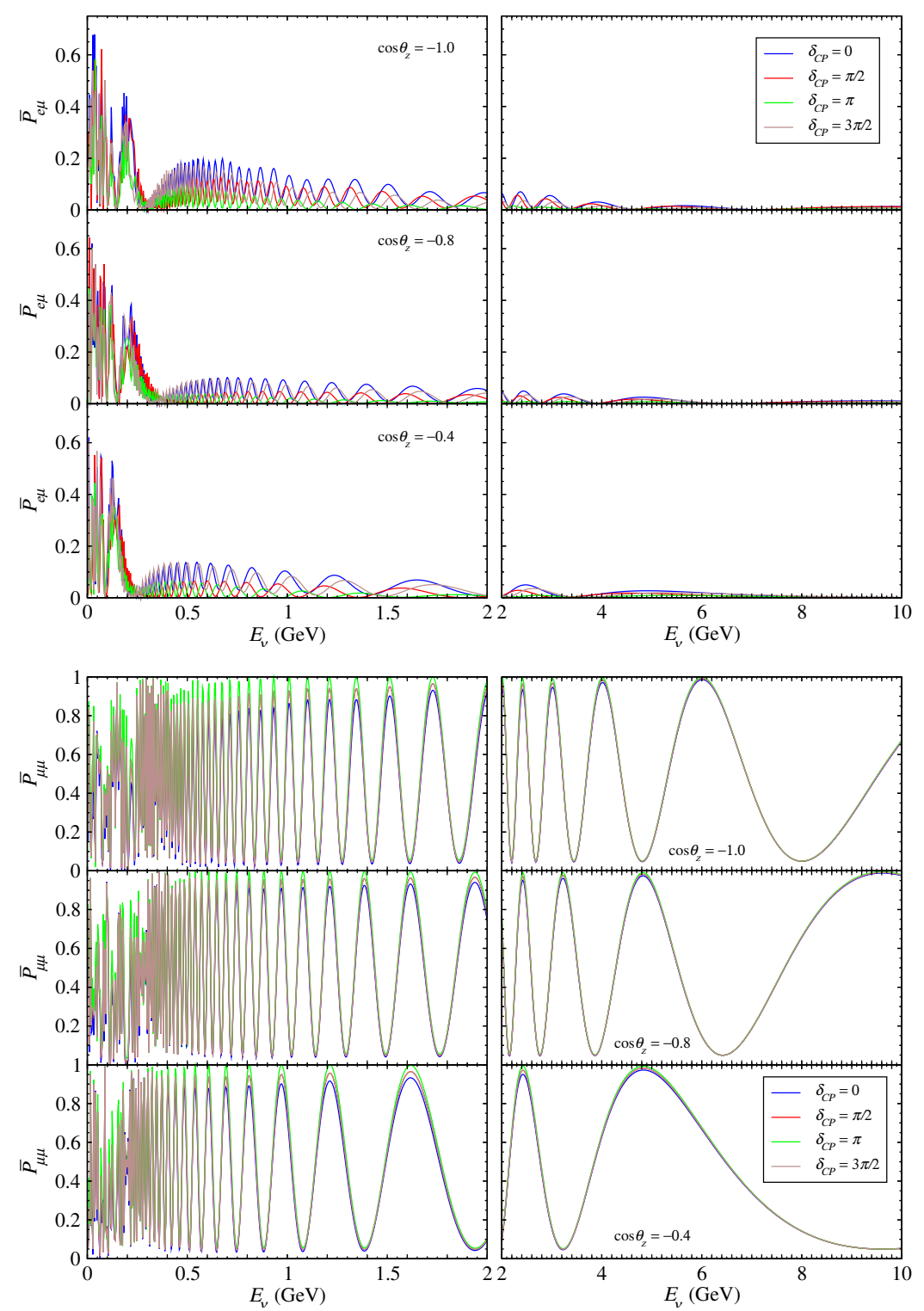

Figure 2. Same as figure 1, but for antineutrinos.

The first term is positive for all $\phi_{21}^{m}$ and $\delta$, and it is this term that produces a systematic shift of the probabilities.

For the values of $\delta$-phase shown in figure 1 we obtain from (2.18)

$$
\begin{aligned}
\left\langle P_{e \mu}^{0}\right\rangle & =-\left\langle P_{e \mu}^{\pi}\right\rangle=\frac{J_{\theta}}{2} \cos 2 \theta_{12}^{m} \sin ^{2} \phi_{21}^{m}, \\
\left\langle P_{e \mu}^{\pi / 2}\right\rangle & =-\left\langle P_{e \mu}^{3 \pi / 2}\right\rangle=\frac{J_{\theta}}{4} \sin ^{2} 2 \phi_{21}^{m} .
\end{aligned}
$$

These equations show that $\left\langle P_{e \mu}^{0}\right\rangle$ is the smallest one. The probability increases with $\delta$ and reaches maximum at $\delta=\pi$. For the trajectory with $\cos \theta_{z}=-0.4$, the oscillation phase equals $\phi_{21}^{m} \approx \pi / 2$. That leads to $\left\langle P_{e \mu}^{\pi / 2}\right\rangle=\left\langle P_{e \mu}^{3 \pi / 2}\right\rangle=0$, and consequently, to 
equal total probabilities. For $\cos \theta_{z}=-0.8$ the phase equals $\phi_{21}^{m}=1.42 \pi$ which gives different values of probability: $\left\langle P_{e \mu}^{\pi / 2}\right\rangle=-\left\langle P_{e \mu}^{3 \pi / 2}\right\rangle=0.24 J_{\theta} / 4=0.06 J_{\theta}$, and furthermore $\left\langle P_{e \mu}^{\pi}\right\rangle=-\left\langle P_{e \mu}^{0}\right\rangle=0.94 J_{\theta} / 2=0.47 J_{\theta}$. These results are in agreement with plots shown in figure 1.

Although there is certain phase shift with change of $\delta$, the sizes of energy intervals where the difference $P\left(\delta_{1}\right)-P\left(\delta_{2}\right)$ has positive and negative signs are strongly different. One sign dominates, and therefore there is no averaging over energy. Maximal relative upward shift of the probability curves compared to the $\delta=0$ curve is around $(0.4-1) \mathrm{GeV}$. For the core-crossing trajectories $\left(\cos \theta_{z}<-0.83\right)$ due to the parametric effects the transition probability first increases with increase of $\delta$, it reaches maximum at $\delta \sim \pi / 2$ and then decreases.

The $\nu_{\mu}-\nu_{\mu}$ probability, $P_{\mu \mu}^{\delta}(2.17)$, averaged over the 1-3 oscillations equals

$$
\left\langle P_{\mu \mu}^{\delta}\right\rangle=-\frac{J_{\theta}}{2} \cos \delta \sin ^{2} \phi_{21}^{m} \cos 2 \theta_{12}^{m},
$$

where the $D_{23}$ term is neglected. Notice immediately that the CP-effect in the $\nu_{\mu}-\nu_{\mu}$ channel has an opposite sign with respect to that in the $\nu_{e}-\nu_{\mu}$ channel (2.18). Therefore the presence of both $\nu_{e}$ and $\nu_{\mu}$ original fluxes weakens the total CP-effect, and consequently, the sensitivity to $\delta$ which is unavoidable. We will call this the flavor suppression.

According to (2.22) dependence of the $\nu_{\mu}-\nu_{\mu}$ probability on $\delta$ factors out and therefore turns out to be very simple. The maximal effect is for $\delta=0$,

$$
\left\langle P_{\mu \mu}^{0}\right\rangle=-\left\langle P_{\mu \mu}^{\pi}\right\rangle \approx \frac{J_{\theta}}{2} \sin ^{2} \phi_{21}^{m},
$$

and $\left\langle P_{\mu \mu}^{\pi / 2}\right\rangle=\left\langle P_{\mu \mu}^{3 \pi / 2}\right\rangle=0$, so that the total probabilities are equal for $\pi / 2$ and $3 \pi / 2$ which in perfect agreement with result of figure 1 .

The difference of probabilities for a given value of $\delta$ and zero phase equals

$$
\left\langle P_{\mu \mu}^{\delta}\right\rangle-\left\langle P_{\mu \mu}^{0}\right\rangle=\frac{J_{\theta}}{2}(1-\cos \delta) \sin ^{2} \phi_{21}^{m} \cos 2 \theta_{12}^{m} \approx-\frac{J_{\theta}}{2}(1-\cos \delta) \sin ^{2} \phi_{21}^{m} .
$$

Only CP-even contribution is present.

The probabilities in antineutrino channels are shown in figure 2. Their dependencies on $E_{\nu}$ and $\cos \theta_{z}$ can be immediately understood from our analytical treatment. According to (2.5) the averaged probabilities equal

$$
\begin{aligned}
& \left\langle\bar{P}_{e \mu}^{\delta}\right\rangle=\frac{\bar{J}_{\theta}}{2}\left[\cos \delta \cos 2 \bar{\theta}_{12}^{m} \sin ^{2} \bar{\phi}_{21}^{m}-\frac{1}{2} \sin \delta \sin 2 \bar{\phi}_{21}^{m}\right], \\
& \left\langle\bar{P}_{\mu \mu}^{\delta}\right\rangle=-\frac{\bar{J}_{\theta}}{2} \cos \delta \sin ^{2} \bar{\phi}_{21}^{m} \cos 2 \bar{\theta}_{12}^{m} .
\end{aligned}
$$

For energies far above the 1-2 resonance, the expressions are further simplified since $\cos 2 \bar{\theta}_{12}^{m} \approx 1$ (recall, for neutrinos $\cos 2 \theta_{12}^{m} \approx-1$ ):

$$
\left\langle\bar{P}_{e \mu}^{\delta}\right\rangle=\frac{\bar{J}_{\theta}}{2}\left[\cos \delta \sin ^{2} \bar{\phi}_{21}^{m}-\frac{1}{2} \sin \delta \sin 2 \bar{\phi}_{21}^{m}\right], \quad\left\langle\bar{P}_{\mu \mu}^{\delta}\right\rangle=-\frac{\bar{J}_{\theta}}{2} \cos \delta \sin ^{2} \bar{\phi}_{21}^{m} .
$$


Comparing this with (2.20) and (2.22) we find that for antineutrinos the probabilities have opposite sign with respect to the probabilities for neutrinos. Indeed, according to figure 2 for mantle trajectories the biggest amplitude $\bar{P}_{e \mu}$ is for $\delta=0$ and the smallest one is for $\delta=\pi$ which is opposite to the $P_{e \mu}$ case. This means that summation of signals from neutrinos and antineutrinos reduces the effect of CP-phase, and consequently, the sensitivity to this phase. This $C$-suppression can be reduced if $\nu$ and $\bar{\nu}$ signals are separated at least partially (see section 6.3).

As follows from figure 2 for the mantle crossing trajectories, only the largest CP effect on $\bar{P}_{e \mu}$ is in the range $E_{\nu}=(0.4-0.7) \mathrm{GeV}$ where maximal values equal $\bar{P}_{e \mu} \approx 0.1$ and 0.15 for $\cos \theta_{z}=-0.8$ and -0.4 correspondingly. These numbers are about 2 times smaller than for neutrinos. The reason is that, in the case of $\mathrm{NH}$ for neutrinos both $\theta_{12}^{m}$ and $\theta_{13}^{m}$ are enhanced in matter whereas for antineutrinos both $\bar{\theta}_{12}^{m}$ and $\bar{\theta}_{13}^{m}$ are suppressed. The antineutrino probabilities decrease with increase of energy above $0.8 \mathrm{GeV}$. This, as well as smaller $\bar{\nu}$ cross-sections suppresses number of $\bar{\nu}$ events and therefore reduces cancellation of the CP-effect.

Similar consideration can be performed for the $\nu_{\mu}-\nu_{\mu}$ channel for which $\left\langle\bar{P}_{\mu \mu}^{\delta}\right\rangle=$ $-\bar{J}_{\theta} 2 \cos \delta \sin ^{2} \bar{\phi}_{21}^{m}$. Notice that in vacuum $\left\langle\bar{P}_{\mu \mu}^{\delta}\right\rangle=\left\langle P_{\mu \mu}^{\delta}\right\rangle$, i.e. the probability is even function of $\delta$. In the matter dominated region we have $\left\langle\bar{P}_{\mu \mu}^{\delta}\right\rangle \approx-\left\langle P_{\mu \mu}^{\delta}\right\rangle$ due to change of sign of the potential. The differences of the antineutrino probabilities for a given $\delta$ and $\delta=0$ equals at $\cos 2 \theta_{12}^{m} \approx 1$

$$
\begin{aligned}
\left\langle\bar{P}_{e \mu}^{\delta}\right\rangle-\left\langle\bar{P}_{e \mu}^{0}\right\rangle & =-\frac{\bar{J}_{\theta}}{2}\left[(1-\cos \delta) \sin ^{2} \bar{\phi}_{21}^{m}+\frac{1}{2} \sin \delta \sin 2 \bar{\phi}_{21}^{m}\right], \\
\left\langle\bar{P}_{\mu \mu}^{\delta}\right\rangle-\left\langle\bar{P}_{\mu \mu}^{0}\right\rangle & =\frac{\bar{J}_{\theta}}{2}(1-\cos \delta) \sin ^{2} \bar{\phi}_{21}^{m} .
\end{aligned}
$$

They also have an opposite sign with respect to the differences for neutrinos (2.20) and (2.23), and equal up to change of mixing angles and phases in matter.

\subsection{Magic lines and CP-domains}

In what follows we will study differences of probabilities as well as distributions of events in the $E_{\nu}-\cos \theta_{z}$ plane for different values of $\delta$. The patterns of distributions are determined to a large extent by the grid of the magic lines [22, 30-32]. The lines fix the borders of the CP-domains - the regions in the $E_{\nu}-\cos \theta_{z}$ plane of the same sign of the CP-difference.

Let us summarize relevant information about properties of the magic lines. Recall that the magic lines are defined as the lines in the $E_{\nu}-\theta_{z}$ plane along which the oscillation probabilities do not depend on phase $\delta$ in the so called factorization (quasi $2 \nu$ ) approximation [22]. Correspondingly, the CP-differences vanish along these lines.

(i) The solar magic lines are determined by the condition

$$
\phi_{S}=\phi_{21}^{m}=n \pi, \quad n=1,2,3, \ldots
$$

where in neutrino channels $\phi_{S}$ is given by the expression (2.12) for $\phi_{21}^{m}$ valid in $3 \nu$ framework below 1-3 resonance but extended to all the energies. For antineutrinos 
in the NH case $\bar{\phi}_{S}=\bar{\phi}_{21}^{m}$ everywhere. Along these lines $\left|A_{e \tilde{2}}\right|=0$ below the 1-3 resonance. That would be the line of zero solar amplitude in the $2 \nu$ approximation. The minimum of $\nu_{e}-\nu_{\mu}$ probability at $\sim 0.15 \mathrm{GeV}$ for $\cos \theta_{z}=-0.4$ in figure 1 corresponds to the first magic line with $\phi_{S}=\phi_{21}^{m}=\pi$. The minimum at $0.17 \mathrm{GeV}$ for $\cos \theta_{z}=-0.8$ (figure 1) is on the second magic line with $\phi_{21}^{m}=2 \pi$.

Notice that the energy of minimal level splitting (maximal oscillation length) is given by $E_{12}^{R} / \cos ^{2} 2 \theta_{12} \approx 0.7 \mathrm{GeV}$ which is much bigger than $E_{12}^{R}=0.12 \mathrm{GeV}$ due to large 12 mixing. So, below $0.7 \mathrm{GeV}$ the splitting increases and correspondingly the oscillation length decreases. Therefore the same phase can be obtained for smaller $\left|\cos \theta_{z}\right|$, and consequently, the solar magic lines bend toward smaller $\left|\cos \theta_{z}\right|$. At energies much above the 1-2 resonance these lines do not depend on energy and are situated at

$$
\cos \theta_{z}=-0.60,-0.86,-0.97,
$$

for $\phi_{21}^{m}=\pi, 2 \pi$, and $3 \pi$ correspondingly.

(ii) The atmospheric magic lines are determined by the equality

$$
\phi_{A}=\phi_{23}^{m}=n \pi, \quad n=1,2,3, \ldots
$$

Along these lines $\left|A_{e \tilde{3}}\right| \approx 0$. It would vanish exactly in the $2 \nu$ approximation, when $\cos ^{2} \theta_{12}^{m} \approx 0$ that is far above the 1-2 resonance. Zeros of the $\nu_{e}-\nu_{\mu}$ probability at $E_{\nu} \geq 2 \mathrm{GeV}$ (see figure 1) which do not depend on $\delta$ are situated on the atmospheric magic lines. E.g., for $\cos \theta_{z}=-0.4$ these points are at $E_{\nu}=2 \mathrm{GeV}$ and $E_{\nu}=3.2 \mathrm{GeV}$. For $\cos \theta_{z}=-0.8$, zeros are at $E_{\nu}=2.3,2.9,4.1 \mathrm{GeV}$. For $P_{\mu \mu}$ the solar and atmospheric magic lines coincide with those for $P_{e \mu}$ in the limit $D_{23}=0$.

The magic lines determined by (2.29) and (2.31) do not coincide with lines where $\left|A_{e \tilde{2}}\right|=0$ and $\left|A_{e \tilde{3}}\right|=0$ in the $3 \nu$ framework. But they play the role of asymptotics of the true lines where dependence of probabilities on $\delta$ disappears. The latter interpolate between different magic lines.

(iii) The interference phase lines are important for distinguishing different values of the CP-phase: a given value $\delta$ and a different value $\delta_{0}$. Along these lines $P_{\alpha \beta}^{\delta}-P_{\alpha \beta}^{\delta_{0}}=0$. According to (2.3) for $P_{e \mu}$ the condition reads

$$
\cos (\phi+\delta)=\cos \left(\phi+\delta_{0}\right)
$$

where $\phi \approx-\phi_{31}$ and the latter is the vacuum oscillation phase. This condition corresponds to intersection of probability curves for different values of phases $\delta$ and $\delta_{0}$ in figure 1. For $\delta_{0}=0$ the condition can be written as $\phi_{31}+\delta=-\phi_{31}$ or

$$
\phi_{31}=\frac{\Delta m_{31}^{2} L}{4 E_{\nu}}=-\frac{\delta}{2}+n \pi .
$$

For the inverse channel, $\nu_{\mu} \rightarrow \nu_{e}$, the sign of $\delta$ should be changed. According to (2.4) dependencies of the $\nu_{\mu} \rightarrow \nu_{\mu}$ probability on $\phi$ and $\delta$ factor out in the approximation 
$D_{23}=0$, and the corresponding interference phase line is determined by the condition $\cos \phi=0$, or

$$
\phi \approx \phi_{31}=\frac{\pi}{2}+n \pi
$$

The condition can be written as

$$
E_{\nu}=-A(\phi) \cos \theta_{z}=-\frac{R_{E} \Delta m_{31}^{2}}{2 \phi(\delta)} \cos \theta_{z}
$$

where $R_{E}$ is the Earth radius and in general $\phi(\delta)$ should lead to the vanishing CPdifference of probabilities.

The exact value of interference phase $\phi$ does not coincide with $-\phi_{31}$. In the constant density approximation $\phi$ equals the phase of the expression in brackets of $A_{e \tilde{3}}(2.8)$ :

$$
\tan \phi=-\frac{\sin \phi_{32}^{m} \sin \phi_{31}^{m}}{\cos \phi_{31}^{m} \sin \phi_{32}^{m}+\cos ^{2} \theta_{12}^{m} \sin \phi_{21}^{m}}
$$

Notice that $\phi$ would be equal $-\phi_{31}^{m}$, if $\cos \phi_{31}^{m}=0$. The latter is satisfied for high energies $E \gg E_{12}^{R}$, where $\cos ^{2} \theta_{12}^{m} \approx 0$. However, if $\phi_{31}^{m} \approx \pi / 2$ we can not neglect the second term in the denominator of (2.34). Notice that in the limit $\cos ^{2} \theta_{12}^{m}=0$ we obtain from (2.16)

$$
P_{\mu \mu}^{\delta}=-\cos \delta J_{\theta} \sin \phi_{21}^{m} \sin \phi_{32}^{m} \cos \phi_{31}^{m},
$$

where one can see immediately all three "magic" conditions.

Notice that magic lines could be introduced immediately in the $3 \nu$ framework as the lines along which $P_{\alpha \beta}^{\delta}-P_{\alpha \beta}^{\delta_{0}}=0$. In this case they would, indeed, determine the borders of domains with different sign of the CP-difference of the probabilities. We use the original definitions of magic lines to match with previous discussions. Still as we said before, the solar, atmospheric and interference lines nearly coincide with the exact lines of zero CPdifferences in certain energy intervals or in asymptotics. The corresponding phases are related as

$$
\phi_{21}^{m} \approx\left\{\begin{array}{ll}
\phi_{S}^{m}, & E \ll E_{31}^{R} \\
\phi_{A}^{0}, & E \geq E_{31}^{R}
\end{array}, \quad \phi_{31}^{m} \approx\left\{\begin{array}{ll}
\phi_{A}^{0}, & E \ll E_{31}^{R} \\
\phi_{S}, & E \gg E_{31}^{R}
\end{array}, \quad \phi_{32}^{m} \approx \phi_{A}^{m},\right.\right.
$$

where $\phi_{A}^{0}$ is the phase in vacuum. So, the true lines of zero difference of probabilities interpolate between the magic lines (see [22] for details).

\section{PINGU, Super-PINGU and CP}

The key conclusion of the previous section is that integration over the neutrino energy and direction does not suppress the CP effect significantly. Furthermore, for all trajectories which cross the mantle of the Earth only, the CP violation effect is similar: it has the same sign and the same change with $\delta$. Effect is different for the core crossing trajectories, $\cos \theta_{z}<-0.83$. So, there could be partial cancellation due to smearing over the zenith angle. Another important feature is that the relative $\mathrm{CP}$ effect at the probability level increases with decrease of energy. In this connection we will explore a possibility to measure 
$\delta$ using multi-megaton scale neutrino detectors with low energy threshold. As it was already realized in [24], sensitivity of PINGU to $\delta$ is low. So, we will consider future possible upgrades of PINGU. We will also quantify capacity of PINGU to obtain information about $\delta$. For definiteness we will speak about PINGU for which more information is available. Similar upgrades can be considered for ORCA detector [35].

\subsection{PINGU and Super-PINGU}

We calculate event rates for the proposed PINGU detector and for possible future PINGU upgrade which we call Super-PINGU. The PINGU detector [34] will have 40 strings additional to the DeepCore strings with 60 digital optical modules (DOMs) at $5 \mathrm{~m}$ spacing in each string. A compact array like PINGU could detect neutrinos with energies as low as (1-3) GeV. Strict criteria allow over $90 \%$ efficiency of event reconstruction for all 3 flavors [34]. We parametrize the PINGU effective mass as

$$
\rho V_{\text {eff }, \mu}\left(E_{\nu}\right)=3.0\left[\log \left(E_{\nu} / \mathrm{GeV}\right)\right]^{0.61} \mathrm{Mt}
$$

and

$$
\rho V_{\text {eff }, e}\left(E_{\nu}\right)=3.1\left[\log \left(E_{\nu} / \mathrm{GeV}\right)\right]^{0.60} \mathrm{Mt},
$$

respectively for $\nu_{\mu}$ and $\nu_{e}$. Here $V_{\text {eff }, \alpha}$ is the effective volume and $\rho$ is the density of the ice. These parametrizations well represent simulated volumes [34] from $\gtrsim 1 \mathrm{GeV}$ up to $25 \mathrm{GeV}$. We will use an accuracy of the energy and angle reconstruction for PINGU from [34].

Along with the PINGU proposal the idea has been discussed to construct "ultimate" multi-megaton-scale detector MICA with a threshold about $10 \mathrm{MeV}$ allowing to detect the solar and supernova neutrinos [41]. Clearly reducing the threshold by more that 2 orders of magnitude is very challenging. In this connection we would like to consider a kind of intermediate step - the detector with an effective energy threshold about (0.1-0.2) GeV, i.e. one order of magnitude below the threshold in the present PINGU proposal. For this, a denser array of DOMs is required which will lead to increase of the effective volume of a detector at low energies. For definiteness we will take the effective volume which corresponds to the PINGU detector simulations with a total of 126 strings and 60 DOMs per string each [42]. The effective mass can be parameterized as

$$
\rho V_{\text {eff }}\left(E_{\nu}\right)=2.6\left[\log \left(E_{\nu} / \mathrm{GeV}\right)+1\right]^{1.32} \mathrm{Mt},
$$

for both $\nu_{\mu}$ and $\nu_{e}$ events. We call this version Super-PINGU. According to (3.1) and (3.3) the effective mass, $\rho_{\text {eff }} V_{\text {eff }}$, in the range (1-2) GeV equals 0.7 Mton for PINGU and $\sim 2.8$ Mton for Super-PINGU, i.e. 4 times larger. For the bin below $1 \mathrm{GeV}$ the corresponding numbers are 0.3 and 2.2 Mton ( 7 times larger). This can be compared with MICA, which may have 220 strings and 140 DOMs per string. We will extrapolate to lower energies some PINGU characteristics from the proposal [34].

Going to further upgrade has double effect:

- increase of the effective volume, especially in the low energy bins, and

- improvements of reconstruction of the neutrino energy and direction as well as the flavor identification of events for all energies. 
Super-PINGU will have three times denser DOM array than PINGU. Therefore it will collect about 3 times more photons from the same event (with the same neutrino energy). Recall that, in PINGU the average distance between DOMs is smaller than the photon scattering length $(50 \mathrm{~m})$.

We describe uncertainties of reconstruction of the neutrino energy and direction by smearing functions

$$
G_{E}\left(E_{\nu}^{r}, E_{\nu}\right), \quad G_{\theta}\left(\theta_{z}^{r}, \theta_{z}\right)
$$

where $E_{\nu}$ and $\theta_{z}\left(E_{\nu}^{r}\right.$ and $\left.\theta_{z}^{r}\right)$ are the true (reconstructed) energy and zenith angle of the neutrinos. For PINGU we use $G_{E}$ and $G_{\theta}$ from [34] determined down to energies $\sim 1 \mathrm{GeV}$. The distributions are normalized in such a way that

$$
\int d E_{\nu} d \theta_{z} G_{E}\left(E_{\nu}^{r}, E_{\nu}\right) G_{\theta}\left(\theta_{z}^{r}, \theta_{z}\right)=1 .
$$

Notice that PINGU distributions have longer tails than the Gaussian functions.

Characteristics of the Super-PINGU reconstruction are expected to be better. We estimate parameters of $G_{E}$ and $G_{\theta}$ for Super-PINGU using the DeepCore resolutions and the simulated PINGU resolutions [43-45] in the following way. For a given event the number of photons collected is proportional to the density of DOMs, that is $N_{\mathrm{DOM}}$ for fixed total volume of the detector. Therefore the relative statistical error in determination of characteristics is proportional to $1 / \sqrt{N_{\mathrm{DOM}}}$, so we can assume that

$$
\sigma_{\theta} \propto \frac{1}{\sqrt{N_{\mathrm{DOM}}}}, \quad \sigma_{E} \propto \frac{1}{\sqrt{N_{\mathrm{DOM}}}} .
$$

Estimations of resolutions of the DeepCore and PINGU confirm (3.4). Indeed, DeepCore has about $N_{\mathrm{DOM}}^{\mathrm{DC}}=530$ DOMs, while PINGU $(40$ strings with 60 DOMs per string) will have $N_{\mathrm{DOM}}^{\mathrm{PINGU}}=2400 \mathrm{DOMs}$ (also with higher quantum efficiency), that is, $N_{\mathrm{DOM}}^{\mathrm{PINGU}} / N_{\mathrm{DOM}}^{\mathrm{DC}}=4.5$. Since the density of DOMs in PINGU is about 4.5 times larger, amount of light detected from the same event will be about 4.5 times larger. According to [43-45] and [34] for the $\nu_{\mu}$ events the ratio of resolutions (median errors)

$$
\frac{\sigma_{\theta}^{\mathrm{PINGU}}}{\sigma_{\theta}^{\mathrm{DC}}} \approx 0.5 .
$$

The ratio equals 0.66 at $E_{\nu}=5 \mathrm{GeV}$, however estimation of DC parameters become not very reliable at low energies. For the $\nu_{e}$ events the improvement is even better: the ratio of median errors (3.5) is $(0.38-0.43)$ in the interval $E_{\nu}=(10-20) \mathrm{GeV}$ and it becomes 0.6 at $5 \mathrm{GeV}$.

For neutrino energy reconstruction (median energy resolution) of the $\nu_{\mu}$ events we have

$$
\frac{\sigma_{E}^{\mathrm{PINGU}}}{\sigma_{E}^{\mathrm{DC}}} \approx 0.58-0.61
$$

in the interval $E_{\nu}=(10-20) \mathrm{GeV}$. It decreases down to 0.52 at $E_{\nu}=5 \mathrm{GeV}$. Similar improvement is expected for the $\nu_{e}$ events. 
The Super-PINGU will have 3 times larger number (and therefore density) of DOMs, than PINGU. Therefore according to (3.4) the resolutions will be further improved by factor $1 / \sqrt{3} \approx 0.58$. So for Super-PINGU we use the resolution functions from figure 7 and 8 of [34], scaling their widths as

$$
\sigma_{\theta}^{\text {SuperPINGU }}=\frac{1}{\sqrt{3}} \sigma_{\theta}^{\mathrm{PINGU}}, \quad \sigma_{E}^{\mathrm{SuperPINGU}}=\frac{1}{\sqrt{3}} \sigma_{E}^{\mathrm{PINGU}} .
$$

We extrapolate these functions down to $E_{\nu}=0.5 \mathrm{GeV}$ and for simplicity neglect possible dependences of the factors in eq. (3.7) on energy. (Notice that according to [34] the median value of angle is very similar for cascades and tracks.)

This estimation of improvement can be considered as conservative. Indeed, the DeepCore characteristics have been obtained after stringent kinematical cuts which allows one to select a sample of high quality events. That reduces efficiency of reconstruction (fraction of reconstructed events) down to (10-20)\%, whereas PINGU characteristics have been obtained with (60-70)\% efficiency. With stronger cuts in PINGU the reconstruction characteristics could be even better. Also, developments of electronics may lead to further improvements. Clearly, configuration of Super-PINGU should be optimized taking into account also the cost of construction. For large density of strings the issue of the ice stability may become important. One can reduce number of strings by increasing number of DOMs per string (decreasing vertical spacing). Since typical size of an event is about $100 \mathrm{~m}$, for distances between strings $(17 \mathrm{~m})$ the total number of DOMs in the unit volume matters and geometry plays only secondary role. Another option is to consider underwater detector, i.e., an upgrade of ORCA.

\subsection{Distributions of events in the neutrino energy and zenith angle plane}

To evaluate sensitivity of Super-PINGU to $\delta$ we will compute the $\left(E_{\nu}-\cos \theta_{z}\right)$ distributions of events of different types and explore their dependence on $\delta$. The numbers of events $N_{\alpha}$, produced by neutrinos $\nu_{\alpha}(\alpha=e, \mu)$ with energies and zenith angles in small bins $\Delta\left(E_{\nu}\right)$ and $\Delta\left(\cos \theta_{z}\right)$ marked by subscript $j i$ equal

$$
N_{i j, \alpha}=2 \pi N_{A} \rho T \int_{\Delta_{i} \cos \theta_{z}} d \cos \theta_{z} \int_{\Delta_{j} E_{\nu}} d E_{\nu} V_{\mathrm{eff}, \alpha}\left(E_{\nu}\right) d_{\alpha}\left(E_{\nu}, \theta_{z}\right) .
$$

Here $T$ is the exposure time, $N_{A}$ is the Avogadro's number. The density of events of type $\alpha, d_{\alpha}$, (the number of events per unit time per target nucleon) is given by

$$
d_{\alpha}\left(E_{\nu}, \theta_{z}\right)=d_{\alpha}^{\nu}+d_{\alpha}^{\bar{\nu}}=\left[\sigma_{\alpha} \Phi_{\alpha}+\bar{\sigma}_{\alpha} \bar{\Phi}_{\alpha}\right],
$$

where $\Phi_{\alpha}$ and $\bar{\Phi}_{\alpha}$ are the fluxes of neutrinos and antineutrinos at the detector which produce events of the type $\alpha$, and $\sigma_{\alpha}$ and $\bar{\sigma}_{\alpha}$ are the corresponding cross-sections. In turn, the fluxes at the detector equal

$$
\Phi_{\alpha}=\Phi_{\mu}^{0} P_{\mu \alpha}+\Phi_{e}^{0} P_{e \alpha},
$$

$\Phi_{\mu}^{0}=\Phi_{\mu}^{0}\left(E_{\nu}, \theta_{z}\right)$ and $\Phi_{e}^{0}=\Phi_{e}^{0}\left(E_{\nu}, \theta_{z}\right)$ are the original muon and electron neutrino fluxes at the production. 
With decrease of energy, resonance processes (pion production) and quasi-elastic processes will contribute, and the latter dominates below $1 \mathrm{GeV}$. In our estimations we use the total neutrino-nucleon cross-sections down to $(0.2-0.3) \mathrm{GeV}$ as they are parametrized in [34], We assume that different contributing processes would produce visible effect at the detector with the same efficiency. For antineutrinos there is no data below $1 \mathrm{GeV}$ and we use extrapolation given in [34]. Clearly in future these computations should be refined.

We use the atmospheric neutrino fluxes, $\Phi_{\mu}^{0}$ and $\Phi_{e}^{0}$ (and corresponding fluxes of antineutrinos) from refs. [46, 47]. At low energies the geomagnetic effects become important which break azimuthal symmetry.

After smearing in the $\left(E_{\nu}^{r}-\cos \theta_{z}^{r}\right)$ plane, we obtained the unbinned distribution of events as

$$
N_{\alpha}\left(E^{r}, \cos \theta^{r}\right)=2 \pi N_{A} T \rho \int d \cos \theta_{z} \int d E_{\nu} G_{E}\left(E_{\nu}^{r}, E_{\nu}\right) G_{\theta}\left(\theta_{z}^{r}, \theta_{z}\right) V_{\mathrm{eff}}\left(E_{\nu}\right) d_{\alpha}\left(E_{\nu}, \cos \theta_{z}\right),
$$

$\alpha=e, \mu$, and then binned them according to

$$
N_{i j, \alpha}=\int_{\Delta_{i}\left(\cos \theta_{z}^{r}\right)} d \cos \theta_{z}^{r} \int_{\Delta_{j}\left(E_{\nu}^{r}\right)} d E_{\nu}^{r} N_{\alpha}\left(E^{r}, \cos \theta_{z}^{r}\right),
$$

with $\Delta\left(E_{\nu}^{r}\right)=0.5 \mathrm{GeV}$ and $\Delta\left(\cos \theta_{z}^{r}\right)=0.025$. Again, we can split number of events onto $\delta$ - dependent and $\delta$-independent parts: $N_{i j}(\delta)=N_{i j}^{\text {ind }}+N_{i j}^{\delta}$.

\subsection{CP-asymmetry and distinguishability}

As in [24], we will employ the distinguishability $S_{\sigma}$ as a quick estimator of sensitivity of measurements. For a given type of events (we omit the index $\alpha$ ) and each $i j$-bin we define the relative $\mathrm{CP}$-difference as

$$
S_{i j}(f)=\frac{N_{i j}^{\delta}-N_{i j}^{0}}{\sigma_{i j}},
$$

where $N_{i j}^{\delta}$ and $N_{i j}^{0}$ are the numbers of events computed for a given value of $\delta$ and for $\delta=0$ correspondingly, and

$$
\sigma_{i j}^{2}=N_{i j}(\delta=0)+\left[f N_{i j}(\delta=0)\right]^{2}=N_{i j}^{\text {ind }}+N_{i j}^{0}+f^{2}\left(N_{i j}^{\text {ind }}+N_{i j}^{0}\right)^{2}
$$

is the total "error" in the $i j$-bin. If $N_{i j}(\delta=0)$ is interpreted as a result of measurement, the first term in the equation above would correspond to the statistical error and the second one to the uncorrelated systematic errors. As in [24] we assume that the latter is proportional to the number of events: $f N_{i j}(\delta=0)$. In general $f$ is a function of neutrino energy and zenith angle. The uncorrelated errors could be due to local impurities (dust) in the ice, uncontrolled efficiency of individual DOMs, uncertainties in neutrino fluxes and cross-sections (on top of overal normalization and tilt uncertainties). The level of these uncertainties is not known. So, only what we can do is to explore how sensitivity changes depending on the level of uncertainties. This allows us to conclude about tolerable level of $f$.

Notice that, since here contribution from the systematic error is proportional to $\left(N_{i j}^{0}\right)^{2}$, for the same $f$ the role of this error decreases with decreasing size of the bin. Since here we 
use 2 times larger both in $E_{\nu}$ and $\cos \theta_{z}$ bins after smearing than in [24], to keep the same level of systematic errors with respect to statistical error we need to use 2 times smaller $f$. For illustration we will take values $f=2.5 \%$ and $5 \%$ which correspond to the cases when uncorrelated systematic error smaller and comparable with the statistical error. Notice that $f=2.5 \%$ gives the closest approximation of our results to the results of PINGU simulations [34] in the case of sensitivity to the neutrino mass hierarchy.

If $N_{i j}^{\delta}$ is considered as the fit value, the moduli $\left|S_{i j}\right|$ would give the standard deviation and so the statistical significance. However, in contrast to real situation the "measured" value $N_{i j}^{0}$ does not fluctuate. Therefore we will not interpret it as number of sigmas, but just use $|S|$ as independent characterization - the distinguishability.

Considering the effect in each bin as an independent measurement (which is possible after smearing), we can define the total distinguishability as

$$
S_{\sigma}=\sqrt{\sum_{i j} S_{i j}^{2}}=\sqrt{\sum_{i j} \frac{\left(N_{i j}^{\delta}-N_{i j}^{0}\right)^{2}}{\sigma_{i j}^{2}}},
$$

where the sum is over all the bins.

Although the correlated systematic errors, e.g., those of the overall flux normalization and the tilt of the spectrum, do not reproduce the pattern of the distribution for different values of $\delta$, still they can reduce significance substantially. Effects of the correlated errors will be considered in section 6.2 .

We will avoid precise statistical interpretation of distinguishability and just consider that it gives some idea about significance and sensitivity. Still, in various cases $|S|$ turns out to be close to the significance as follows from comparison of our previous estimations with results of complete MC simulations [24, 34]. Furthermore it reproduces rather precisely dependences of sensitivities on characteristics of detectors and neutrino parameters.

Apart from the total distinguishability, the sensitivity can be characterized also by maximal positive and negative $\mathrm{CP}$-differences in individual bins in a given range of energies and zenith angles.

Of course, the $\chi^{2}$ or maximal likelihood analyses would give higher quality, more reliable and precise estimation of the sensitivity. For this, however, one needs to perform Monte Carlo (MC) simulation of events at Super-PINGU. We are certainly not in position to make these simulations and this is beyond the scope of this paper.

\section{Distributions of the $\nu_{\mu}$ events}

\subsection{The density of $\nu_{\mu}$ events}

The $\nu_{\mu}$ (track) events produced mainly by the charged current $\nu_{\mu}$ interactions, $\nu_{\mu}+N \rightarrow$ $\mu+X, \bar{\nu}_{\mu}+N \rightarrow \mu^{+}+X$, are observed as muon tracks accompanied by hadronic cascades. For these events the energy of the muon $E_{\mu}$ and the direction of its trajectory characterized by the angles $\theta_{\mu}$ and $\phi_{\mu}$ as well as the total energy of the hadronic cascade (for deep-inelastic scattering) $E_{h}$ can be measured. Using this information one can reconstruct the neutrino 
energy as

$$
E_{\nu}^{r} \approx E_{\mu}+E_{h}-m_{N},
$$

where $m_{N}$ is the nucleon mass. Also the direction of cascade can be determined to some extent. So one can reconstruct the neutrino direction.

At low energies processes with one pion resonance production is important and below $1 \mathrm{GeV}$ the quasi-elastic scattering dominates. For these events procedure of reconstruction of the neutrino energy and direction becomes different. So, the detection of the low energy events should be considered separately, and such a study is beyond the scope of this paper. There are also some contributions from $\nu_{\tau}$ which produce $\tau$ leptons with subsequent decay into muons. In this initial study for estimations we extrapolate characteristics of reconstruction functions determined at high energies down to low energies.

The $\delta$-dependent part of the number density of the $\nu_{\mu}$ events in a single bin equals

$$
d_{\mu}^{\delta} \equiv \sigma^{\mathrm{CC}} \Phi_{\mu}^{0}\left[\left(P_{\mu \mu}^{\delta}+\frac{1}{r} P_{e \mu}^{\delta}\right)+\kappa_{\mu}\left(\bar{P}_{\mu \mu}^{\delta}+\frac{1}{\bar{r}} \bar{P}_{e \mu}^{\delta}\right)\right] .
$$

where

$$
\kappa_{\mu} \equiv \frac{\bar{\sigma}^{\mathrm{CC}} \bar{\Phi}_{\mu}^{0}}{\sigma^{\mathrm{CC}} \Phi_{\mu}^{0}}, \quad r \equiv \frac{\Phi_{\mu}^{0}}{\Phi_{e}^{0}}, \quad \bar{r} \equiv \frac{\bar{\Phi}_{\mu}^{0}}{\bar{\Phi}_{e}^{0}} .
$$

The ratios $r$ and $\bar{r}$ depend both on the neutrino energy and zenith angle, e.g., in the range $E_{\nu}=(2-25) \mathrm{GeV}$ and for $\cos \theta_{z}=-0.8$ the ratio can be roughly parameterized as $r=1.2 \cdot\left(E_{\nu} / 1 \mathrm{GeV}\right)^{0.65}$. Below $2 \mathrm{GeV}$ one has $r \approx 2$.

From eqs. (2.3) and (2.4) we find for neutrino contribution

$$
\begin{aligned}
d_{\mu}^{\delta}-d_{\mu}^{0}= & \sigma^{\mathrm{CC}} \frac{1}{r} \Phi_{\mu}^{0} \sin 2 \theta_{23}\left|A_{e \tilde{2}} A_{e \tilde{3}}\right|[(r-1) \cos \phi(1-\cos \delta)-\sin \phi \sin \delta] \\
& +\sigma^{\mathrm{CC}} \Phi_{\mu}^{0} D_{23}\left(1-\frac{1}{\cos \delta}\right) .
\end{aligned}
$$

This shows that in the case of $D_{23}=0$ the difference $d_{\mu}^{\delta}-d_{\mu}^{0}$ should vanish whenever $A_{e \tilde{2}}=0$ or $A_{e \tilde{3}}=0$, i.e. along the solar and atmospheric magic lines for probabilities considered above. The antineutrino contribution can be written similarly. It is suppressed in comparison to the neutrino contribution by factor $\sim 0.2$ due to smaller probabilities (factor of 2 at low energies, see figure 2) and smaller cross-section.

Notice that the main sensitivity to the mass hierarchy searches comes from $P_{e \tilde{3}}$ which is screened at low energies where $r=2$. In contrast, no screening of the CP-dependent terms occurs. The phase $\delta$ affects relative contribution of the $\nu_{e}-\nu_{\mu}$ and $\nu_{\mu}-\nu_{\mu}$ channels.

The fine-binned, $\Delta\left(\cos \theta_{z}\right)=0.025$ and $\Delta E_{\nu}=0.5 \mathrm{GeV}$, distribution of CP differences of $\nu_{\mu}$ events in Super-PINGU (3.8) for different values of $\delta$ are shown in figure 3. Here contributions from $\nu$ and $\bar{\nu}$ are summed up.

Let us consider dependence of the distributions of events on $\delta$ given in (4.2) which is explicit and exact. The first term in brackets of (4.2) as function of $\delta$ is symmetric with respect to $\delta=\pi$, whereas the second one is antisymmetric. The relative contributions of the two terms are determined by the phase $\phi$. As we will see, the first term dominates and the second one produces shift of maximum of $S_{\sigma}$ to $\delta>\pi$. 
The patterns of distributions of events are determined by the domain structure formed by the magic lines. Namely, the borders of domains are inscribed in the grid of magic lines with interconnections in the resonance regions [22]. Non-zero value of $D_{23}$ produces further shift of borders.

Let us consider the magic lines for the difference of densities of events for a given $\delta$ and $\delta=0$. Since $A_{e \tilde{3}}$ and $A_{e \tilde{2}}$ appear as common factors (in the approximation of $D_{23}=0$ ) the solar and atmospheric magic lines are the same as for the probabilities if neutrinos and antineutrinos are considered separately. The exact interference phase condition for number of events corresponds to zero value of the terms in the brackets of (4.2) which gives

$$
\tan \phi \approx-\tan \phi_{31}^{m} \approx \frac{(r-1)(1-\cos \delta)}{\sin \delta} .
$$

At high energies the $\nu_{\mu}$ flux dominates $(r \gg 1)$ and the pattern of the $d_{\mu}$ distribution follows dependence of the probability $P_{\mu \mu}$ on $E_{\nu}$ and $\cos \theta_{z}$, in particular the $P_{\mu \mu}$ domain structure. For $E_{\nu} \gtrsim 3 \mathrm{GeV}$ one can clearly see three solar (vertical) magic lines at $\cos \theta_{z}$ presented in (2.30). The interference phase condition is given in (2.33) with $\phi(\delta)$ obtained from (4.3). The oblique lines with different values of the slope parameter $A$ in figure 3 correspond to $\phi=\phi_{\min }, \phi=\phi_{\min }+\pi, \phi=\phi_{\min }+2 \pi$, etc. E.g., for $\delta=\pi$, we obtain from (4.3) $\phi_{\min }=\pi / 2$, and the lines correspond to $A=25,8.5$ and $5 \mathrm{GeV}$. The phase $\phi$ and consequently, the slopes change slightly with $\delta$. In the range below $6 \mathrm{GeV}$ the pattern of distribution is also determined by the atmospheric magic lines. The pattern is also affected by non-zero $D_{23}$ as well as by contribution from antineutrinos, which have shifted magic lines with respect to the neutrino lines.

With increase of $\delta$ the domain structure does not change qualitatively although the domains of the negative CP-difference (blue) expand, especially the one which is aligned to the magic line (2.33) with $A=25 \mathrm{GeV}$. The values of CP-differences increase and asymmetry between negative and positive $\mathrm{CP}$ differences increases with $\delta$, as one can read from numbers at the explanatory bars. Maximal CP phase effect is in the lowest energy bins.

At low energies $r \approx 2$, and the difference of the densities equals

$$
d_{\mu}^{\delta}-d_{\mu}^{0}=\frac{1}{2} \sigma^{\mathrm{CC}} \Phi_{\mu}^{0} \sin 2 \theta_{23}\left|A_{e \tilde{2}} A_{e \tilde{3}}\right|[\cos \phi(1-\cos \delta)-\sin \phi \sin \delta] .
$$

Effect of averaging over $E_{\nu}$ and $\theta_{z}$ can be explored using the constant density approximation. From (2.20) we obtain for the neutrino part (the first term in (4.1))

$$
\left\langle d_{\mu}^{\delta}\right\rangle-\left\langle d_{\mu}^{0}\right\rangle=-\sigma^{\mathrm{CC}} \Phi_{\mu}^{0} \frac{J_{\theta}}{2}\left[\left(1-\frac{1}{r}\right)(1-\cos \delta) \sin ^{2} \phi_{21}^{m}-\frac{1}{2 r} \sin \delta \sin 2 \phi_{21}^{m}\right] .
$$

At low energies, when $r \approx 2$, this expression reduces to

$$
\left\langle d_{\mu}^{\delta}\right\rangle-\left\langle d_{\mu}^{0}\right\rangle=-\sigma^{\mathrm{CC}} \Phi_{\mu}^{0} \frac{J_{\theta}}{4}\left[(1-\cos \delta) \sin ^{2} \phi_{21}^{m}-\frac{1}{2} \sin \delta \sin 2 \phi_{21}^{m}\right],
$$

where the additional factor $1 / 2$ is due to the flavor suppression. Comparing (4.6) and (4.4) we find that averaging is reduced to substitution in the CP-factor $\cos \phi \rightarrow \sin ^{2} \phi_{21}^{m}$ and 

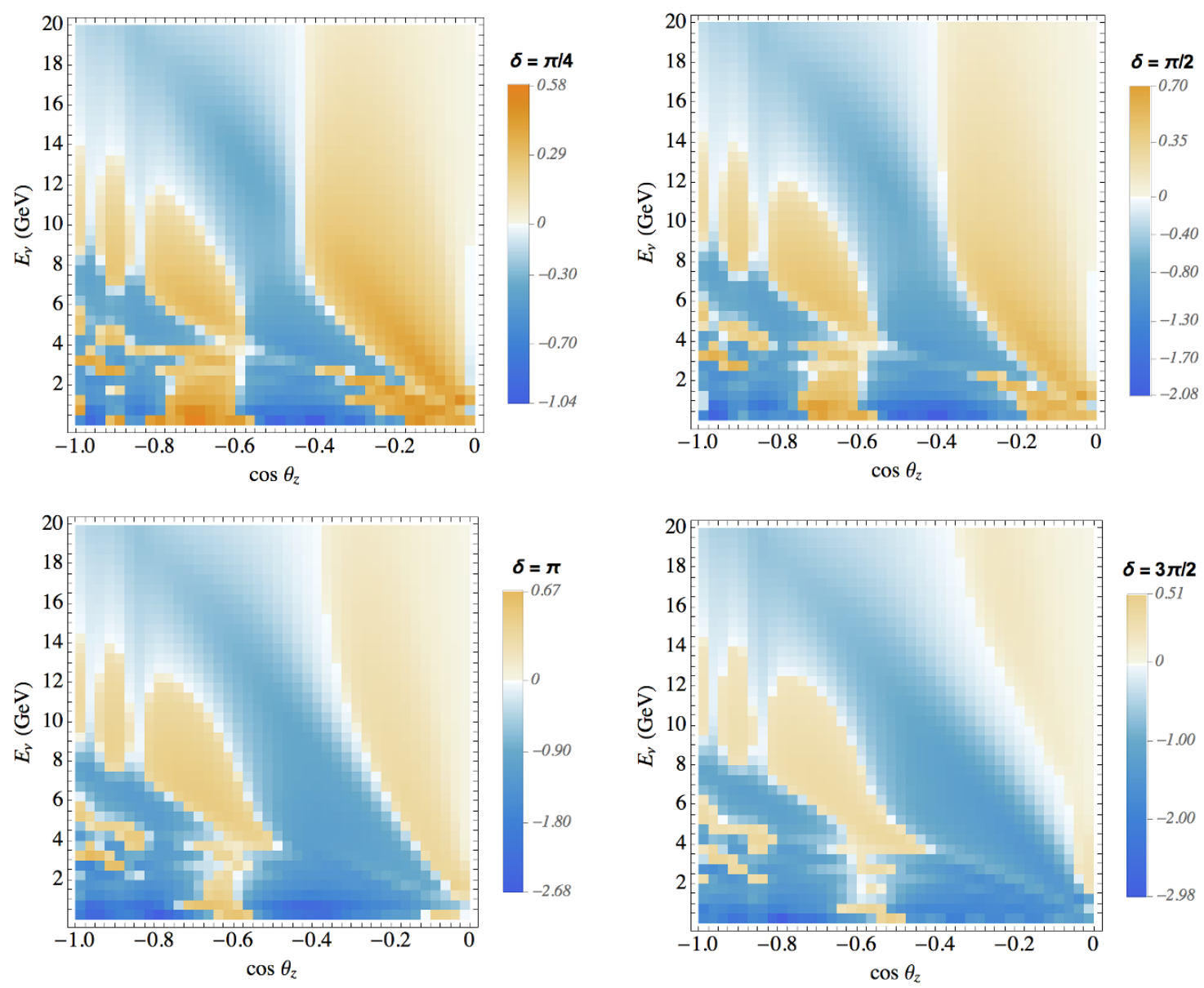

Figure 3. Distributions of the relative CP differences, $S_{i j}(f=0) \equiv\left(N_{i j}^{\delta}-N_{i j}^{0}\right) / \sqrt{N_{i j}(\delta=0)}$, for the $\nu_{\mu}+\bar{\nu}_{\mu}$ events in the $E_{\nu}-\cos \theta_{z}$ plane. 1-year of exposure of Super-PINGU has been used. The CP difference is given between $\delta=0$ and $\delta=\pi / 4$ (top left panel), $\delta=\pi / 2$ (top right panel), $\delta=\pi$ (bottom left panel) and $\delta=3 \pi / 2$ (bottom right panel). Note that the lowest energy bin is $(0.2-0.5) \mathrm{GeV}$. Normal neutrino mass hierarchy is assumed.

$\sin \phi \rightarrow 0.5 \sin 2 \phi_{21}^{m}$. Notice that dependence of the differences on $E_{\nu}$ and $\theta_{z}$ is in $\phi_{21}^{m}, \Phi_{\mu}^{0}$ and $r$. Expression in (4.6) is a combination of two functions: $\sin ^{2} \phi_{21}^{m}$ and $\sin 2 \phi_{21}^{m}$ with weights determined by the phase $\delta$. The first function is even and the second is odd in $\delta$ and both functions vanish along the magic lines. For several specific values of $\delta$ we obtain (in units $\frac{1}{4} \sigma^{\mathrm{CC}} \Phi_{\mu}^{0} J_{\theta}$ )

$$
\begin{aligned}
\left\langle d_{\mu}^{\pi / 4}\right\rangle-\left\langle d_{\mu}^{0}\right\rangle & \propto-\left(1-\frac{1}{\sqrt{2}}\right) \sin ^{2} \phi_{21}^{m}+\frac{1}{2 \sqrt{2}} \sin 2 \phi_{21}^{m} \\
\left\langle d_{\mu}^{\pi / 2}\right\rangle-\left\langle d_{\mu}^{0}\right\rangle & \propto-\sin ^{2} \phi_{21}^{m}+\frac{1}{2} \sin 2 \phi_{21}^{m} \\
\left\langle d_{\mu}^{\pi}\right\rangle-\left\langle d_{\mu}^{0}\right\rangle & \propto-2 \sin ^{2} \phi_{21}^{m} \\
\left\langle d_{\mu}^{3 \pi / 2}\right\rangle-\left\langle d_{\mu}^{0}\right\rangle & \propto-\sin ^{2} \phi_{21}^{m} \frac{1}{2} \sin 2 \phi_{21}^{m}
\end{aligned}
$$


Similarly according to (2.27) and (2.28) the difference for antineutrinos equals

$$
\left\langle\bar{d}_{\mu}^{\delta}\right\rangle-\left\langle\bar{d}_{\mu}^{0}\right\rangle=\sigma^{\mathrm{CC}} \bar{\Phi}_{\mu}^{0} \frac{\bar{J}_{\theta}}{2}\left[(1-\cos \delta) \sin ^{2} \bar{\phi}_{21}^{m}\left(1-\frac{1}{\bar{r}}\right)-\frac{1}{2 \bar{r}} \sin \delta \sin 2 \bar{\phi}_{21}^{m}\right] .
$$

If mixings and phases in neutrino and antineutrino channels are approximately equal, the effect of inclusion of antineutrinos could be accounted for by the overall suppression factor $1-\langle\bar{P}\rangle /\langle P\rangle \kappa_{\mu} \approx 0.8$ to that of neutrino only without change of the shape of the distribution. Differences of the phases and mixing angles in the neutrino and antineutrino channels lead to distortion of the neutrino distribution mainly in the regions around the magic lines.

The $\nu_{\tau}$ - flux appears at the detector due to the $\nu_{\mu}-\nu_{\tau}$ oscillations. In turn, the $\nu_{\tau}$ interactions $\nu_{\tau}+N \rightarrow \tau+h \rightarrow \mu+\nu_{\tau}+\nu_{\mu}+h$ will contribute to the sample of $\nu_{\mu}$-events with a muon and a hadron cascade in the final state. However, the number of these events is relatively small due to its small branching ratio and small cross-section of the $\tau$ production near the energy threshold. Also these events have certain features which can be used to discriminate them from the true $\nu_{\mu}$-events [24]. As we will see, the highest sensitivity to $\delta$ is in the sub-GeV region where $\tau$ leptons are not produced. In our simplified study the effect of the $\tau$ decays can be accounted by adding a systematic error.

\subsection{Smearing}

In figure 4 we show the relative CP-difference distributions of the $\nu_{\mu}$ events, $S_{i j}(f=0)$ in (3.12), smeared with the Super-PINGU reconstruction functions as defined in section III B. Smearing leads to disappearance of fine structures and to merging of regions of the same sign CP-differences. There is dominant region of the negative $\mathrm{CP}$-differences $S_{i j}<0$ and two separate regions with $S_{i j}>0$. The large one is at $\left|\cos \theta_{z}\right|<0.4$ and at high energies restricted from below by the first interference phase line. The smaller region is at $\cos \theta_{z}<-0.6$ and energies $E=(6-14) \mathrm{GeV}$. Actually, it consists of three regions situated between the solar (vertical) magic lines and between two lines (second and third) determined by the interference phase conditions.

With increase of $\delta$ the region with $S_{i j}<0$ expands. Correspondingly, the region of positive $S$ at $\left|\cos \theta_{z}\right|<0.4$ shifts to higher energies, whereas the smaller region slightly shrinks. Asymmetry between maximal positive and maximal negative CP-differences increases. Below $5 \mathrm{GeV}$ the region of negative $S$ expands to horizontal directions (larger $\cos \theta_{z}$ ) and for $\delta \sim(1-1.5) \pi$ we find $S_{i j}<0$ for all $\cos \theta_{z}$. At low energies and the bins with the highest CP-difference are in the zenith angle intervals $\left|\cos \theta_{z}\right|=0.9-1.0$ and $\left|\cos \theta_{z}\right|<0.3-0.5$. For $\delta>\pi / 2$ the dip near vertical directions is larger than in outer regions. With increase of $\delta$ the latter shifts from $\cos \theta_{z}=-0.43$ to -0.3 . It is these features that should be used to measure $\delta$.

Smearing leads to a substantial decrease of the sensitivity to $\delta$. This reduction is a consequence of the integration over regions with different values and signs of $S_{i j}$. The decrease of sensitivity is characterized by factors $(2.3-1.7)$ for small $\delta \sim(0.25-0.5) \pi$ and by factor 1.3 in the range $\delta \sim(1-1.5) \pi / 2$, where the CP phase effect is large (see also section 6 and figure 9$)$. The reason is that for small $\delta$ at low energies the regions with $S_{i j}>0$ and $S_{i j}<0$ are comparable in size and in absolute values of $S_{i j}$ (see figures 3 

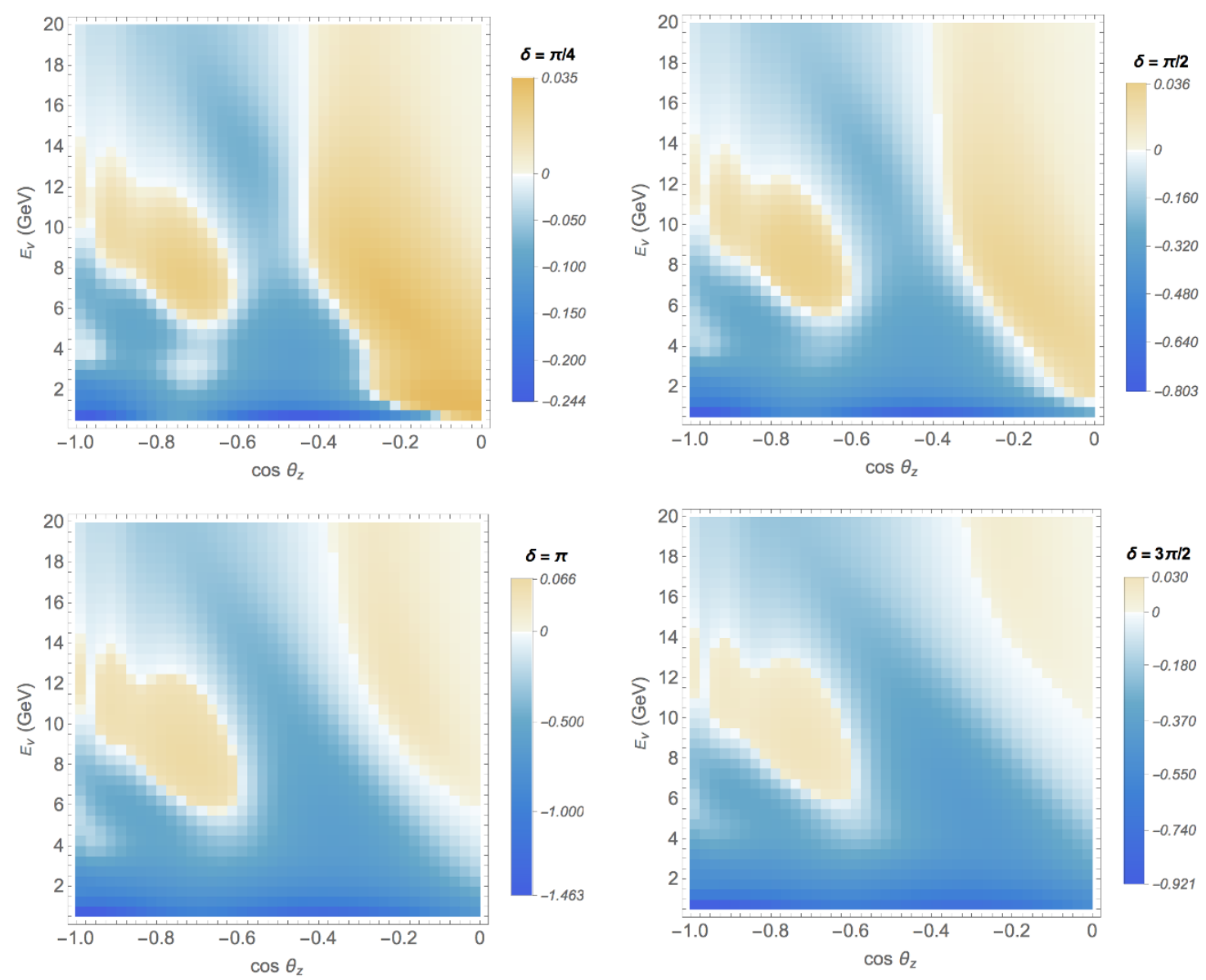

Figure 4. The same as in figure 3, but after smearing of the distributions over the energy and zenith angle of neutrinos. The smearing functions have been taken in the form of the PINGU reconstruction functions with widths reduced by factor $1 / \sqrt{3}$.

and 4). So that smearing (integration over the energy and zenith angle) leads to partial cancellation. With increase of $\delta$ the asymmetry between the regions with positive and negative $S_{i j}$ increases, thus reducing the cancellation.

Smearing with PINGU reconstruction functions leads to stronger decrease of sensitivity, mostly in the $\delta<\pi$ region (see section 6 ).

To evaluate contributions to the total distinguishability $S_{\sigma}$ from different energy regions we have computed $S_{\sigma}\left(E_{\mathrm{th}}\right)$ for 1 year of exposure and fixed $V_{\text {eff }}\left(E_{\nu}\right)$ using different minimal energies of integration, $E_{\text {th }}$. Here $f=0$, i.e., the systematic errors have not been included.

We find that with decrease of $E_{\text {th }}$ from $1 \mathrm{GeV}$ down to $0.5 \mathrm{GeV} S_{\sigma}$ increases by a factor (1.5-1.7) depending on the value of $\delta$. Decrease of $E_{\text {th }}$ from $0.5 \mathrm{GeV}$ down to $0.2 \mathrm{GeV}$ leads to increase of $S_{\sigma}$ by another factor (1.3-1.9), with strongest increase at small values of $\delta$. It should be noticed that extrapolation of the results below $0.5 \mathrm{GeV}$ becomes unreliable. 

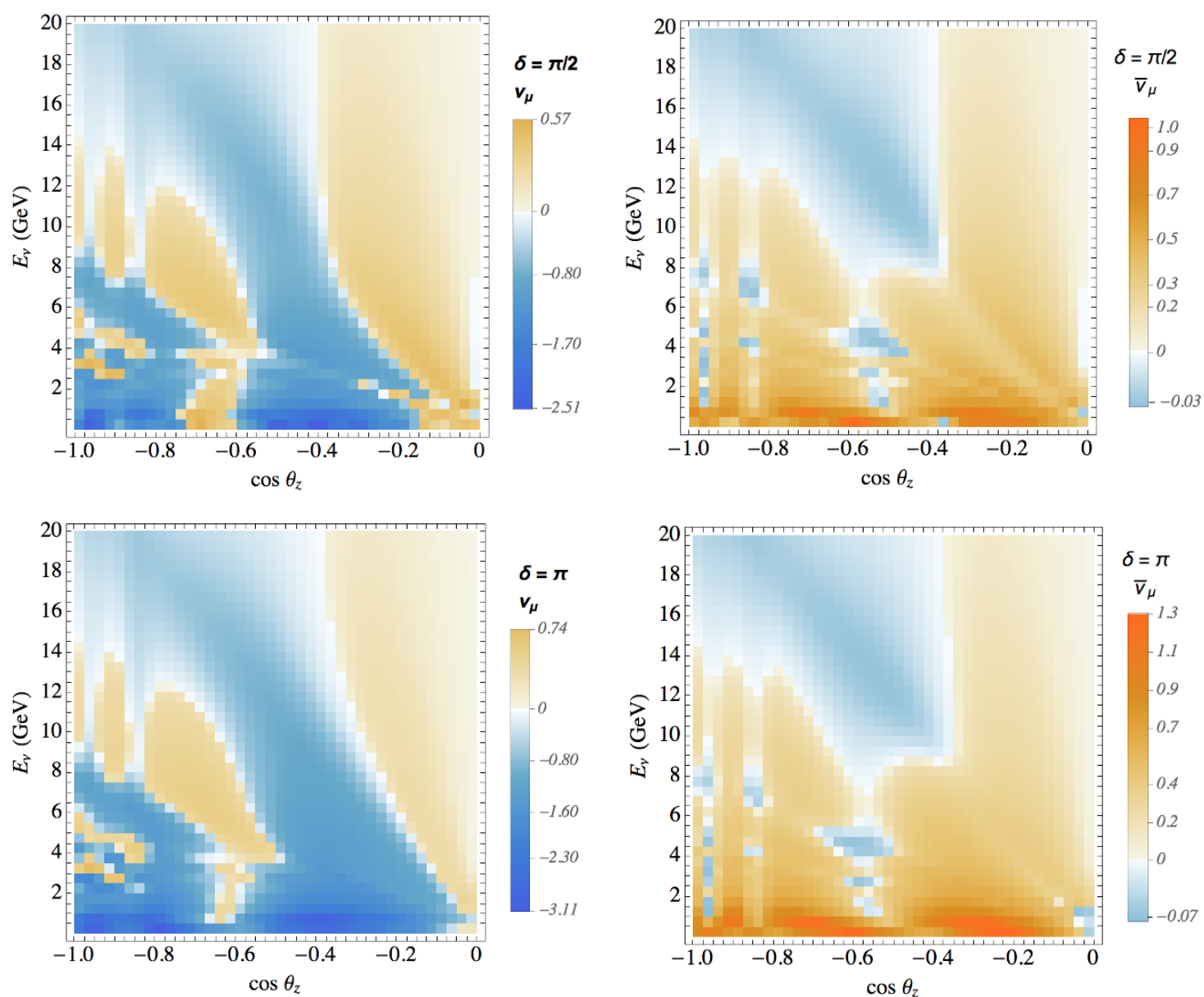

Figure 5. The same as in figure 3, but separately for $\nu_{\mu}$ and $\bar{\nu}_{\mu}$ events and for values of the CP phase $\delta=\pi / 2$ (upper panels) and $\delta=\pi$ (lower panels).

\subsection{Neutrinos and antineutrinos}

Measurements of the inelasticity ( $y$-distribution) allow to make partial separation of the neutrino and antineutrino signals on statistical ground [48]. Since the CP-differences have opposite signs for neutrinos and antineutrinos, at least at low energies, one expects improvement of sensitivity to the CP-phase if the $\nu$ and $\bar{\nu}$ signals are separated. To assess the possible improvement we consider first the ideal situation of complete separation. In figure 5 we show the unsmeared CP-difference plots for neutrinos and antineutrinos separately. We take $\delta=\pi / 2$ and $\delta=\pi$.

For neutrinos (resonance channel) the distribution is rather similar to that for the sum of $\nu$ and $\bar{\nu}$ signals. Asymmetry between positive and negative contributions increases with exclusion of $\bar{\nu}$. As a result, the significance for neutrinos alone increases by a factor (1.3-1.4) in comparison with significance for the sum of the signals.

In contrast, the distribution for antineutrinos shows different pattern: it has the same sign of the CP difference, $S_{i j}$, (positive or negative in the same regions) as for neutrinos 
in high energy region, $E>8 \mathrm{GeV} .^{2}$ Whereas at low energies the antineutrino and neutrino distributions have opposite signs, and so cancellation is strong in the absence of separation. For antineutrinos, especially at low energies, the positive asymmetry dominates. This can be immediately understood on the basis of our analytic consideration in section 2.3.

The distinguishability without separation is larger than the difference of $\nu$ and $\bar{\nu}$ distinguishabilities. For $E_{\text {th }}=0.5 \mathrm{GeV}$ and $\delta=\pi$ we have $S_{\sigma, \nu}=13.5, S_{\sigma, \bar{\nu}}=5.8$ and $S_{\sigma, \nu}-S_{\sigma, \bar{\nu}}=7.7$, which is smaller than total $S_{\sigma}=9.9$. This means that cancellation is not complete and reflects the fact that in the high energy regions the sign of CP-difference is the same for $\nu$ and $\bar{\nu}$. There is an asymmetry between the neutrino and antineutrino contributions related to difference of the cross-sections and fluxes. In the case of ideal separation we would have $S_{\sigma}=14.7$, instead of 9.9, i.e. almost 1.5 times larger than without separation. Smearing and partial separation will reduce this enhancement factor substantially. Separation of neutrinos and antineutrinos, i.e. reconstruction of $y$-distributions, is possible at high energies $E>3 \mathrm{GeV}$. At low energies that becomes problematic.

\subsection{Inverted mass hierarchy}

For the inverted neutrino mass hierarchy (IH) the pattern of distributions is inverted with respect to that for the normal mass hierarchy at high energies and it is the same for low energies, see figure 6 . The difference is related to the 1-3 resonance whose effect is different for normal and inverted hierarchies. At low energies sensitivity to the mass hierarchy disappears.

Formally all the expressions for probabilities and amplitudes in terms of mixing angles and phases (eigenvalues) in matter are the same as in the case of $\mathrm{NH}$ but values of the angles and phases change. Also the signs of the phases $\phi_{31}^{m}$ and $\phi_{32}^{m}$ change. Since the 1-2 mass ordering does not change, averaging over $\phi_{32}^{m}$ and $\phi_{31}^{m}$ will give at low energies the same expression for the probabilities with the only change $J_{\theta} \rightarrow J_{\theta}^{\mathrm{IH}}$, and in $J_{\theta}^{\mathrm{IH}}$ only $\theta_{13}^{m}$ changes. Thus, for densities of events we obtain

$$
\frac{d^{\mathrm{IH}}}{d^{\mathrm{NH}}} \approx \frac{J_{\theta}^{\mathrm{IH}}}{J_{\theta}^{\mathrm{NH}}} \approx \frac{\sin 2 \theta_{13}^{m \mathrm{IH}} \cos \theta_{13}^{m I H}}{\sin 2 \theta_{13}^{m \mathrm{NH}} \cos \theta_{13}^{m \mathrm{NH}}} .
$$

Furthermore, for energies much below the 1-3 resonance energy, $\theta_{13}^{m \mathrm{IH}} \approx \theta_{13}^{m \mathrm{NH}} \approx \theta_{13}$. So that the densities of events in both cases are expected to be approximately equal.

We find that the integral distinguishabilities of the same phases for the inverted hierarchy is about (25-30)\% lower. Also the $\nu-\bar{\nu}$ separation is more important for inverted mass hierarchy since in this case the difference of signals from neutrinos and antineutrinos is smaller.

\section{Cascade events}

\subsection{Density of events}

The cascade events are produced by the CC $\nu_{e}$ interactions $\nu_{e}+N \rightarrow e+X, \bar{\nu}_{e}+N \rightarrow e^{+}+X$ and several other processes (see discussion below). The density of the CC $\nu_{e}$ events is given

\footnotetext{
${ }^{2}$ This is consequence of the level crossing in the neutrino channel.
} 

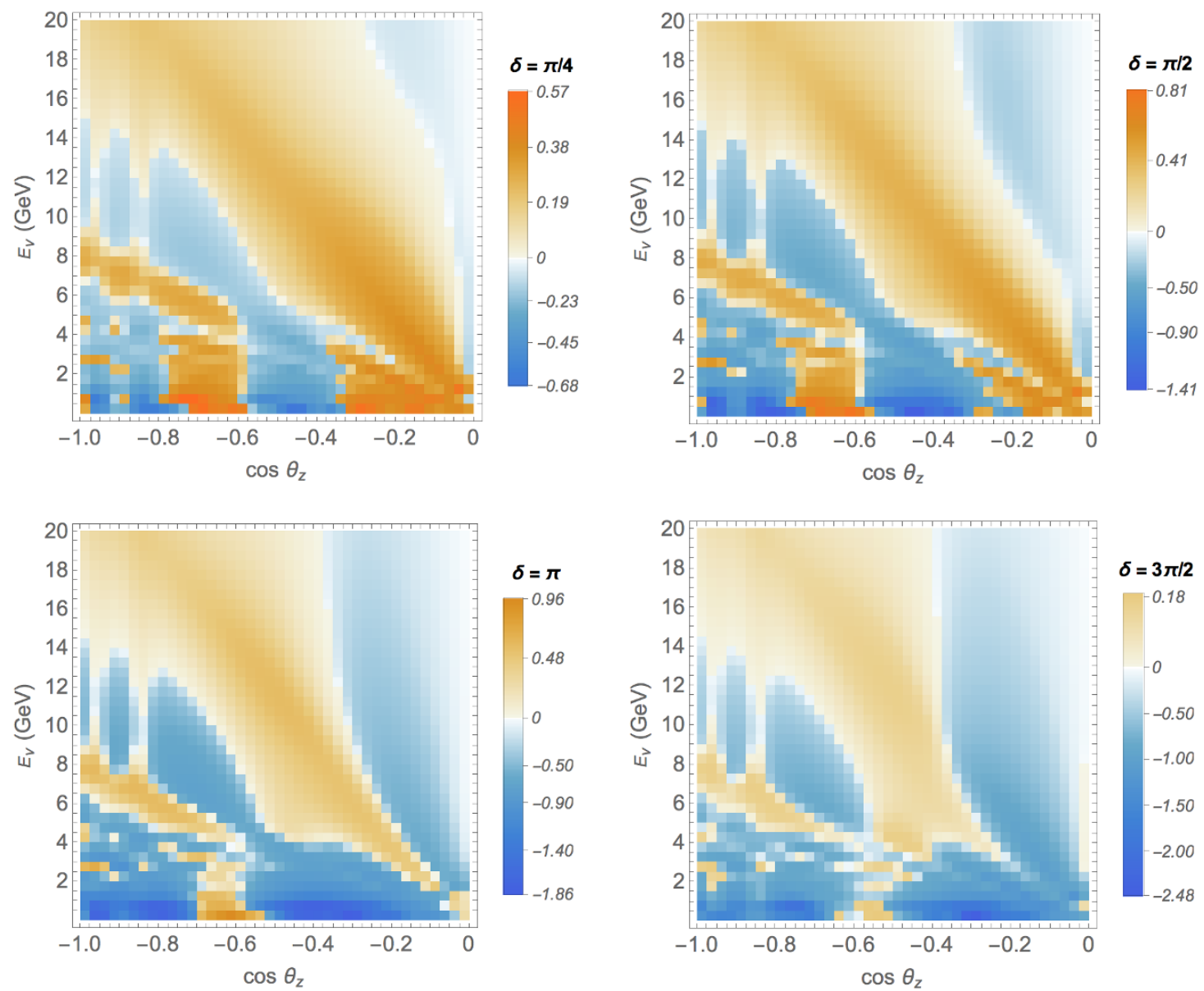

Figure 6. The same as in figure 3, but for the inverted neutrino mass hierarchy.

by

$$
d_{e}\left(E, \cos \theta_{z}\right)=\sigma^{\mathrm{CC}} \Phi_{e}^{0}\left[\left(P_{e e}+r P_{\mu e}\right)+\kappa_{e}\left(\bar{P}_{e e}+\bar{r} \bar{P}_{\mu e}\right)\right]
$$

where $\kappa_{e} \equiv\left(\bar{\sigma}_{e} / \sigma_{e}\right)\left(\bar{\Phi}_{\mu}^{0} / \Phi_{\mu}^{0}\right)$. Its $\delta$-dependent part,

$$
d_{e}^{\delta} \equiv \sigma^{\mathrm{CC}} \Phi_{\mu}^{0}\left[P_{\mu e}^{\delta}+\kappa_{e} \bar{P}_{\mu e}^{\delta}\right] \approx \sigma^{\mathrm{CC}} \Phi_{\mu}^{0}\left[\left|A_{e \tilde{3}} A_{e \tilde{2}}\right| \cos (\phi-\delta)+\kappa\left|\bar{A}_{e \tilde{3}} \bar{A}_{e \tilde{2}}\right| \cos (\bar{\phi}+\delta)\right]
$$

is determined by the $\nu_{\mu} \rightarrow \nu_{e}$ oscillation probability only, since $P_{e e}$ and $\bar{P}_{e e}$ are $\delta$ independent. Consequently, there is no flavor suppression of the CP-violation effects even for low energies.

The difference of densities of the $\nu_{e}$-events equals

$$
d_{e}^{\delta}-d_{e}^{0} \approx-\sigma^{\mathrm{CC}} \Phi_{\mu}^{0} \sin 2 \theta_{23}\left|A_{e \tilde{3}} A_{e \tilde{2}}\right|[\cos \phi(1-\cos \delta)-\sin \phi \sin \delta]
$$

As we discussed before, the antineutrino contribution is suppressed by a factor $1 / 4$. Comparing expression (5.2) with (4.2) and (4.4) we find that for $r \approx 2$ and $D_{23} \approx 0$,

$$
d_{e}^{\delta}-d_{e}^{0}=-2\left(d_{\mu}^{\delta}-d_{\mu}^{0}\right) .
$$


So, the $\mathrm{CP}$ difference of the $\nu_{e}$ events has an opposite sign with respect to the $\mathrm{CP}$ difference of the $\nu_{\mu}$ events and its size is two times larger. As a result, the cascade events can give even bigger contribution to distinguishability of different values of $\delta$ than the $\nu_{\mu}$ events. The reason is the flavor suppression of CP-differences for the $\nu_{\mu}$ events, which is absent for the $\nu_{e}$ events. With increase of energy the ratio $r$ increases, the flavor suppression becomes weaker. Consequently, $\left(d_{\mu}^{\delta}-d_{\mu}^{0}\right)$ increases and the numerical factor in equation (5.3) becomes smaller, approaching 1. This increase depends on the value of $\delta$.

Explicit expression for the density of events averaged over $\phi_{32}^{m}$ (which is valid at low energies) can be obtained using the constant density approximation. Since $P_{\mu e}=P_{e \mu}(\delta \rightarrow$ $-\delta$ ) we have from $(2.18)$

$$
\left\langle P_{\mu e}^{\delta}\right\rangle=\frac{J_{\theta}}{2}\left[\cos \delta \cos 2 \theta_{12}^{m} \sin ^{2} \phi_{21}^{m}-\frac{1}{2} \sin \delta \sin 2 \phi_{21}^{m}\right] .
$$

Correspondingly, when $\cos 2 \theta_{12}^{m} \approx-1$, the difference of probabilities for a given $\delta$ and $\delta=0$ equals

$$
\left\langle P_{\mu e}^{\delta}\right\rangle-\left\langle P_{\mu e}^{0}\right\rangle=\frac{J_{\theta}}{2}\left[(1-\cos \delta) \sin ^{2} \phi_{21}^{m}-\frac{1}{2} \sin \delta \sin 2 \phi_{21}^{m}\right] .
$$

For antineutrinos such a difference has similar expression but with overall minus sign and mixing angles and phases in matter taken for antineutrinos.

For difference of the densities of events we obtain

$$
\begin{aligned}
\left\langle d_{e}^{\delta}\right\rangle-\left\langle d_{e}^{0}\right\rangle= & \sigma^{\mathrm{CC}} \Phi_{\mu}^{0} \frac{J_{\theta}}{2}\left[(1-\cos \delta) \sin ^{2} \phi_{21}^{m}-\frac{1}{2} \sin \delta \sin 2 \phi_{21}^{m}\right] \\
& -\bar{\sigma}^{\mathrm{CC}} \bar{\Phi}_{\mu}^{0} \frac{\bar{J}_{\theta}}{2}\left[(1-\cos \delta) \sin ^{2} \bar{\phi}_{21}^{m}-\frac{1}{2} \sin \delta \sin 2 \bar{\phi}_{21}^{m}\right] .
\end{aligned}
$$

In the range where the phases of neutrinos and antineutrinos are approximately equal we have

$$
\left\langle d_{e}^{\delta}\right\rangle-\left\langle d_{e}^{0}\right\rangle \approx \sigma^{\mathrm{CC}} \Phi_{\mu}^{0} \frac{1}{2} J_{\theta}\left(1-\kappa \frac{\bar{J}_{\theta}}{J_{\theta}}\right)\left[(1-\cos \delta) \sin ^{2} \phi_{21}^{m}-\frac{1}{2} \sin \delta \sin 2 \phi_{21}^{m}\right],
$$

with the factor in the first brackets describing the C-suppression.

In figure 7 we show the unsmeared distribution of the CP differences of $\nu_{e}$ events $S_{i j}(f=0)$ for different values of $\delta$. As we marked, the transition probability $\nu_{\mu} \rightarrow \nu_{e}$ gives unique contribution to the $\mathrm{CP}$ difference, the $\nu_{e} \rightarrow \nu_{e}$ contribution and $D_{23}$ are absent. As a result, the distributions follow closely the domain structure of $\nu_{\mu} \rightarrow \nu_{e}$ probability determined by the magic lines. Now the interference phase lines depend on $\delta$. From (5.2) we obtain the condition for the phase, i.e., zero value of the expression in the brackets of eq. (5.2), as:

$$
\tan \phi \approx-\tan \phi_{31} \approx \frac{1-\cos \delta}{\sin \delta} .
$$

This condition does not depend on $r$ in contrast to (4.3), since only one transition probability enters. For $\delta=\pi / 2$ we obtain $\tan \phi=1$ or $\phi=\pi / 4+n \pi$. Now the pattern of distributions changes with $\delta$, since the interference phase and CP phase dependencies do not factor out. 

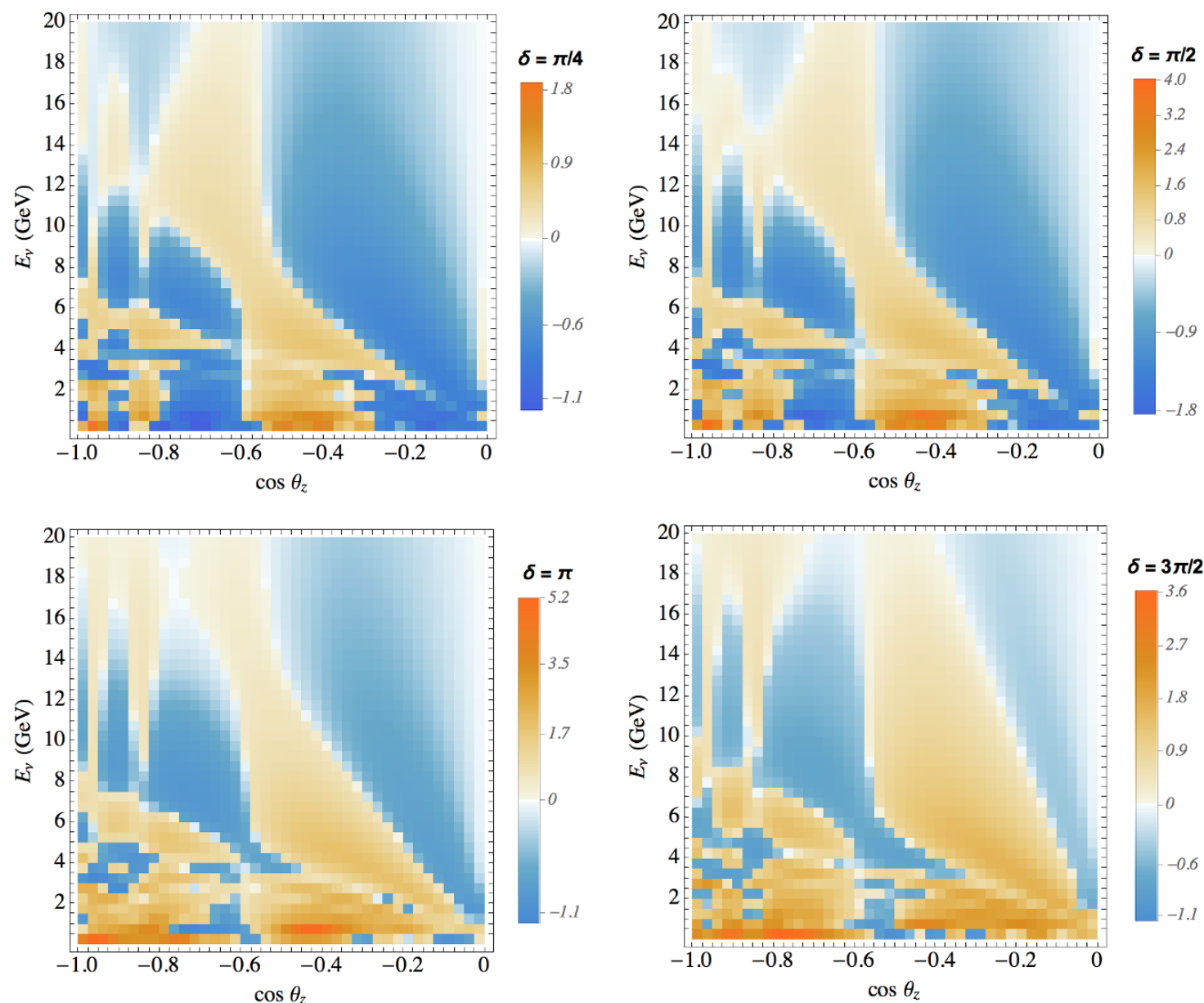

Figure 7. The same as in figure 3, but for the $\nu_{e}+\bar{\nu}_{e}$ events.

One can see in figure 7 three solar magic lines (2.30). The oblique structures are determined at high energies by the interference phase condition (2.33) with $\phi$ determined in (5.8). Pattern of the distribution is especially simple for $\delta=\pi$ when the equation (5.8) gives $\phi_{31}=\pi / 2+\pi n$ which coincides with the phase condition for probability. The oblique lines with the slope factor $A=25,8.2$ and $5 \mathrm{GeV}$ correspond to $\phi_{31}=\pi / 2,3 \pi / 2,5 \pi / 2$. With increase of $\delta$ the slopes of interference phase lines given by $A$ increase and so domains shift to higher energies. The upper right domain (high energies and small $\left|\cos \theta_{z}\right|$ ) is determined by the solar magic line and the first interference phase line, etc. At energies below the 1-3 resonance also the atmospheric magic lines determine the structure.

For the $\nu_{e}$ events, the pattern (regions of positive and negative CP-difference) is inverted in comparison to the pattern for the $\nu_{\mu}$ events in the whole energy range. So, good separation of the $\nu_{e}$ and $\nu_{\mu}$ events, i.e. flavor identification, is crucial (see section 6.3).

For the $\nu_{e}-$ events the positive CP-difference dominates. The total CPdistinguishability from $\nu_{e}$ - events is higher than from $\nu_{\mu}$, e.g., $S_{\sigma}\left(\nu_{e}\right)$ is a factor of $(1.5-1.8)$ bigger than $S_{\sigma}\left(\nu_{\mu}\right)$ for $E_{\text {th }}=0.5 \mathrm{GeV}$, with the biggest difference at small values of $\delta$. Decrease of the threshold from $1 \mathrm{GeV}$ down to 0.2 enhances distinguishability by a factor of $(1.3-1.5)$. 
The problem here is that the cascade events are not only due to $\nu_{e}$ interactions but also due to other processes which should be taken into account:

(i) Neutral current (NC) interactions of all types of neutrinos. These events are not affected by oscillations, and so do not contribute to the CP difference of the events. Still they increase the total number of events and therefore the statistical error in the denominator of $S_{\sigma}$ thus, diluting the significance. The NC contribution could be disentangled, if the hadron cascades are distinguished from the EM cascades induced by electrons. Notice that at low energies we deal with just few (1-2) individual pions and they can be distinguished from electrons.

(ii) The $\nu_{\tau}$ CC interactions which produce $\tau$ leptons. The latter generate cascades in all the decays of $\tau$ leptons but $\mu$. At low energies contribution of these events is suppressed due to high threshold of the $\tau$ lepton production.

(iii) Contribution of the CC $\nu_{\mu}$ events with faint muons (close to threshold of Cherenkov radiation). Fraction of these events is higher at low energies. A part of the CC $\nu_{e}$ events can be confused with the $\nu_{\mu}$ events when one of the pions will be misidentified with muon. This problem may be cured at least partly by introduction of additional kinematical cuts.

\subsection{Smearing of the cascade events}

In figure 8 we show results of smearing of cascades with the energy and angle reconstruction functions. We used the Super-PINGU reconstruction functions defined in section 3.2. Even after smearing one can see the CP-domain structure determined by the magic lines.

According to figure 8 the effect of smearing on distinguishability of the $\nu_{e}$ events is stronger than that of the $\nu_{\mu}$ events. The suppression is a factor (2.4-3.5) times in the interval from $\delta=(0.5-0.25) \pi$ and it is weaker for large phases: a factor $(1.4-1.5)$ in the interval $\delta=(1.5-1) \pi$. The pattern of the $S_{i j}$ distribution is inverse to that for $\nu_{\mu}$ events in a sense that $\left(E_{\nu}-\theta_{z}\right)$ regions with $S_{i j}<0$ for $\nu_{\mu}$ become regions with $S_{i j}>0$ for $\nu_{e}$. Below $5 \mathrm{GeV}$ with increase of $\delta$ the region with $S_{i j}>0$ expands towards the horizontal direction and for $\delta>\pi$ we find that $S_{i j}>0$ for all values of zenith angle. For fixed energy, two peak in the dependence of $S_{i j}$ on $\cos \theta_{z}$ are at $\cos \theta_{z} \sim-0.9$ and at $\left|\cos \theta_{z}\right| \sim(0.3-0.45)$.

At low energies, due to loss of angular resolution structures become essentially horizontal. Asymmetry between the positive and negative CP-differences increases with $\delta$ and $S_{i j}>0$ becomes dominant for $\delta \gtrsim \pi$. In spite of stronger smearing, the distinguishability of $\nu_{e}$ events is a factor (1.3-1.7) times larger (depending on value of $\delta$ and $E_{\text {th }}$ ) than the distinguishability of $\nu_{\mu}$ events.

\section{Sensitivity to the CP-phase}

In this section we will present the total distinguishability of different values of the CPphase and discuss how it can be affected by various systematic errors as well as flavor misidentification of events. This allows us to identify the main challenges of determination 

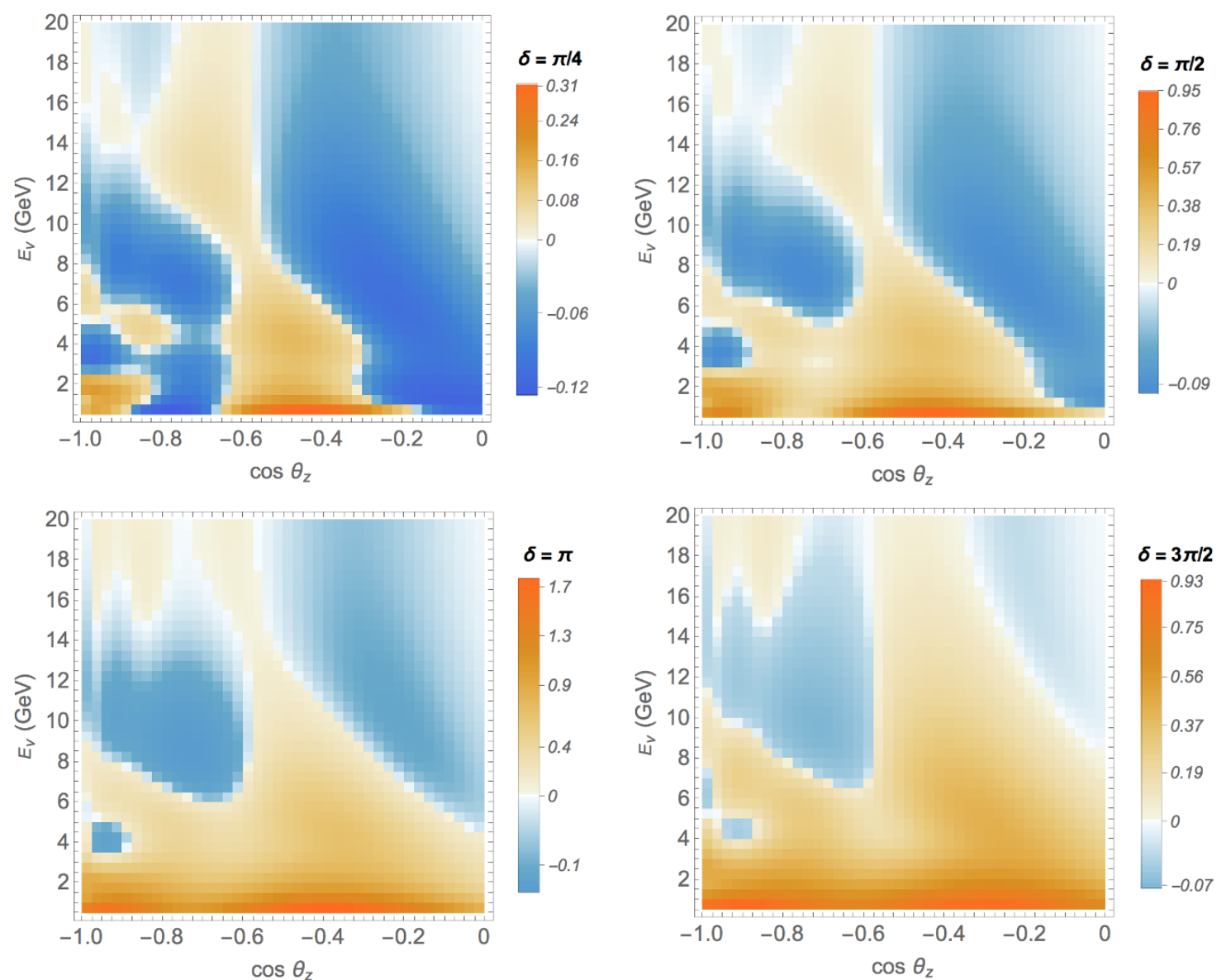

Figure 8. Same as in figure 4, but for the $\nu_{e}+\bar{\nu}_{e}$ events.

of $\delta$ and evaluate the level of admissible errors. We will comment on possible ways to reduce the errors and improve sensitivity to the $\mathrm{CP}$-phase.

\subsection{Total distinguishability and the energy threshold}

Figures 9 and 10 show the total distinguishabilities of a given value of the phase $\delta$ from $\delta=0$ for the $\nu_{\mu}$ and $\nu_{e}$ events, $S_{\sigma}\left(\nu_{\mu}\right)$ and $S_{\sigma}\left(\nu_{e}\right)$, after 1 year of exposure. We use thresholds $E_{\text {th }}=0.5 \mathrm{GeV}$ and $1.5 \mathrm{GeV}$ for Super-PINGU and $E_{\text {th }}=1.5 \mathrm{GeV}$ and $3 \mathrm{GeV}$ for PINGU. Shown are the dependence before smearing and after smearing over $E_{\nu}$ and $\theta_{z}$, with $f=0,2.5 \%$, and $5 \%$ uncorrelated systematic error. Let us comment on various features of the obtained distributions.

(i) Dependence of the distinguishabilities on $\delta$ before smearing is nearly symmetric with respect to $\pi$. Maximum is slightly shifted to $\delta<\pi$ for the $\nu_{e}$ events. Smearing diminishes $S_{\sigma}$, the suppression factor depends on $\delta$ and is different for the $\nu_{\mu}$ and $\nu_{e}$ events. As we discussed in section $4.2, S_{\sigma}$ is suppressed by smearing stronger for $\delta<\pi$. As a result, smearing shifts maximal sensitivity toward $\delta \approx 1.1 \pi$. 

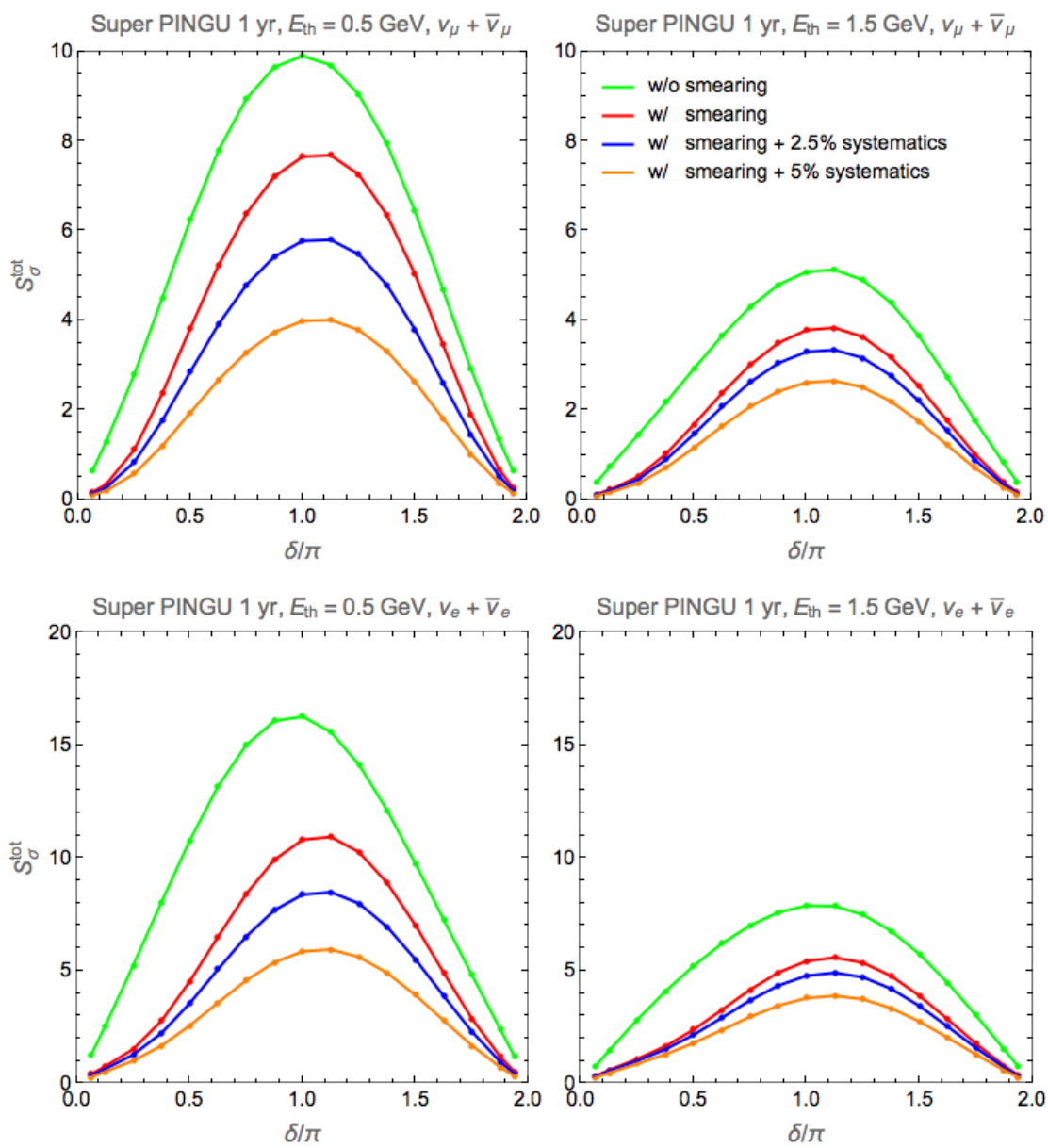

Figure 9. Integrated Super-PINGU distinguishabilities between a given value of $\delta$ and $\delta=0$ as functions of $\delta$ for the $\nu_{\mu}+\bar{\nu}_{\mu}$ events (upper panels) and for $\nu_{e}+\bar{\nu}_{e}$ events (lower panels). The dependencies have been computed for the energy thresholds $E_{\mathrm{th}}=0.5 \mathrm{GeV}$ (left panels) and $E_{\text {th }}=1.5 \mathrm{GeV}$ (right panels). Different lines show distinguishabilities without smearing, with smearing and different levels of the uncorrelated systematic errors: $f=0,2.5 \%$ and $5 \%$. Normal mass hierarchy is assumed.

Smearing suppresses $S_{\sigma}\left(\nu_{e}\right)$ stronger than $S_{\sigma}\left(\nu_{\mu}\right)$, especially in the interval $\delta=(0-$ $0.5) \pi$. For $E_{\mathrm{th}}=0.5 \mathrm{GeV}$ the factors of suppression are (1.4-3.5) for $\nu_{e}$ and (1.3-2.3) for $\nu_{\mu}$. Here in brackets, small number corresponds to $\delta=3 \pi / 2$ and large one to $\pi / 4$. For $\delta=(0.5-1.5) \pi$ the suppression is given by 1.3 for $\nu_{\mu}$ and (1.4-1.5) for $\nu_{e}$.

(ii) The systematic uncorrelated errors suppress $S_{\sigma}$ but do not affect significantly the shape of $S_{\sigma}$ dependence on $\delta$. For $E_{\text {th }}=0.5 \mathrm{GeV}$, the $f=2.5 \%$ level errors diminish $S_{\sigma}\left(\nu_{\mu}\right)$ by a factor $(1.3-1.5)$ in the interval $\delta=(0.25-1.5) \pi$. In this interval $S_{\sigma}\left(\nu_{e}\right)$ is suppressed by a factor $\approx 1.3$. An error $f=5 \%$ gives an additional suppression with respect to $f=2.5 \%$ case: e.g., for $\delta=\pi$ the suppression factor is 1.4 for both $S_{\sigma}\left(\nu_{\mu}\right)$ and $S_{\sigma}\left(\nu_{e}\right)$. With increase of $E_{\mathrm{th}}$, effect of the uncorrelated systematics decreases since the number of events decreases. For Super-PINGU with $E_{\mathrm{th}}=1.5 \mathrm{GeV}$ we 

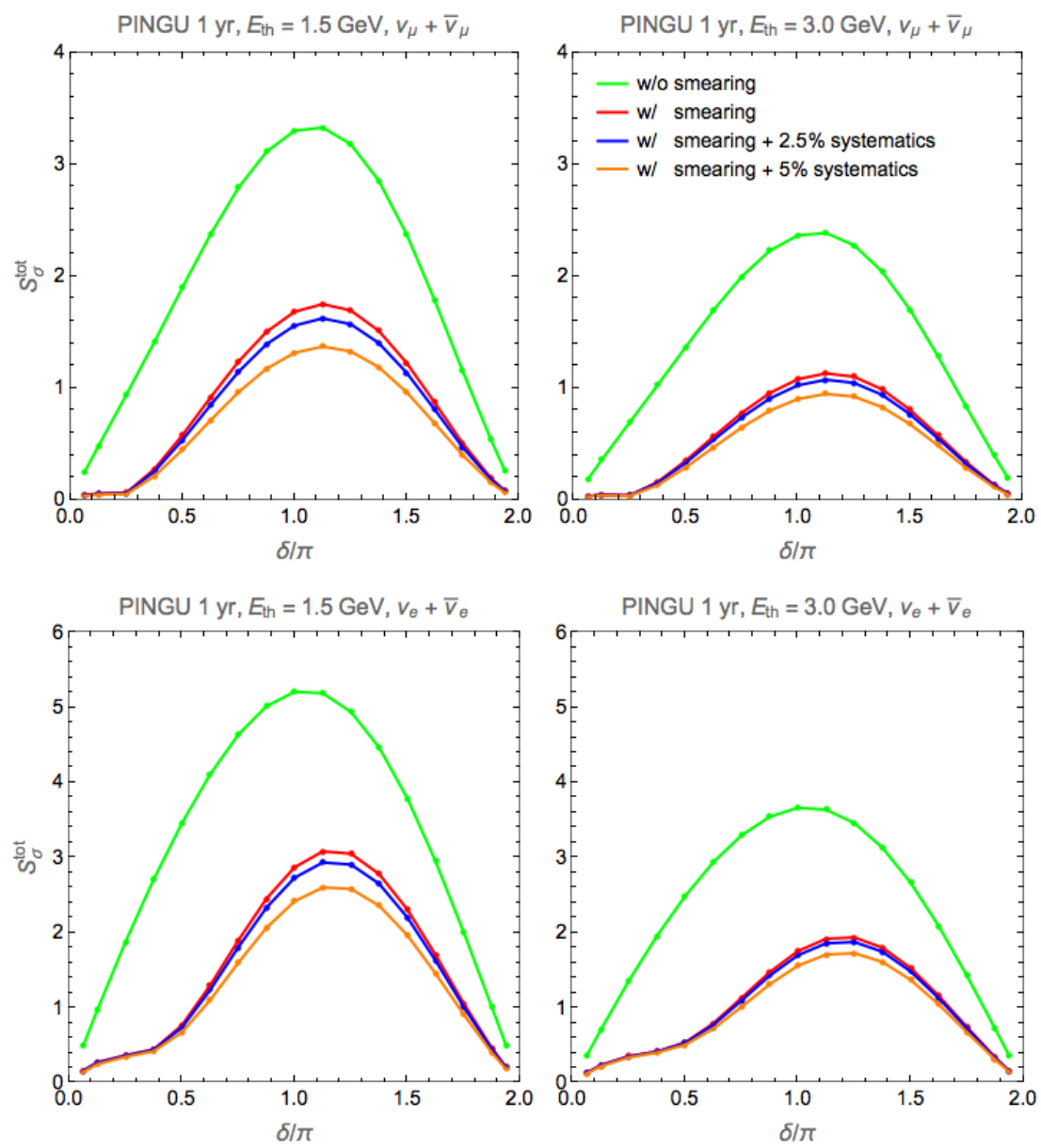

Figure 10. The same as in figure 9, but for the PINGU detector. The energy thresholds are 1.5 $\mathrm{GeV}$ and $3.0 \mathrm{GeV}$ in this case.

obtain about 2 times smaller suppressions for $\delta=\pi$ : $S_{\sigma}\left(\nu_{\mu}\right)$ decreases by a factor 1.15 (1.46) for $f=2.5 \%(5 \%)$ and $S_{\sigma}\left(\nu_{e}\right)$ decreases even weaker by a factor 1.13 (1.40). For PINGU with $E_{\mathrm{th}}=3 \mathrm{GeV}$ the reduction is $4 \%(12 \%)$ for $f=2.5 \%(5 \%)$.

(iii) With decrease of threshold from $1.5 \mathrm{GeV}$ down to $0.5 \mathrm{GeV}$, the integral distinguishability of Super-PINGU increases in the interval $\delta=(1.0-1.5) \pi$ by a factor $\sim 1.7$ for both types of events. For $\delta \leq \pi / 2$ the increase is bigger: (1.9-2.0) for $\nu_{\mu}$ and smaller: $(1.4-1.7)$ for $\nu_{e}$.

(iv) Smearing reduces distinguishability for PINGU much stronger than for super-PINGU (see figure 10). Moreover the suppression increases with decrease of $\delta$. Taking $E_{\mathrm{th}}=$ $1.5 \mathrm{GeV}$ we obtain the following numbers for suppression factor of $S_{\sigma}\left(\nu_{\mu}\right)\left(S_{\sigma}\left(\nu_{e}\right)\right)$ : $\delta=3 \pi / 2: 1.64(1.62), \delta=\pi: 2(1.9), \delta=\pi / 2: 3.2(4.5), \delta=\pi / 4:>20$ (4.5). Such dependence is related to bigger width of the reconstruction function and comparable regions of the positive and negative $S_{\sigma}$ before smearing. Similar factors arise for $E_{\text {th }}=3 \mathrm{GeV}$. In contrast, the uncorrelated systematic errors affect PINGU sensitivity 

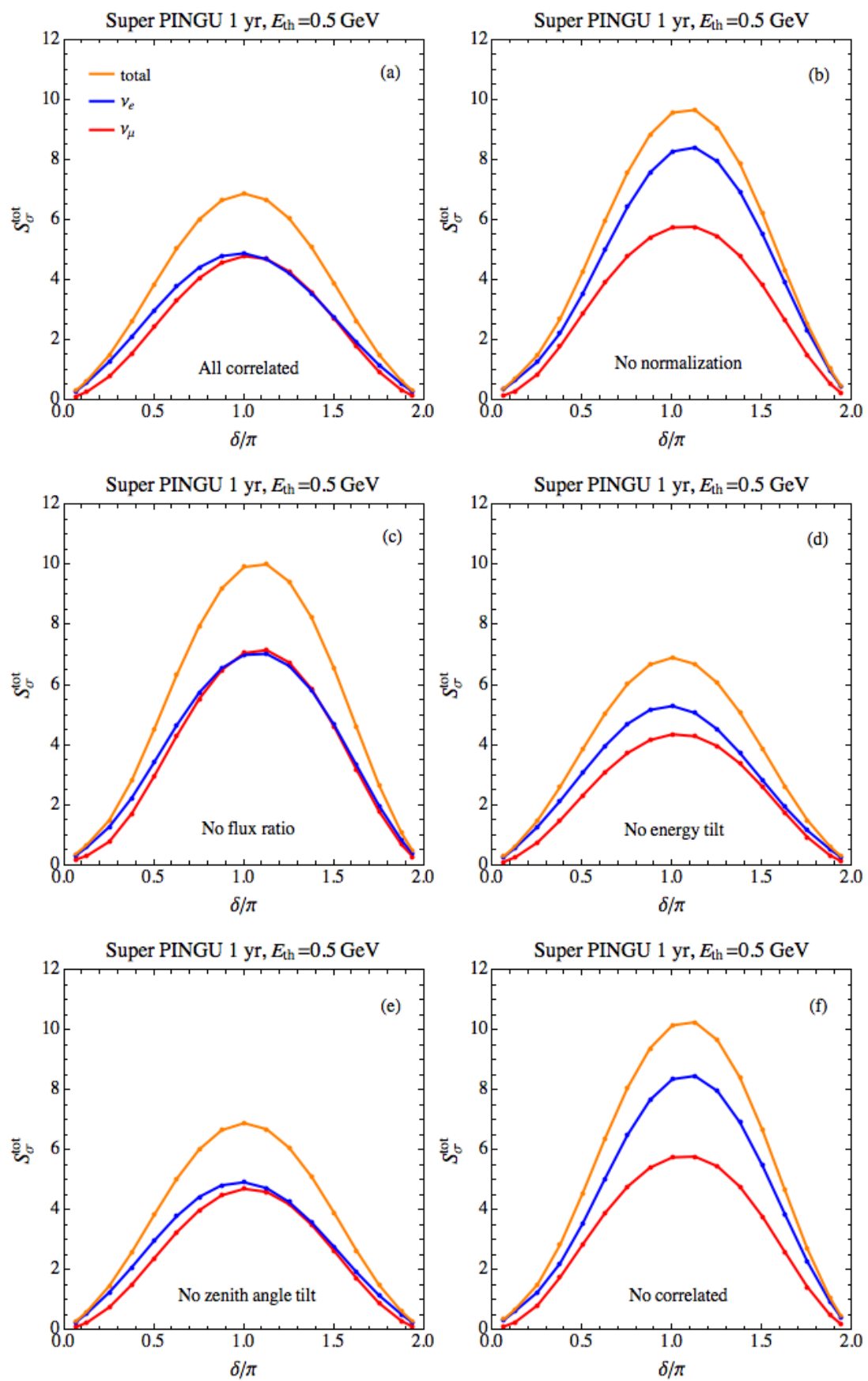

Figure 11. Effects of different correlated systematic errors on sensitivity to the CP-phase. Shown are the total distinguishability as well as integrated Super-PINGU distinguishabilities from $\nu_{\mu}$ and $\nu_{e}$ events between a given value of $\delta$ and $\delta=0$ as functions of $\delta$. Different panels correspond to the cases when (a) all errors are included; (b) normalization uncertainty of $20 \%$ is removed; (c) flux ratio uncertainty is removed; (d) the energy tilt uncertainty is removed; (e) the angular tilt uncertainty is removed; (f) all correlated systematic uncertainties are removed. The distinguishabilities have been computed after smearing, with $2.5 \%$ uncorrelated systematics 1 year exposure, $E_{\text {th }}=0.5 \mathrm{GeV}$ and for sum of $\nu$ and $\bar{\nu}$ signals. 
much weaker (less than 10\%) than Super-PINGU, which is related to smaller number of PINGU events.

Comparing PINGU and Super-PINGU at the same threshold, $E_{\text {th }}=1.5 \mathrm{GeV}$, we obtain that distinguishability for Super-PINGU is bigger by a factor (1.7-2.4) in the interval $\delta=(1-1.5) \pi$, mainly due to increase of the effective volume. For $\delta \leq \pi / 2$ the increase is much bigger: $>5$ due to both increase of $V_{\text {eff }}$ and better reconstruction.

Going from PINGU with $E_{\text {th }}=3 \mathrm{GeV}$ to Super-PINGU with $E_{\text {th }}=0.5 \mathrm{GeV}$ (with smearing and $2.5 \%$ systematics) the distinguishability increases by a factor (6-7) for $\nu_{\mu}$ and by a factor $(4-5)$ for $\nu_{e}$ in the interval $\delta=(1-1.5) \pi$.

(v) We have also computed the Super-PINGU distinguishability using the PINGU reconstruction functions without rescaling. Improvement of the reconstruction $(\sqrt{3}$ decrease of widths) affects very weakly the distinguishability in the range $\delta=(1-$ $1.5) \pi$. For $\delta=\pi / 2$ we find $25 \%$ increase of $S_{\sigma}\left(\nu_{e}\right)$ and $10 \%$ increase of $S_{\sigma}\left(\nu_{\mu}\right)$. The improvement is very strong for small values of $\delta$. Maximal increase of $S_{\sigma}\left(\nu_{e}\right)$ given by a factor 2 is at $\delta=\pi / 4$ and maximal increase of $S_{\sigma}\left(\nu_{\mu}\right)$ is by a factor 2.2 at $\delta=\pi / 8$. The improved resolution for large $\delta$ is important for measurements of $\delta$.

We define the total distinguishability (both $\nu_{\mu}$ and $\nu_{e}$ channels) as

$$
S_{\sigma}^{\text {tot }}=\sqrt{S_{\sigma}^{2}\left(\nu_{\mu}\right)+S_{\sigma}^{2}\left(\nu_{e}\right)}
$$

As the reference setup we take Super-PINGU with $0.5 \mathrm{GeV}$ threshold and $2.5 \%$ systematics. We use 4 years exposure and $\sqrt{t}$ scaling of the total distinguishability with exposure time. According to figure 9 and figure 11 (f), the phases $\delta=\pi / 4, \pi / 2, \pi, 3 \pi / 2$ can be distinguished from zero at $S_{\sigma}^{\text {tot }}=3.0,9,21$ and 13.4 correspondingly. The contribution from $\nu_{e}$ events is about (1.4-1.5) times larger than that from $\nu_{\mu}$ events alone. For $E_{\text {th }}=1.5 \mathrm{GeV}$, the total distinguishability $S_{\sigma}^{\text {tot }}=2.1,5.0,11.5$ and 7.9 for $\delta=\pi / 4, \pi / 2, \pi$, and $3 \pi / 2$ respectively. So, the decrease is by a factor $(1.5-1.7)$.

\subsection{Correlated systematic error}

The correlated systematic errors require special consideration in view of the facts that effects of CP-differences are small, $\sim(1-2) \%$, the $\left(E_{\nu}-\cos \theta_{z}\right)$ distributions are rather flat (especially in the region of relatively large CP-violation at low energies), and at low energies the asymmetry (after smearing) has the same sign for all zenith angles. The most important correlated systematic errors are related to uncertainties in

- the atmospheric neutrino fluxes. In the first approximation they can be described by a) the overall normalization factor, b) the energy spectrum tilt; c) the flux (flavor) ratio, and d) the zenith angle dependence tilt. At low energies the azimuthal dependence also becomes non-trivial.

- the cross-sections of neutrino and antineutrino interactions. Uncertainties should be considered separately for different reactions. 
- the effective volume $V_{\text {eff }}$ and its energy dependence.

- parameters of the reconstruction functions.

- neutrino mixing angles and mass squared differences.

The cross-sections, fluxes and effective volumes enter expressions for numbers of events as a product $\sigma \Phi V_{\text {eff }}$, therefore their uncertainties can be described simultaneously: so that the number of $l$-events in $i j$ bin with the uncertainties included equals

$$
N_{i j, l}^{\delta}\left(\delta, \xi_{k}\right)=\alpha z_{l}\left(\frac{E}{2 \mathrm{GeV}}\right)^{\eta}\left[1+\beta\left(0.5+\cos \theta_{z}\right)\right] N_{i j, l}^{\delta}\left(\xi_{k}^{s t}\right), \quad l=e, \mu .
$$

Here $\alpha$ is the overall normalization factor with error $\sigma_{\alpha}=0.2, z_{l}$ is the flux (flavor) ratio uncertainty ( $z_{e} \equiv 1$ for $\nu_{e}$ events) with error $\sigma_{z}=0.05, \eta$ is the energy tilt parameter with error $\sigma_{\eta}=0.1, \beta$ is the zenith angle tilt with error $\sigma_{\beta}=0.04$. We denote these parameters collectively as

$$
\xi_{k} \equiv\left(\alpha, \beta, \eta, z_{\mu}\right), \quad \xi_{k}^{s t} \equiv(1,0,0,1) .
$$

In the distinguishability approach these uncertainties can be accounted for by modifying $S_{\sigma}^{\text {tot }}$ in the following way

$$
S_{\sigma}^{\mathrm{tot}}\left(\xi_{k}\right)=\sqrt{\sum_{l=e, \mu} \sum_{i j} \frac{\left[N_{i j, l}\left(\delta, \xi_{k}\right)-N_{i j}\left(\delta=0, \xi_{k}^{s t}\right)\right]^{2}}{\sigma_{i j, l}^{2}}+\sum_{k} \frac{\left(\xi_{k}-\xi_{k}^{s t}\right)^{2}}{\sigma_{k}^{2}}} .
$$

This modification is analogous to the pull method in $\chi^{2}$ analysis. $S_{\sigma}^{\text {tot }}\left(\xi_{k}\right)$ should be then minimized over all parameters $\xi_{k}$. Since $S_{\sigma}^{0}\left(\xi_{k}^{s t}\right)=0$ (there is no fluctuation in our analysis), $\min \left[S_{\sigma}\left(\xi_{k}\right)^{\text {tot }}\right]$ gives the final significance with systematic errors taken into account:

$$
S_{\sigma}^{\text {tot }}=\min \left[S_{\sigma}\left(\xi_{k}\right)^{\text {tot }}\right] .
$$

Notice that at minimization of $S_{\sigma}^{\text {tot }}$, the errors change the CP-differences in the individual $E_{\nu}-\theta_{z}$ bins, $S_{i j}$, in such a way that positive and negative $S_{i j}$ equilibrate, thus making them smaller in absolute value. This leads to decrease of total distinguishability, especially in the regions where $S_{i j}$ have the same sign.

Effects of different correlated errors on the total distinguishability are shown in figure 11. We present also the integrated distinguishabilities from the $\nu_{e}$ and $\nu_{\mu}$ events separately without the pull terms (last term of (6.4)). Figure 11(a) shows the distinguishabilities when all correlated errors are included. In the panels (b)-(e) we show the effects of removal of individual errors. In the panel (f) all correlated systematic errors are removed. Let us consider effects of different systematic errors in order

(i) The overall normalization of the product $\sigma_{\nu} \Phi_{\nu} V_{\text {eff }}$ modifies the CP-difference as

$$
S_{i j, l}=\frac{z_{l} \alpha N_{i j, l}(\delta)-N_{l, i j}(\delta=0)}{\sigma_{\beta}}=\frac{\left(z_{l} \alpha-1\right) N_{\mu}^{\text {ind }}+z_{l} \alpha N_{\mu}^{\delta}-N_{\mu}^{0}}{\sqrt{N_{\mu}^{\text {ind }}+N_{\mu}^{0}+f^{2}\left(N_{\mu}^{\text {ind }}+N_{\mu}^{0}\right)^{2}}}
$$


(recall that $\left.z_{e} \equiv 1\right)$. Here in the lowest (zero) approximation in $s_{13}$,

$$
N_{\mu}^{\text {ind }} \approx\left(s_{23}^{4}+c_{23}^{4}\right), \quad N_{e}^{\text {ind }} \approx \frac{1}{r} .
$$

(They correspond to the averaged $\nu_{\mu}-\nu_{\tau}$ oscillations.)

There are various factors which restrict the renormalization effect. Although in the case of independent analysis of the $\nu_{e}$ and $\nu_{\mu}$ events $S_{\sigma}\left(\nu_{\mu}\right)$ and $S_{\sigma}\left(\nu_{e}\right)$ can be strongly affected by the normalization uncertainty $\alpha$, the joint analysis of the $\nu_{\mu}$ and $\nu_{e}$ events shows only moderate reduction of $S_{\sigma}^{\text {tot }}$ (see figure 11). Indeed, according to (6.7) $N_{\mu}^{\text {ind }} \approx N_{e}^{\text {ind }} \approx 1 / 2$, i.e., they have the same sign and size. At the same time, as we have established before, the CP-differences of the $\nu_{e}$ events and the $\nu_{\mu}$ events have opposite signs. Consequently, $\left(\alpha N_{e}^{\delta}-N_{e}^{0}\right)$ and $\left(z_{\mu} \alpha N_{\mu}^{\delta}-N_{\mu}^{0}\right)$ in nominator of (6.6) have opposite signs. Also as we have seen the absolute values of these differences (after smearing) are of the same size. Therefore the overall normalization (the terms $\left.(\alpha-1) N_{e}^{\text {ind }}\right)$ can suppress $S\left(\nu_{e}\right)$ but then the term $\left(z_{\mu} \alpha-1\right) N_{\mu}^{\text {ind }}$ will enhance $S\left(\nu_{\mu}\right)$, or vice versa. According to figure 11 (b), removal of the normalization uncertainty increases $S\left(\nu_{\mu}\right)$ by a factor 1.14, and $S\left(\nu_{e}\right)$ by 1.66, whereas the total $S^{\text {tot }}$ increases by a factor 1.4. We find that reducing the normalization error down to $\sigma_{\alpha}=0.1$ practically does not change the result.

(ii) Essentially the freedom of normalization is restricted by the errors in the flavor ratio of fluxes, the ratio of cross sections and ratio of the effective volumes for $\nu_{e}$ and $\nu_{\mu}$. Indeed, according to figure 11 switching off the uncertainty in the flux ratio increases both $S_{\sigma}\left(\nu_{\mu}\right)$ and $S_{\sigma}\left(\nu_{e}\right)$ by a factor $\approx 1.36$ and $S_{\sigma}^{\text {tot }}$ by 1.4 , as in the case of normalization.

Other factors which restrict the normalization uncertainty effect (also other tilts uncertainties) include the following

- In the "core domain" ( $\left.\cos \theta_{z}<-0.83\right)$ the sign of the CP difference is opposite to that in the "mantle domain" $\left(\cos \theta_{z}>-0.83\right)$. So, a suppression of the sensitivity to $\delta$ in mantle enhances sensitivity in the core domain.

- Since at high energies, effect of CP phase becomes negligible the overall shift can not be significant.

- For the down-going events (although at low energies it is difficult to determine the direction) the $\mathrm{CP}$ and oscillation effects are small. Therefore inclusion of these events will further restrict the uncertainty of normalization. In other words, one can use the down-going neutrino events to fix the overall normalization.

- The CP differences for neutrinos and antineutrinos have opposite signs, and the overall normalization factor can diminish only one difference.

(iii) The energy tilt uncertainty in the form (6.2), see figure 11 (d), produces only (2-3)\% decrease of sensitivity. 
(iv) Exclusion of the angular tilt uncertainty, as follows from figure 11 (e), leads to few percent increase of $S_{\sigma}^{\text {tot }}$. It is not excluded, however, that the tilt uncertainties in some other form will lead to stronger diminishing. However, this is partially accounted for by the uncorrelated systematic errors.

(v) Removal of all correlated uncertainties produces similar effect on $S_{\sigma}^{\text {tot }}$ as removal of the normalization or the flux ratio uncertainties see, figure 11 (f).

The energy scale uncertainty, $E \rightarrow E+\epsilon$, gives $N^{\delta} \rightarrow N^{\delta}(1+\epsilon / E)^{-\gamma+1}$, where $\gamma$ is the spectral index of the atmospheric neutrinos. In the case of linear uncertainty, $\epsilon \propto E_{\nu}$, the effect is reduced to renormalization. Here we can extrapolate result on the absolute energy scale uncertainty from the PINGU simulation [34], which is small.

Uncertainties of the reconstruction functions (width, shape) can be estimated after the corresponding simulations will be done in future. We expect that by the time of operation of Super-PINGU, the values of neutrino oscillation parameters will be measured with high enough accuracy and we do not include their errors in the present estimations.

For simplicity we fixed all the oscillation parameters to their present best fit values, assuming that they will be known well enough by the time of Super-PINGU measurements. Notice that the 2-3 mixing is degenerate with the CP-violating phase, especially for the beam experiments (see for example [49]). In contrast to the beam experiments, there is no substantial degeneracy of the CP phase and the 2-3 mixing in the atmospheric neutrino experiments. The key point is that with atmospheric neutrinos one measures two dimensional distributions in wide range of energy and wide range of baseline (zenith angle). Furthermore, both appearance and disappearance channels contribute. Change of pattern of distribution with value of the 2-3 mixing and $\delta$ are different. In particular, the highest sensitivity to the 2-3 mixing is at high energies and large $\left|\cos \theta_{z}\right|$, as can be seen from figure 8 of [24] and figure 12. In figure 12 we show the distribution of the relative CP-differences for fixed $\delta=\pi$ and different values of $\theta_{23}$. According to figure 12 distribution of the $\nu_{\mu}$ events modifies substantially with $\theta_{23}$, which is due to the term $D_{23}$ in (4.2), whereas the distribution of the $\nu_{e}$ events practically does not change (see (5.7)). The highest sensitivity to $\delta$ is at low energies. Variations of $\theta_{23}$ can not mimic effect of the CP phase.

To further illustrate weak correlation between $\delta$ and $\theta_{23}$ in Super-PINGU we show in figure 13(a) dependence on $\sin ^{2} \theta_{23}$ of distinguishabilities $S_{e}, S_{\mu}$ and $S_{\text {tot }}$ between $\delta=0$ and $\delta=3 \pi / 2$. As follows from the figure in the range $\sin ^{2} \theta_{23}=0.45-0.55$ which can be achieved by PINGU, $S_{e}$ slightly increases; $S_{\mu}$ decreases from 2.3 down to 2.0 , and the total distinguishability decreases from 3.7 to 3.6 . So, variations of $\theta_{23}$ in this range can reduce the distinguishability by about $3 \%$. This shows that there is no significant degeneracy of $\theta_{23}$ and $\delta$.

We consider also the distinguishability between the distribution of events for phase $\delta=0$ and fixed 2-3 mixing $\sin ^{2} \theta_{23}=0.40, N(0,0.40)$, and the distributions of events 

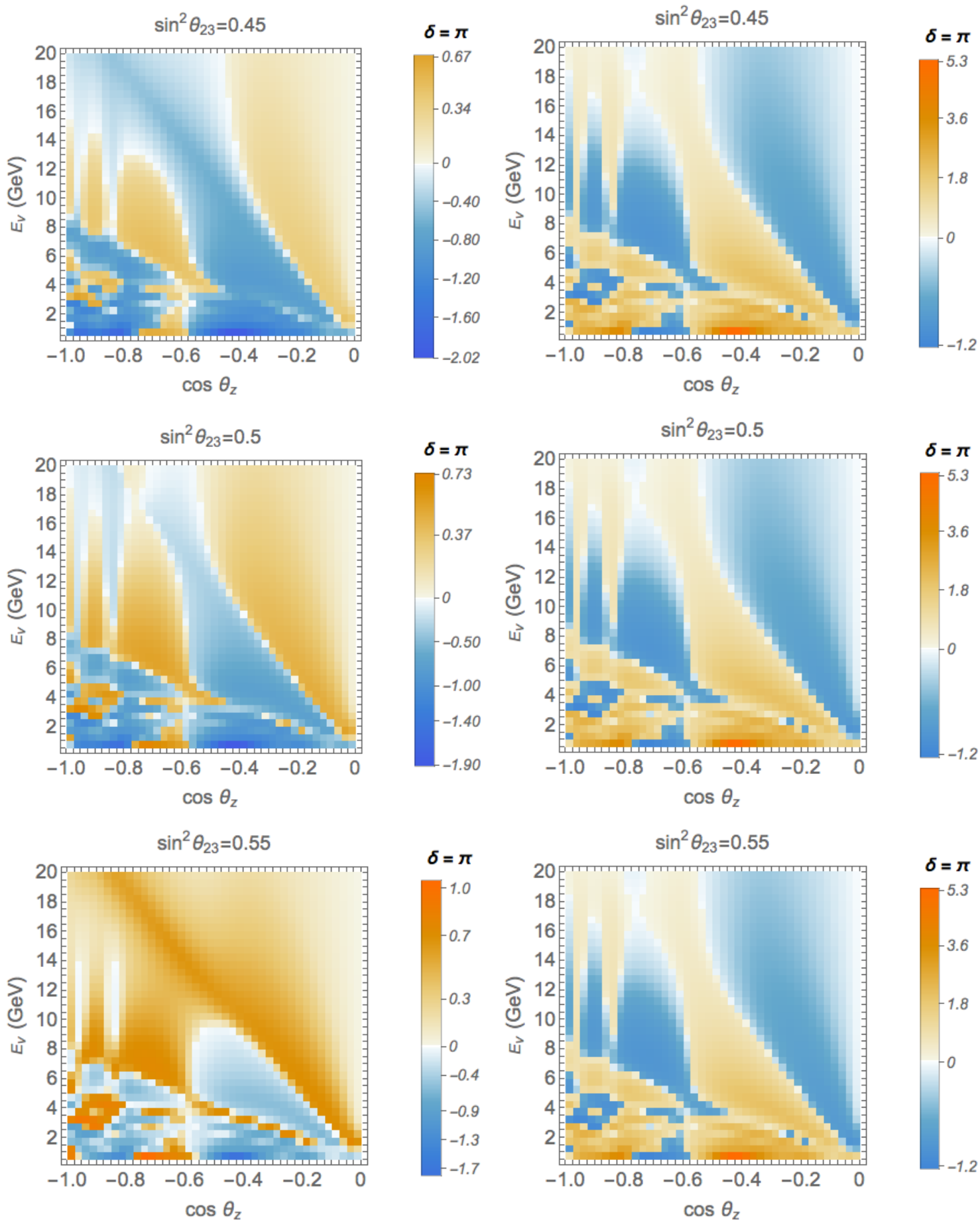

Figure 12. The distributions of the relative CP-differences for $\delta=\pi$ and different values of the 2-3 mixing. Left column for $\nu_{\mu}$ events, right column for $\nu_{e}$ events. 

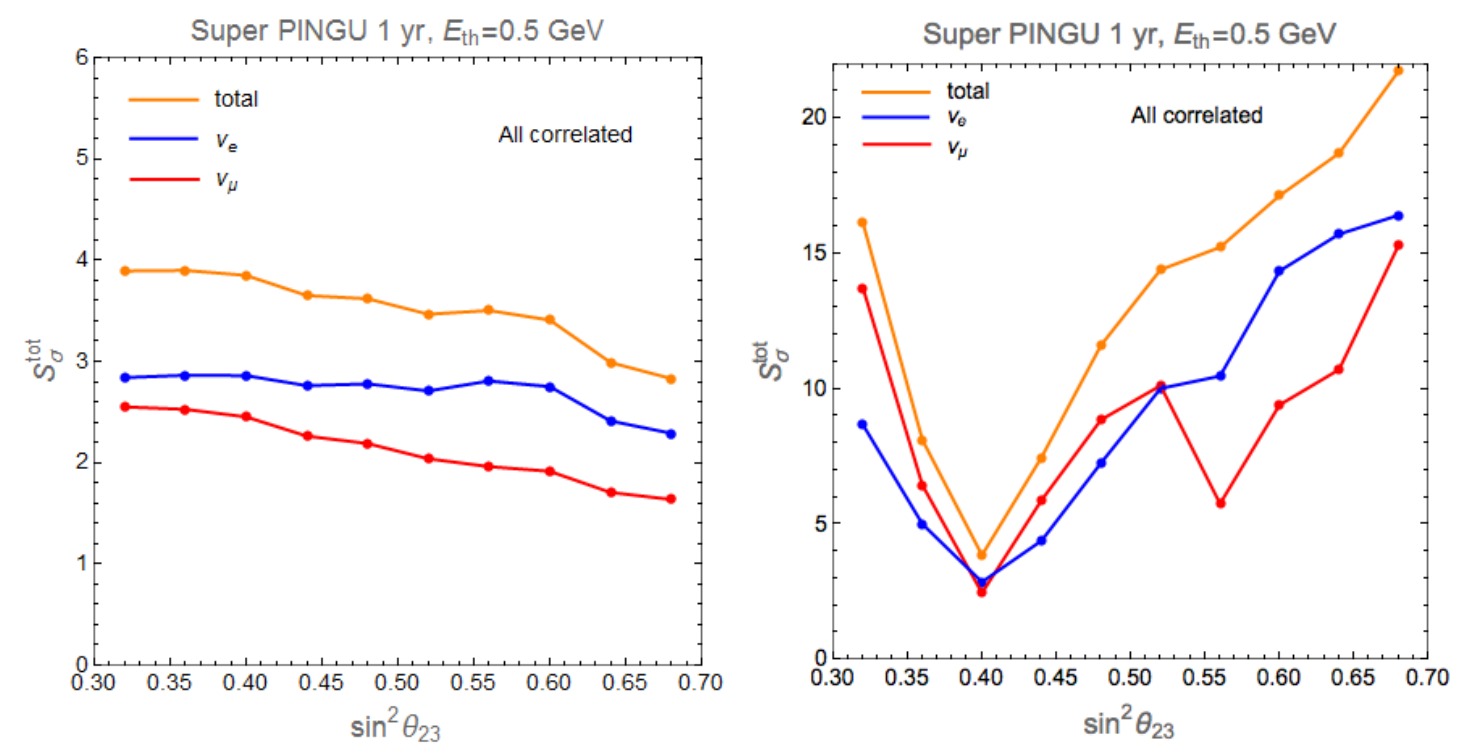

Figure 13. Dependence of distinguishabilities $S_{\sigma}\left(\nu_{e}\right)$ (blue), $S_{\sigma}\left(\nu_{\mu}\right)$ (red) and $S_{\sigma}^{\text {tot }}$ (orange) between $\delta=0$ and $\delta=3 \pi / 2$ on $\sin ^{2} \theta_{23}$. The distinguishabilities have been computed for superPINGU with $E_{\mathrm{th}}=0.5 \mathrm{GeV}$ and 1 year exposure. Panel (a): the same values of $\sin ^{2} \theta_{23}$ are taken for both distributions for both values of the phase; panel (b): the distribution for $\delta=0$ is taken for fixed value $\sin ^{2} \theta_{23}=0.40$.

for value of phase $3 \pi / 2$ and various values of $\sin ^{2} \theta_{23}, N\left(3 \pi / 2, \sin ^{2} \theta_{23}\right)$ :

$$
S_{\sigma}\left(\sin ^{2} \theta_{23}\right)=\frac{N(0,0.40)-N\left(3 \pi / 2, \sin ^{2} \theta_{23}\right)}{\sqrt{N(0,0.40)}} .
$$

In figure 13 (b) we show dependences of these $S_{\sigma}$ on $\sin ^{2} \theta_{23}$. According to this figure variations of $\sin ^{2} \theta_{23}$ do not change minimum of $S_{\sigma}$ which is at the same true value $\sin ^{2} \theta_{23}=0.40$ and equals 3.9. This means that variations of $\sin ^{2} \theta_{23}$ can compensate difference of phases. Furthermore, $S_{\sigma}$ increases fast with deviations of $\sin ^{2} \theta_{23}$ from the true value, which shows high sensitivity of superPINGU to 2-3 mixing. Thus, figure 13 (b) gives an idea on result of fit when both the phase and 2-3 mixing are varied simultaneously.

PINGU will be capable to improve determination of the 2-3 mixing substantially without knowledge of $\delta$. Both 2-3 mixing and $\delta$ can be determined from SuperPINGU. To minimize effects of $\theta_{23}$ one needs to analyze first the high energy data (say, above $10 \mathrm{GeV}$ ), where dependence on delta is very low (negligible), and analyze low energy data for sensitivity to $\delta$.

\subsection{Effect of flavor misidentification}

Let us consider first the $\nu_{\mu}$ (track) events. Due to misidentification they get contributions from $\nu_{e} \mathrm{CC}, \mathrm{NC}$ of all three neutrino types and $\nu_{\tau} \mathrm{CC}$ interactions. We will describe this by the misidentification parameters, $g_{\mu}^{\beta}$, which give fractions of events of type $\beta$ that are identified as the $\nu_{\mu}$ events. In turn, part of the true $\nu_{\mu}$ events will be identified as events 
of other types, e.g., the $\mathrm{NC}$ interactions. We will describe this by the fraction of $\nu_{\mu}$ events identified as tracks, $g_{\mu}^{\mu}$. Using these parameters the number of events identified as tracks (including misidentification) can be written as

$$
\tilde{N}_{\mu}(\delta)=g_{\mu}^{\mu}\left(N_{\mu}^{\mathrm{ind}}+N_{\mu}^{\delta}\right)+g_{\mu}^{e}\left(N_{e}^{\mathrm{ind}}+N_{e}^{\delta}\right)+g_{\mu}^{\mathrm{NC}} N_{\mathrm{NC}}+g_{\mu}^{\tau} N_{\tau}(\delta),
$$

where $N_{\beta}$ is the number of $\nu_{\beta}$ events without misidentification; recall that $N_{\mu}^{\text {ind }}+N_{\mu}^{\delta} \equiv$ $N_{\mu}(\delta)$, etc., and we have omitted the bin indices $i j$.

According to figure 9 of [34], the fractions of events $g_{\beta}^{\alpha}(\alpha \neq \beta)$ increase with decrease of energy reaching (for PINGU) $\approx 0.5$ at about $(1-2) \mathrm{GeV}$. In this case the suppression of the sensitivity to $\mathrm{CP}$ phase can be very strong. For super-PINGU misidentification is expected to be lower. For estimations we assume that $g_{\mu}^{\beta}$ and $1-g_{\mu}^{\mu}$ are $\sqrt{3}$ times smaller in Super-PINGU than in PINGU.

Although the fraction of the $\mathrm{CC} \nu_{\tau}$ events misidentified as tracks is large $(\sim 30 \%)$ (in PINGU), at energies below $3 \mathrm{GeV}$ the cross-section of the $\mathrm{CC} \nu_{\tau}$ interactions, and consequently $N_{\tau}$, is very small. So, we will neglect this contribution.

The NC events at low energies contribute to the track events when, e.g., charged $\pi$ is produced and misidentified with muon (CC $\nu_{\mu}$ events). These events have, however, smaller cross-section than the elastic scattering events. They contribute mostly in the region of $\Delta$ resonance.

For the CP-difference with misidentification taken into account we obtain

$$
\tilde{S}_{\mu}=\frac{g_{\mu}^{\mu}\left(N_{\mu}^{\delta}-N_{\mu}^{0}\right)+g_{\mu}^{e}\left(N_{e}^{\delta}-N_{e}^{0}\right)}{\sqrt{\tilde{N}(\delta=0)+[f \tilde{N}(\delta=0)]^{2}}},
$$

where according to $(6.9)$

$$
\tilde{N}(\delta=0) \approx g_{\mu}^{\mu} N_{\mu}(\delta=0)+g_{\mu}^{e} N_{e}(\delta=0)+g_{\mu}^{\mathrm{NC}} N_{\mathrm{NC}} .
$$

$\mathrm{NC}$ (as well as $N_{\mu}^{\text {ind }}$ ) do not contribute to nominator since $N_{\mathrm{NC}}$ does not depend on phase $\delta$ and there is no fluctuations in our approach.

As we discussed before, effects of change of $\delta$ have opposite signs for the $\nu_{e}$ and $\nu_{\mu}$ events, which leads to flavor suppression of the CP-differences. In general,

$$
N_{e}^{\delta}-N_{e}^{0}=-\zeta\left(N_{\mu}^{\delta}-N_{\mu}^{0}\right)
$$

where $\zeta=\zeta(r, \phi, \delta)=\zeta\left(E_{\nu}, \theta_{z}, \delta\right)>0$, see (4.2) and (5.2). Using this relation the expression for $\tilde{S}_{\mu}(6.10)$ can be written as

$$
\tilde{S}_{\mu}(f)=\kappa_{\mu} S_{\mu}\left(f^{\prime}\right)
$$

where $f^{\prime}=f \sqrt{g_{\mu}^{\mu}+R_{\mu}^{e} g_{\mu}^{e}+R_{\mu}^{\mathrm{NC}} g_{\mu}^{\mathrm{NC}}}$ is close to 1 and the suppression factor due to the misidentification equals

$$
\kappa_{\mu}=\frac{g_{\mu}^{\mu}-\zeta g_{\mu}^{e}}{\sqrt{g_{\mu}^{\mu}+R_{\mu}^{e} g_{\mu}^{e}+R_{\mu}^{\mathrm{NC}} g_{\mu}^{\mathrm{NC}}}} .
$$


Here

$$
R_{\mu}^{e} \equiv \frac{N_{e}(\delta=0)}{N_{\mu}(\delta=0)}, \quad R_{\mu}^{\mathrm{NC}} \equiv \frac{N_{\mathrm{NC}}}{N_{\mu}(\delta=0)}=\frac{\sigma_{\mathrm{NC}}}{\sigma_{\mathrm{CC}}} \frac{\Phi_{\mathrm{tot}}}{\Phi_{\mu}},
$$

and $\Phi_{\text {tot }}$ is the total neutrino flux at the detector which is not affected by oscillations. Taking in the first approximation, the $\delta$-independent parts of the probabilities we have according to (6.7) is $R_{\mu}^{e} \approx 2 r^{-1}$.

For estimations we take $\Phi_{\text {tot }}=\Phi_{\mu}^{0}\left(1+r^{-1}\right) \approx \Phi_{\mu}\left(c_{23}^{4}+s_{23}^{4}\right)^{-1}\left(1+r^{-1}\right) \approx 2 \Phi_{\mu}\left(1+r^{-1}\right)$. Here $\Phi_{\mu}^{0}$ is the muon flux at the production. Using $\sigma_{\mathrm{NC}} / \sigma_{\mathrm{CC}} \approx 1 / 3$ we obtain

$$
R_{\mu}^{\mathrm{NC}} \approx \frac{2}{3}\left(1+\frac{1}{r}\right)
$$

At low energies $(r=2), R_{\mu}^{\mathrm{NC}} \approx 1$ and with increase of energy it decreases down to $2 / 3$. In the first approximation we will also neglect difference between $f$ and $f^{\prime}$.

The minus sign in the nominator of $(6.13)(\zeta>0)$ is the main origin of suppression. For low energies, $E_{\nu}<2 \mathrm{GeV}$, when $r=2$ we have $\zeta=2$ independently of other parameters. For higher energies $\zeta$ can be found using the constant density approximation. From (4.6) and (5.7) we obtain after averaging out the second terms in both expressions:

$$
\zeta \approx \frac{r}{r-1} .
$$

With increase of $r$ (increase of energy) $\zeta \rightarrow 1$, and flavor suppression becomes weaker.

Similarly for $\nu_{e}$ events we can introduce the misidentification parameters $g_{e}^{e}$ and $g_{e}^{\beta}$. NC interactions contribute to $\tilde{N}_{e}$ via the $\pi^{0}$ production. The suppression factor for the CP-difference equals

$$
\kappa_{e} \approx \frac{g_{e}^{e}-\zeta^{-1} g_{e}^{\mu}}{\sqrt{g_{e}^{e}+\left(R_{\mu}^{e}\right)^{-1} g_{e}^{\mu}+R_{e}^{\mathrm{NC}} g_{e}^{\mathrm{NC}}}} .
$$

Here using similar consideration as for $\nu_{\mu}$ events we find

$$
R_{e}^{\mathrm{NC}} \equiv \frac{N^{\mathrm{NC}}}{N_{e}^{\mathrm{CC}}(\delta=0)} \approx \frac{1}{3}(r+1), \quad R_{e}^{\mu}=\left(R_{\mu}^{e}\right)^{-1} .
$$

At low energies, $R_{e}^{\mathrm{NC}} \approx 1$.

We have introduced the misidentification factors for individual $\left(E_{\nu}-\cos \theta_{z}\right)$ bins. This allows us to estimate suppression of distinguishability from certain energy regions. In particular, for low energies the factor $\zeta$ does not depend on the energy and angle, and if $g_{\beta}^{\alpha}$ are nearly constant we have for the integrated distinguishability: $\tilde{S}_{\text {int }}=\kappa S_{\text {int }}$. In general, one needs to take into account the energy and zenith angle dependence of $g_{\beta}^{\alpha}$ and perform integration with $\kappa_{e}$ and $\kappa_{\mu}$.

To perform estimations of the misidentification factors $\kappa_{\mu}$ and $\kappa_{e}$ we use the following relations between the misidentification parameters extracted from figure 9 of [34]:

$$
\left(1-g_{\mu}^{\mu}\right)=0.7 g_{\mu}^{e}, \quad g_{\mu}^{\mathrm{NC}} \approx 2 g_{\mu}^{e} .
$$

No results on $\nu_{e}$ misidentification is available, so we assume that $g_{e}^{\mu} \leq\left(1-g_{\mu}^{\mu}\right),\left(1-g_{e}^{e}\right) \geq g_{\mu}^{e}$, and $g_{e}^{\mathrm{NC}} \approx 2 g_{\mu}^{e}$. All the quantities here are expressed in terms of $g_{\mu}^{e}$ and for different values 
of this parameter we obtain, varying $\zeta$, the following: for $g_{\mu}^{e}=0.05: \kappa_{\mu}=(0.82-0.87)$ and $\kappa_{e}=(0.87-0.89)$; for $g_{\mu}^{e}=0.1: \kappa_{\mu}=(0.66-0.75)$ and $\kappa_{e}=(0.75-0.80)$; for $g_{\mu}^{e}=0.2: \kappa_{\mu}=$ $(0.38-0.55)$ and $\kappa_{e}=(0.55-0.63)$; for $g_{\mu}^{e}=0.3: \kappa_{\mu}=(0.15-0.38)$ and $\kappa_{e}=(0.38-0.46)$.

The distinguishability of the $\nu_{\mu}$ events is reduced more strongly than that for the $\nu_{e}$ events. According to figure 9 of [34], $g_{\mu}^{e}=0.1$ can be achieved by PINGU for $E_{\nu}>$ $30 \mathrm{GeV}$. If the parameters for Super-PINGU are scaled by a factor $\sqrt{3}$ we obtain instead $E_{\nu}>14 \mathrm{GeV}$. For $g_{\mu}^{e}=0.2$ the corresponding energies are $12 \mathrm{GeV}$ (PINGU) and $5 \mathrm{GeV}$ (Super-PINGU) and for $g_{\mu}^{e}=0.3$ we have $7 \mathrm{GeV}$ and $1 \mathrm{GeV}$. At the same time, at low energies where quasi-elastic scattering dominates the flavor identification can be better. We conclude that flavor identification at low energies is crucial for measurements of CPphase with Super-PINGU.

A consistent way to treat misidentification is to include its effects in simulations or use $g_{\beta}^{\alpha}$ as nuisance parameters and to perform marginalization over them. Unfortunately, only few parameters $g_{\beta}^{\alpha}$ are known even at high energies and their accuracy (say $1 \sigma$ intervals) are not clear. In this circumstance (in view of absence of information about $g_{\beta}^{\alpha}$ ) the correct question to address is what should be the size of $g$ (level of misidentification) which would allow to make measurement of the CP-phase. We answered this question by estimating effect on sensitivity of different values of $g$. We find that the level of misidentification should not be higher than $20 \%$.

Not only values of $g_{\beta}^{\alpha}$ but also accuracy with which $g_{\beta}^{\alpha}$ will be known are important for determination of $\delta$. Indeed, the uncertainties of misidentification parameters, $\delta g_{\beta}^{\alpha}$, propagate to the uncertainties of suppression factors $\kappa_{\mu}$ and $\kappa_{e}$, and consequently, will further reduce the distinguishability $S_{\alpha}$. If the errors of different $g_{\beta}^{\alpha}$ are uncorrelated the uncertainty in $\kappa_{\beta}$ can be written as

$$
\delta \kappa_{\beta}=\sqrt{\sum_{i}\left(\frac{d \kappa_{\beta}}{d g_{\beta}^{i}}\right)^{2}\left(\delta g_{\beta}^{i}\right)^{2}}, \beta=e, \mu, \quad i=e, \mu, N C .
$$

According to figure 9 of [34], in wide energy range the errors are rather small: $\delta g_{\beta}^{i}=0.01-$ 0.03 and they are about the same size for all the parameters: $g_{\beta}^{\alpha} \approx \delta g$. We assume that similar uncertainties will be for Super-PINGU. Then using expression (6.13) we obtain from (6.17) $\delta \kappa_{\mu} \approx 2.2 \delta g$ and $1.7 \delta g$ for $g_{\mu}^{e}=0.1$ and 0.2 correspondingly. The uncertainty in $\kappa_{e}$ is smaller: according to (6.16) and (6.17), $\delta \kappa_{e} \approx \delta g$ for both values of $g_{\mu}^{e}$. Taking $\delta g=$ 0.02 we have for $g_{\mu}^{e}=0.1$ that $\kappa_{\mu}=0.66 \pm 0.04$ and $\kappa_{e}=0.80 \pm 0.02$. The corresponding numbers for $g_{\mu}^{e}=0.2$ are $\kappa_{\mu}=0.380 \pm 0.035$ and $\kappa_{e}=0.63 \pm 0.02$. Consequently, uncertainty in $g_{\beta}^{\alpha}$ can reduce $S_{\mu}$ events by $(7-10) \%$ and $S_{e}-$ by $\sim 3 \%$, if $\delta g=0.02$.

\subsection{Sensitivity to CP-phase and its possible improvements}

Let us first summarize estimations of the total distinguishability of Super-PINGU in the case of 4 years of exposure, with $E_{\text {th }}=0.5 \mathrm{GeV}$, uncorrelated systematic errors at the level $f=2.5 \%$ and all correlated errors included. We have obtained that the values of phases $\delta=\pi / 4, \pi / 2, \pi, 3 \pi / 2$ can be distinguished from $\delta=0$ with $S_{\sigma}^{\text {tot }}=3.0,7.6,13.6,7.6$. Taking that the flavor misidentification averaged over energies reduces the distinguishability 
by a factor 1.3 , we have $S_{\sigma}^{\text {tot }}=2.1,5.3,9.5,5.3$ for the same values of the phase. That is, in the interval $\delta=(0.5-1.5) \pi$ the phase $\delta$ can be distinguished from 0 with $S_{\delta}^{\text {tot }}>5$. For $E_{\mathrm{th}}=1.5 \mathrm{GeV}$ we would have about a factor 1.7 lower distinguishability: $S^{\text {tot }}=$ $1.2,3.1,5.6,3.1$, so that in the interval $\delta=(0.5-1.5) \pi$ the phase can be distinguished from 0 at $S_{\sigma}^{\text {tot }}>3$. Lower level of the uncorrelated systematic error can increase $S_{\sigma}^{\text {tot }}$ by a factor 1.3 .

According to figures 9 and 10 correlated and uncorrelated systematic errors (even without misidentification) reduce significance by a factor of 1.5-2. Misidentification can further reduce it by a factor $0.5-0.7$. So, total effect of systematics is reduction of distinguishability by a factor of $2-4$. Therefore, systematics dominate in Super-PINGU as in beam experiments.

There are several possibilities to improve the sensitivity.

- Further decrease of the energy threshold. The increase of $S_{\sigma}^{\text {tot }}$ could be about $30 \%$ for $E_{\mathrm{th}}=0.2 \mathrm{GeV}$ in comparison with the case of $E_{\mathrm{th}}=0.5$. This would probably require a denser instrumentation of the detector.

- Stringent kinematical cuts: selection of subset of events with high quality of reconstruction of neutrino flavor, energy and direction. That will lead, however, to reduction of numbers of events (efficiency). In any case an optimization of quality of reconstruction and statistics is needed.

- Increase of the exposure time or/and increase of the effective volume. E.g., after 9 years the distinguishability for $\delta=\pi / 4$ can reach $S_{\sigma}^{\text {tot }} \approx 2-3$.

- Improvements of the flavor identification at low energies.

- Increase the photocathode area using denser array of DOMs or photosensors of new type, e.g., as considered for MICA. This will improve reconstruction of energy and direction of neutrino as well as flavor identification of events.

- Partial (statistical) separation of the neutrino and antineutrino signals can enhance distinguishability by up to $30 \%$.

We have computed the distinguishability from different values $\delta_{0}$ (apart from $\delta_{0}=0$ ). For $\delta_{0}=\pi$ the distinguishability can be obtained from results for $\delta_{0}=0$, see figure 14(a). In the first approximation the dependence is simply inverted, i.e., substituting maxima by minima and vice versa in figures 9-11. Equivalently, it is just shifted by about $\pi$. Situation is more complicated for other values of $\delta_{0}$ due to non-linear dependence of results on the phase. For distinguishability of $\delta$ from $\delta_{0}=3 \pi / 2$ (favored now) we find according to figure 14 (b) that for $\delta=\pi$ the distiguishability equals $S_{\sigma}^{\text {tot }}=3.5$. This is comparable with distinguishability between $3 \pi / 2$ and 0 discussed before. The largest $S_{\sigma}^{\text {tot }}=3.9$ is for $\delta=0$ and there is local minimum, $S_{\sigma}^{\text {tot }}=3$, for $\delta=\pi / 2$.

Our results show that Super-PINGU may be potentially competitive with other proposals [10-14]. Notice that accelerator experiments have good sensitivity at small values 

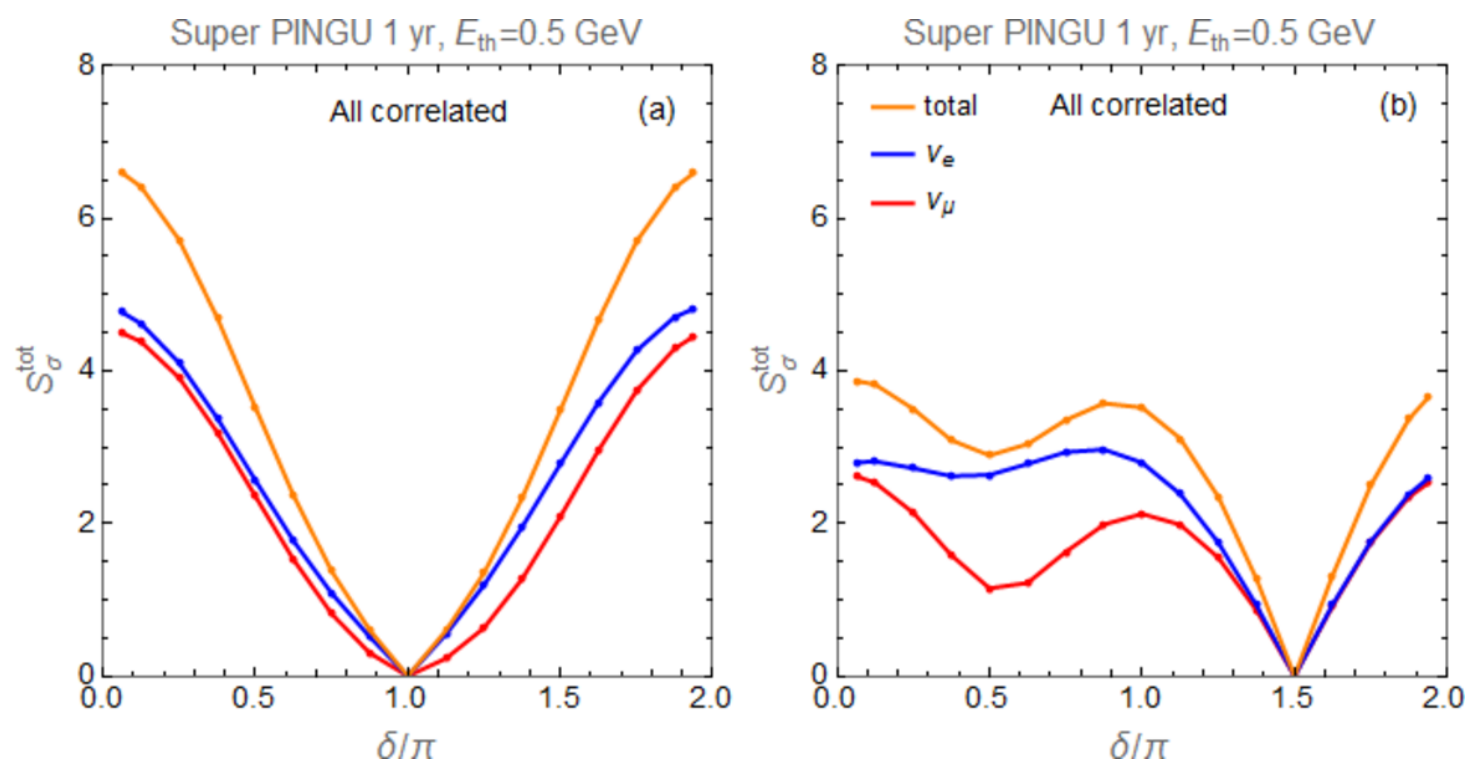

Figure 14. The distinguishabilities of a given value of $\delta$ from (a) $\delta_{0}=\pi$ and (b) $\delta_{0}=3 \pi / 2$. Shown are the total distinguishability (orange), as well as distinguishabilities from $\nu_{e}$ (blue) and $\nu_{\mu}$ (red) events as functions of the fit value of phase $\delta$.

of $\delta$ but typically show degeneracy at $\delta=0$ and $\pi \cdot{ }^{3}$ In contrast, Super-PINGU has relatively low sensitivity at $\delta<\pi / 2$, but distinguishability of $\delta=0$ and $\pi$ is nearly maximal. Interestingly, the strongest distinguishability is for $\delta=0$ and $\sim 3 \pi / 2$ (both for beams and Super-PINGU), consequently, if the present indications of $\delta \sim 1.5 \pi$ are true, it will be easier to establish the $\mathrm{CP}$-violation in lepton sector.

\subsection{Towards realistic estimation of sensitivity}

In view of the fact that characteristics of detection and reconstruction of neutrino parameters are largely unknown at low energies, we introduced a number of simplifications, assumptions and extrapolations of results from high energies. Therefore the emphasis is on identification of the main factors which affect the sensitivity rather than on final numbers, which should be considered as tentative and very preliminary. The crucial factors include:

- withds of the energy and angle reconstruction functions;

- flavor misidentification parameters;

- level of uncorrelated systematic errors.

Further progress can be achieved once PINGU (and ORCA) update their proposals and dedicated study of volume detection of the low energy events are performed.

We could have overestimated the sensitivity somehow, maybe by a factor of 2 but certainly not by an order of magnitude. This can be understood comparing our results

\footnotetext{
${ }^{3}$ This degeneracy is due to the fact that neutrino beam determinations of CP are based on measurements of the CP asymmetry (or $\nu-\bar{\nu}$ asymmetry) which is proportional to $\sin \delta$. Clearly, fit of all available data also sensitive to $\cos \delta$ will remove this degeneracy.
} 
with sensitivity of the HyperKamiokande atmospheric neutrino studies. According to [12] $\mathrm{HK}$ will be able to disentangle maximal $\mathrm{CP}$ violation from zero with $\sim 99 \%$ confidence level. These numbers are obtained after 10 years exposure and 0.56 Mton fiducial volume. Taking 10 times bigger effective volume we would get after 4 years with the same reconstruction capacities $5 \sigma$ confidence, which agrees with our estimations $(3-8 \sigma)$. Clearly Super-PINGU can not reach HyperKamiokande resolution but at the same time, since events we consider are at higher energies (0.5-1) $\mathrm{GeV}$ instead of $0.01 \mathrm{GeV}$, such a high quality instrumentation is not needed. Furthermore, volume detection may have advantages for higher energies. So, one would expect somehow lower than $5 \sigma$ significance.

Configuration of the Super-PINGU experiment is taken as an illustration. If needed, it is possible to consider denser array of photomultiplier tubes which will improve capacities of the detector. Furthermore, methods for volume detection of neutrinos are under fast development now. The progress is both in the directions of improvements of characteristics of the optical modules (DOM) and improvement of analysis of events (which differ from events in SK or HK). For instance, recent simulations by PINGU and ORCA show that reconstruction of the neutrino direction from the cascade events can be as good as from the tracking events.

Even if we have overestimated the sensitivity and real one is lower, this can be compensated by future developments of techniques and/or an increased density of DOMs in the detector. Results we present constitute a kind of reference point, which can be updated in different directions: one can improve quality of evaluation of sensitivity for a given configuration when more information will be obtained or one can change the assumed configuration of the detector.

Concerning the analysis, eventually distinguishability approach (which does not contain fluctuations) should be substituted by the $\chi^{2}$ or maximal likelihood analyses. For this, one needs to perform Monte Carlo (MC) simulations of events at Super-PINGU. This should be done by experimental collaborations and may take few years.

\section{Conclusions}

Assuming that the neutrino mass hierarchy is identified, we have explored a possibility to measure the CP-phase with future multi-megaton scale and low energy threshold atmospheric neutrino detectors. The method consists of comparison of the $\left(E_{\nu}-\cos \theta_{z}\right)$ distributions of events produced by $\nu_{e}$ and $\nu_{\mu}$ for different values of $\delta$. We use the relative CP-difference of the distributions to quantify distinguishability and sensitivity.

We have presented simple analytic formalism which allows us to describe properties of the distributions and gives their exact and explicit dependence on $\delta$. The pattern of distributions is determined to a large extent by the grid of magic lines of three types, which in turn determine the borders of the $\mathrm{CP}$ domains - the areas with the same sign of the CP-difference. At low energies the distributions are averaged over fast oscillations driven by the 1-3 mass splitting. Using the quasi-constant density approximation we have derived analytic expressions for the averaged distributions. In spite of this averaging the CP-effects are not washed out, and furthermore, increase with decrease of energy. 
In this connection we considered, as illustration, Super-PINGU which is a further possible upgrade of PINGU detector with multi-megaton effective volume in the sub-GeV range. Similarly, one can explore extensions of ORCA. Super-PINGU with large volume at $(0.1-0.2) \mathrm{GeV}$ can be used also for proton decay searches.

We have computed distributions of events and the relative CP-differences in the $\left(E_{\nu}-\right.$ $\left.\cos \theta_{z}\right)$ plane and studied their properties. There are various factors which suppress the observable CP-effects. In particular, the flavor suppression related to the presence of both $\nu_{\mu}$ and $\nu_{e}$ original fluxes, and the fact that CP-asymmetries of the $\nu_{\mu}-\nu_{\mu}$ and $\nu_{e}-\nu_{\mu}$ probabilities have opposite signs. This leads to partial cancellation of CP-phase effect in the $\nu_{e}$ and $\nu_{\mu}$ fluxes at the detector. The C-suppression is related to summation of the neutrino and antineutrino events, since $\nu$ and $\bar{\nu}$ have CP-asymmetries of opposite sign. This suppression could be reduced by partial separation of the $\nu$ and $\bar{\nu}$ signals.

Smearing of the distributions over the neutrino energy and direction reconstruction functions washes out fine structures of the distributions and leads to decrease of distinguishability by a factor (1.5-3), depending on the values of $\delta$. Distinguishability from $\delta=0$ is rather low for $\delta<\pi / 2$ and maximal in the interval $\delta=(1-1.5) \pi$.

Flavor misidentification of events at the detector produces strong decrease of sensitivity to the CP phase. Mainly, this is related to the fact that the $\nu_{e}$ and $\nu_{\mu}$ events have CPdifferences of opposite sign, and numbers of these events are comparable at low energies. Their misidentification leads to significant cancellation of the $\mathrm{CP}$ violation effects and suppression of $S_{\sigma}^{\text {tot }}$ can be by a factor $(4-5)$ at low energies. So, good flavor identification $\left(g_{\alpha}^{\beta}<0.2\right)$ is crucial for the CP phase measurement.

We find that inclusion of $f=2.5 \%$ uncorrelated errors (in the case of our binning and $\left.E_{\mathrm{th}}=0.5 \mathrm{GeV}\right)$ reduces the distinguishability by a factor (1.3-1.4). The correlated systematic errors can further reduce $S_{\sigma}^{\text {tot }}$ by about $30 \%-50 \%$. The total normalization of fluxes, and cross-sections uncertainties as well as uncertainties in the flux ratios give the main contribution to this reduction. Simultaneous analysis of $\nu_{\mu}$ and $\nu_{e}$ events allows to reduce effect of the correlated systematic errors.

Using figure 11(a) (line which corresponds to total distinguishability), we obtain that after 4 years of exposure (approximately double the numbers) and 2.5\% uncorrelated systematics the total distinguishabilities equal $S_{\sigma}^{\text {tot }}=3,8,14,8$ for the phases $\pi / 4, \pi / 2, \pi$, $3 \pi / 2$ correspondingly. As follows from our discussion in section 3.3, flavor misidentification at $20 \%$ level can reduce these numbers by a factor (0.3-0.5) (with stronger effect at smaller values of the phase). This will give minimal values: $S_{\sigma}^{\text {tot }}=1,3,6,3$ for the same phases in discussion. So, the value $\pi / 4$ can be distinguished from zero with $S_{\sigma}^{\text {tot }}=(1-3)$. For other phases we obtain $S_{\sigma}^{\text {tot }}(\pi / 2)=(3-8), S_{\sigma}^{\text {tot }}(\pi)=(6-14), S_{\sigma}^{\text {tot }}(3 \pi / 2)=(3-8)$. There are various reasons in addition which can modify these numbers (which in any case should be considered as tentative) in both directions. Conditionally $S_{\sigma}$ can be interpreted as significance and the corresponding quantities as numbers of sigmas. These estimations show that Super-PINGU may potentially be competitive with neutrino beam projects.

Going from PINGU with $E_{\mathrm{th}}=3 \mathrm{GeV}$ to Super-PINGU with $E_{\mathrm{th}}=0.5 \mathrm{GeV}$ increases the total distinguishability by a factor (4-6). The contributions of the $\nu_{\mu}$ and $\nu_{e}$ events to the total distinguishability can be comparable. 
Most of computations have been made assuming that by the time of Super-PINGU experiment the neutrino mass hierarchy will be established and for definiteness we took the normal mass hierarchy. We estimated that significance of measuring $\delta$ in the case of inverted mass hierarchy is about $30 \%$ lower.

The presented study of sensitivity to CP phase should be considered as very preliminary since various experimental features are not known yet. There is a number of issues related to detection of low energy events and determination of their characteristics (flavor, energy, direction, etc.). At the same time one can expect that new experimental developments will further improve the sensitivity. In any case, the results obtained here look very promising and encouraging and certainly show that "Super-PINGU for $\mathrm{CP}$ violation and proton decay" deserves further detailed study.

\section{Acknowledgments}

We are grateful to D. F. Cowen and J. P. A. Marcondes de André, E. Resconi and J. Koskinen for correspondence on the design and performance of the PINGU. S.R. thanks the Abdus Salam International Centre for Theoretical Physics for hospitality, where this work was initiated. S.R. acknowledges support from the National Research Foundation (South Africa) grant CPRR 2014 number 87823.

Open Access. This article is distributed under the terms of the Creative Commons Attribution License (CC-BY 4.0), which permits any use, distribution and reproduction in any medium, provided the original author(s) and source are credited.

\section{References}

[1] G.C. Branco, R.G. Felipe and F.R. Joaquim, Leptonic CP-violation, Rev. Mod. Phys. 84 (2012) 515 [arXiv:1111.5332] [INSPIRE].

[2] H. Nunokawa, S.J. Parke and J.W.F. Valle, CP violation and neutrino oscillations, Prog. Part. Nucl. Phys. 60 (2008) 338 [arXiv:0710.0554] [InSPIRE].

[3] P.A.N. Machado, H. Minakata, H. Nunokawa and R. Zukanovich Funchal, What can we learn about the lepton CP phase in the next 10 years?, JHEP 05 (2014) 109 [arXiv:1307.3248] [INSPIRE].

[4] T2K collaboration, P. de Perio, Oscillation results from T2K, arXiv:1405.3871 [INSPIRE].

[5] Super-Kamiokande collaboration, A. Himmel, Recent results from Super-Kamiokande, AIP Conf. Proc. 1604 (2014) 345 [arXiv: 1310.6677] [InSPIRE].

[6] F. Capozzi et al., Status of three-neutrino oscillation parameters, circa 2013, Phys. Rev. D 89 (2014) 093018 [arXiv: 1312.2878] [INSPIRE].

[7] D.V. Forero, M. Tortola and J.W.F. Valle, Neutrino oscillations refitted, Phys. Rev. D 90 (2014) 093006 [arXiv: 1405.7540] [INSPIRE].

[8] M.C. Gonzalez-Garcia, M. Maltoni and T. Schwetz, Updated fit to three neutrino mixing: status of leptonic CP-violation, JHEP 11 (2014) 052 [arXiv:1409.5439] [INSPIRE]. 
[9] M. Ghosh, P. Ghoshal, S. Goswami and S.K. Raut, Evidence for leptonic CP phase from

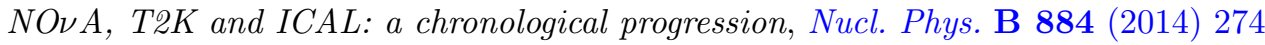
[arXiv: 1401.7243] [INSPIRE].

[10] LBNE collaboration, C. Adams et al., The long-baseline neutrino experiment: exploring fundamental symmetries of the universe, arXiv:1307.7335 [INSPIRE].

[11] V. Barger et al., Configurations of the long-baseline neutrino experiment, arXiv:1405.1054 [INSPIRE].

[12] K. Abe et al., Letter of intent: the Hyper-Kamiokande experiment - detector design and physics potential, arXiv:1109.3262 [INSPIRE].

[13] ESSnuSB collaboration, E. Baussan et al., A very intense neutrino super beam experiment for leptonic CP-violation discovery based on the European spallation source linac, Nucl. Phys. B 885 (2014) 127 [arXiv: 1309.7022] [INSPIRE].

[14] LAGUNA-LBNO collaboration, S.K. Agarwalla et al., The mass-hierarchy and CP-violation discovery reach of the LBNO long-baseline neutrino experiment, JHEP 05 (2014) 094 [arXiv: 1312.6520] [INSPIRE].

[15] M. Ghosh, P. Ghoshal, S. Goswami and S.K. Raut, Can atmospheric neutrino experiments provide the first hint of leptonic CP-violation?, Phys. Rev. D 89 (2014) 011301 [arXiv:1306.2500] [INSPIRE].

[16] O.L.G. Peres and A.Y. Smirnov, Atmospheric neutrinos: LMA oscillations, U(e3) induced interference and CP-violation, Nucl. Phys. B 680 (2004) 479 [hep-ph/0309312] [InSPIRE].

[17] K. Kimura, A. Takamura and H. Yokomakura, Enhancement of CP-violating terms for neutrino oscillation in earth matter, Phys. Lett. B 600 (2004) 91 [hep-ph/0407126] [INSPIRE].

[18] A. Takamura and K. Kimura, Large non-perturbative effects of small Delta $m_{21}^{2} / \Delta m_{31}^{2}$ and $\sin \theta_{13}$ on neutrino oscillation and CP-violation in matter, JHEP 01 (2006) 053 [hep-ph/0506112] [INSPIRE].

[19] K. Kimura, A. Takamura and T. Yoshikawa, Measuring the leptonic CP phase in $\nu_{\mu} \rightarrow \nu_{\mu}$ oscillations, Phys. Lett. B 642 (2006) 372 [INSPIRE].

[20] M.C. Gonzalez-Garcia and M. Maltoni, Atmospheric neutrino oscillations and new physics, Phys. Rev. D 70 (2004) 033010 [hep-ph/0404085] [INSPIRE].

[21] E.K. Akhmedov, M. Maltoni and A.Y. Smirnov, 1-3 leptonic mixing and the neutrino oscillograms of the Earth, JHEP 05 (2007) 077 [hep-ph/0612285] [INSPIRE].

[22] E.K. Akhmedov, M. Maltoni and A.Y. Smirnov, Neutrino oscillograms of the Earth: effects of 1-2 mixing and CP-violation, JHEP 06 (2008) 072 [arXiv:0804.1466] [INSPIRE].

[23] O. Mena, I. Mocioiu and S. Razzaque, Neutrino mass hierarchy extraction using atmospheric neutrinos in ice, Phys. Rev. D 78 (2008) 093003 [arXiv:0803.3044] [INSPIRE].

[24] E.K. Akhmedov, S. Razzaque and A.Y. Smirnov, Mass hierarchy, 2-3 mixing and CP-phase with Huge Atmospheric Neutrino Detectors, JHEP 02 (2013) 082 [arXiv:1205.7071] [INSPIRE].

[25] E.A. Hay and D.C. Latimer, Implications of the Dirac CP phase upon parametric resonance for sub-GeV neutrinos, Phys. Rev. C 86 (2012) 035501 [arXiv: 1207.5694] [inSPIRE]. 
[26] S.K. Agarwalla, T. Li, O. Mena and S. Palomares-Ruiz, Exploring the Earth matter effect with atmospheric neutrinos in ice, arXiv:1212.2238 [INSPIRE].

[27] T. Ohlsson, H. Zhang and S. Zhou, Probing the leptonic Dirac CP-violating phase in neutrino oscillation experiments, Phys. Rev. D 87 (2013) 053006 [arXiv: 1301.4333] [INSPIRE].

[28] S.-F. Ge, K. Hagiwara and C. Rott, A novel approach to study atmospheric neutrino oscillation, JHEP 06 (2014) 150 [arXiv:1309.3176] [INSPIRE].

[29] S.-F. Ge and K. Hagiwara, Physics reach of atmospheric neutrino measurements at PINGU, JHEP 09 (2014) 024 [arXiv: 1312.0457] [INSPIRE].

[30] V. Barger, D. Marfatia and K. Whisnant, Breaking eight fold degeneracies in neutrino CP-violation, mixing and mass hierarchy, Phys. Rev. D 65 (2002) 073023 [hep-ph/0112119] [INSPIRE].

[31] P. Huber and W. Winter, Neutrino factories and the 'magic' baseline, Phys. Rev. D 68 (2003) 037301 [hep-ph/0301257] [INSPIRE].

[32] A.Y. Smirnov, Neutrino oscillations: What is 'magic' about the magic baseline?, hep-ph/0610198 [INSPIRE].

[33] D.J. Koskinen, IceCube-DeepCore-PINGU: fundamental neutrino and dark matter physics at the South Pole, Mod. Phys. Lett. A 26 (2011) 2899 [inSPIRE].

[34] IceCube PINGU collaboration, M.G. Aartsen et al., Letter of intent: the Precision IceCube Next Generation Upgrade (PINGU), arXiv:1401.2046 [INSPIRE].

[35] D. Franco et al., Mass hierarchy discrimination with atmospheric neutrinos in large volume ice/water Cherenkov detectors, JHEP 04 (2013) 008 [arXiv: 1301.4332] [INSPIRE].

[36] W. Winter, Neutrino mass hierarchy determination with IceCube-PINGU, Phys. Rev. D 88 (2013) 013013 [arXiv: 1305.5539] [INSPIRE].

[37] K. Kimura, A. Takamura and H. Yokomakura, Exact formulas and simple CP dependence of neutrino oscillation probabilities in matter with constant density, Phys. Rev. D 66 (2002) 073005 [hep-ph/0205295] [INSPIRE].

[38] K. Kimura, A. Takamura and H. Yokomakura, Exact formula of probability and CP-violation for neutrino oscillations in matter, Phys. Lett. B 537 (2002) 86 [hep-ph/0203099] [InSPIRE].

[39] M. Blennow and T. Schwetz, Determination of the neutrino mass ordering by combining PINGU and Daya Bay II, JHEP 09 (2013) 089 [arXiv:1306.3988] [INSPIRE].

[40] A.M. Dziewonski and D.L. Anderson, Preliminary reference Earth model, Phys. Earth Planet. Interiors 25 (1981) 297.

[41] S. Böser, M. Kowalski, L. Schulte, N.L. Strotjohann and M. Voge, Detecting extra-galactic supernova neutrinos in the Antarctic ice, Astropart. Phys. 62 (2015) 54 [arXiv:1304.2553] [INSPIRE].

[42] D.F. Cowen, private communication.

[43] T. Arlen et al., Event reconstruction and particle identification for low energy events in DeepCore and PINGU, poster 368 at Neutrino 2014, $26^{\text {th }}$ International Conference on Neutrino physics and Astrophysics, June 2-7, Boston, U.S.A. (2014).

[44] D. Grant, Future atmospheric measurements with PINGU, talk given at Neutrino 2014, 26 ${ }^{\text {th }}$ International Conference on Neutrino physics and Astrophysics, June 2-7, Boston, U.S.A. (2014). 
[45] IceCube collaboration, A. Gross, Atmospheric neutrino oscillations in IceCube, Nucl. Phys. Proc. Suppl. 237-238 (2013) 272 [arXiv:1301.4339] [InSPIRE].

[46] M. Honda, T. Kajita, K. Kasahara and S. Midorikawa, Calculation of the flux of atmospheric neutrinos, Phys. Rev. D 52 (1995) 4985 [hep-ph/9503439] [INSPIRE].

[47] M. Sajjad Athar, M. Honda, T. Kajita, K. Kasahara and S. Midorikawa, Atmospheric neutrino flux at INO, South Pole and Pyhasalmi, Phys. Lett. B 718 (2013) 1375 [arXiv: 1210.5154] [INSPIRE].

[48] M. Ribordy and A.Y. Smirnov, Improving the neutrino mass hierarchy identification with inelasticity measurement in PINGU and ORCA, Phys. Rev. D 87 (2013) 113007 [arXiv: 1303.0758] [INSPIRE].

[49] P. Coloma, H. Minakata and S.J. Parke, Interplay between appearance and disappearance channels for precision measurements of $\theta_{23}$ and $\delta$, Phys. Rev. D 90 (2014) 093003 [arXiv: 1406.2551] [INSPIRE]. 